\title{
Catch bond models explain how force amplifies TCR signaling and antigen discrimination
}

\section{Authors}

Hyun-Kyu Choi ${ }^{1,2}$, Kaitao Li ${ }^{1,2}$, Muaz Nik Rushdi ${ }^{1,2, \dagger}$, Chenghao Ge ${ }^{1,2, \ddagger}$, Wei Chen ${ }^{3}$, Jizhong Lou $^{4-6}$, and Cheng $\mathrm{Zhu}^{1,2,7, *}$

\section{Affiliations}

\author{
${ }^{1}$ Wallace H. Coulter Department of Biomedical Engineering, Georgia Institute of \\ Technology and Emory University, Atlanta, Georgia 30332, USA \\ ${ }^{2}$ Parker H. Petit Institute for Bioengineering and Biosciences, Georgia Institute of \\ Technology, Atlanta, Georgia 30332, USA \\ ${ }^{3}$ Department of Cell Biology and Department of Cardiology of the Second Affiliated \\ Hospital, Zhejiang University School of Medicine, Hangzhou 310058, China \\ ${ }^{4}$ National Laboratory of Biomacromolecules, Institute of Biophysics, Chinese \\ Academy of Sciences, Beijing 100101, China \\ ${ }^{5}$ Key Laboratory of RNA Biology, CAS Center for Excellence in Biomacromolecules, \\ Institute of Biophysics, Chinese Academy of Sciences, Beijing 100101, China \\ ${ }^{6}$ University of the Chinese Academy of Sciences, Beijing 100049, China \\ ${ }^{7}$ George W. Woodruff School of Mechanical Engineering, Georgia Institute of \\ Technology, Atlanta, Georgia 30332, USA \\ *Corresponding Author: Cheng Zhu, Email: cheng.zhu@bme.gatech.edu \\ ${ }^{\dagger}$ Present address: Medtronic CO., Minneapolis, MN, USA \\ Present address: Amgen Inc., One Amgen Center Dr., Thousand Oaks, CA, USA
}

\begin{abstract}
Central to T cell biology, the TCR integrates forces in its triggering process upon interaction with pMHC. Phenotypically, forces elicit TCR catch-slip bonds with strong pMHCs but slip-only bonds with weak pMHCs. While such correlation is generally observed, the quantitative bond pattern and degree of "catchiness" vary. We developed two models based on the structure, elastic properties, and force-induced conformational changes of the TCR-pMHCI/II complexes to derive from their bond characteristics more intrinsic parameters that underlie
\end{abstract}


structural mechanisms, predict $\mathrm{T}$ cell signaling, and discriminate antigens. Applying the models to all published 48 datasets of 11 TCRs and their mutants interacting with corresponding pMHCs revealed the ability for structural and physical parameters to quantitatively integrate and classify a broad range of bond behaviors and biological activities. The extensive comparisons between theory and experiment allowed us to validate the models and identify specific conformational changes that control bond profiles, thereby providing structural insights into the inner workings of the TCR mechanosensing machinery and explaining why and how force amplifies TCR signaling and antigen discrimination.

\section{MAIN TEXT}

\section{Introduction}

Antigen recognition via interactions of the $\mathrm{T}$ cell receptor (TCR) with peptide-major histocompatibility complex (pMHC) is essential for $\mathrm{T}$ cell activation, differentiation, proliferation, and function (1). Mechanical forces applied to TCR via engaged pMHC substantially increase antigen sensitivity and amplify antigen discrimination (2-6). As a fundamental force-elicited characteristic, strong cognate pMHCs form catch-slip bonds with TCR where bond lifetimes increase with force until reaching a peak, and decrease as force increases further, whereas weak agonist and antagonist pMHCs form slip-only bonds with TCR where bond lifetimes decrease monotonically with increasing force $(2-5,7)$. However, the mechanism underlying the correlation between the force-lifetime pattern and the ability for force on TCR to induce T cell signaling remains unclear.

An intuitive hypothesis is that catch bonds prolong interactions, which allow the process of CD3 signal initiation to proceed sufficient number of phosphorylation steps to the threshold for downstream signal propagation, as proposed by the kinetic proofreading model (8). However, this hypothesis faces two challenges upon scrutiny of multiple TCR-pMHC 
systems. First, since bond lifetime vs force profiles are monotonically decreasing for slip-only bonds but bell-shaped for catch-slip bonds, the type of bonds that would last longer depends on the force range, which can switch from catch-slip bonds to slip-only bonds, and vice versa. Second, TCR-pMHC interactions exhibiting catch-slip bonds often have longest lifetimes around $10-20 \mathrm{pN}(2-5,7,9)$ and it has been reported that upon engaging $\mathrm{pMHC}$, T cells would exert 12-19 pN endogenous forces on the TCR in a signaling-dependent fashion (10). However, the relevance of this force range to $\mathrm{T}$ cell signaling remains incompletely understood. More perplexingly, some signal-inducing pMHC ligands form catch-slip bonds with TCRs but exhibit shorter lifetime than other pMHC ligands that do not induce signaling by, and form slip-only bonds with, the same TCRs even in the optimal force range (11). These observations prompt the questions of what mechanism underlies the association of TCR-pMHC bond type with the T cell signaling capacity and what impact do this specific force-range have on TCR mechanotransduction. To answer these questions requires an in-depth analysis of the multiple datasets with mathematical models, which was lacking.

Slip and catch bonds refer to two opposite effects of physical force on biomolecular interactions: increasing or decreasing their off-rate of dissociation, respectively $(12,13)$. Because force tends to be disruptive, slip bonds are intuitive, whereas catch bonds are counterintuitive. Since excessive force can rupture even covalent bonds (14), continued force increase will eventually overpower any catch bond, turning it to a slip bond after an "optimal" force where the off-rate is minimal $(2-5,7,13)$. Slip bond behavior is commonly modeled by the Bell equation, which assumes the dissociation rate $k$ of a molecular bond along a single pathway in a one-dimensional (1D), single-well energy landscape to be an exponential function of force, $k(F)=k_{0} e^{\frac{\delta_{0}^{*} F}{k_{\mathrm{B}} T}}$. Here, $k_{0}$ is the transition rate at zero force, $F$ is tensile force, $\delta_{0}^{*}$ is the forcefree distance from the bound state at the bottom of the energy well to the top of the energy 
barrier known as the "transition state", $k_{\mathrm{B}}$ is the Boltzmann constant, and $T$ absolute temperature (15). Several models have been developed to account for catch-slip bond behavior. Most introduced two dissociation pathways and/or two bound states in a two-dimensional (2D) energy landscape that is tilted by force (16). One noticeable exception is that of Guo et al. where dissociation is modeled to start from a single bound state along a single pathway through a 1D energy landscape based on the physical process of peeling a polymer strand with force until the transition state is reached (17). A distinct advantage of this model is its ability to relate the force-induced tilting of the energy landscape to the force-induced conformational change of the molecular complex (which all other models lack), thereby connecting parameters of the abstract energy landscape to the structural-elastic properties of the interacting molecules.

Besides binding properties of the TCR-pMHC complex, its structural features and conformational changes have been suggested to be important for TCR triggering. For example, TCR-pMHC docking orientation has been correlated to its ability to trigger $\mathrm{T}$ cell signaling $(11,18,19)$. Partial unfolding of either the TCR and/or MHC molecules has been inferred from mechanical experiments and steered molecular dynamics (SMD) simulations of pulling single TCR-pMHC bonds $(4,5)$. Whereas the extent of these conformational changes has been correlated to the strength of TCR-pMHC catch bonds $(4,5)$, the two phenomena have not been integrated into a mathematical model to explore their potential connection.

Here we developed two such models, one for each MHC class, to describe both TCR catch-slip and slip-only bonds. The model development follows Kramers' kinetic rate theory and uses polymer physics models to construct a 1D energy landscape for single-state, singlepathway dissociation that incorporates the structure, elastic properties, and force-induced conformational changes of the TCR-pMHC-I/II complexes at the sub-molecular level, which includes domain stretching, hinge rotation, and molecular extension. Applying the models to 
48 data sets of 11 TCRs and their mutants interacting with corresponding panels of both classes of pMHCs revealed the ability for structural and physical parameters to quantitatively integrate and classify a broad range of catch-slip or slip-only bond behaviors and biological activities. The extensive comparisons between theory and experiment allowed us to rigorously validate the models by systematically testing their assumptions, to identify specific conformational changes that control the bond profiles, to provide mechanistic insights into the inner workings of the TCR-pMHC mechanosensory machinery by constructing the energy landscape and investigating its properties, and to understand how force-elicited catch bond amplifies TCR signaling and antigen discrimination by examining the correlation of the model parameters with the biological activities of a large number of TCR-pMHC-I/II systems.

\section{Results}

\section{Model development}

Model goal: Kramers' kinetic rate theory treats bond dissociation as state transition in a 1D energy landscape $\Delta G^{*}\left(\delta_{\mathrm{l}}\right)$ from a free-energy well (bound state) over a barrier (transition state) along the dissociation coordinate $\delta_{1}(20)$. Following Guo et al. (17) to adapt the linearcubic model of Dudko et al. (21) but allow force $F$ to tilt the original energy landscape by an amount of $-\delta_{1} \gamma(F)$, the energy landscape takes the form of

$$
\Delta G\left(\delta_{\mathrm{l}}, F\right)=\frac{3 \Delta G_{0}^{*}}{2}\left(\frac{\delta_{1}}{\delta_{0}^{*}}-\frac{1}{2}\right)-2 \Delta G_{0}^{*}\left(\frac{\delta_{1}}{\delta_{0}^{*}}-\frac{1}{2}\right)^{3}-\delta_{1} \gamma(F) .
$$

where $\delta_{0}^{*}$ and $\Delta G_{0}^{*}$ are the differences in dissociation coordinates and free-energy levels, respectively, of the transition state and bound state of the original force-free energy landscape. The corresponding force-dependent kinetic rate is

$$
k(F)=k_{0} \sqrt{1-\frac{2 \delta_{0}^{*} \gamma(F)}{3 \Delta G_{0}^{*}}} \exp \left(\frac{\Delta G_{0}^{*}}{k_{\mathrm{B}} T}\left(1-\left(1-\frac{2 \delta_{0}^{*} \gamma(F)}{3 \Delta G_{0}^{*}}\right)^{3 / 2}\right)\right)
$$


where $k_{0}$ is the dissociation rate at zero force (17). Letting $\gamma \sim F$ recovers from Eq. 2 the Dudko-Hummer-Szabo model (21), and further assuming $\left|2 \delta_{0}^{*} \gamma(F) /\left(3 \Delta G_{0}^{*}\right)\right| \ll 1$ reduces it to the Bell model (15). The condition for $k$ to be able to model catch-slip bond is the derivative $k^{\prime}\left(F_{0}\right)=0$ where $F_{0}>0$. This translates to two conditions: the barrier height $\Delta G^{*}=$ $\Delta G_{0}^{*}\left(1-\frac{2 \delta_{0}^{*} \gamma\left(F_{0}\right)}{3 \Delta G_{0}^{*}}\right)^{3 / 2}=k_{B} T / 3$ or $\gamma^{\prime}\left(F_{0}\right)=0$. The first condition requires the energy change $-\delta_{1} \gamma$ induced by $F_{0}$ to lower the energy barrier height to $k_{\mathrm{B}} T / 2$ located at $\delta_{0}^{*}\left(\frac{k_{B} T}{2 \Delta G_{0}^{*}}\right)^{1 / 3}$. The second condition requires $\gamma$ to be a biphasic function of $F$. This excludes the Bell model and the Dudko-Hummer-Szabo model because both of their $\gamma$ functions depend on $F$ monotonically. Our goal is to construct a biphasic $\gamma(F)$ with appropriate structural-elastic dependency to account for TCR-pMHC conformational changes from the bound state to the transition state, as analyzed by the biomembrane force probe (BFP) experiments where a tensile force is applied to its two ends to modulate bond dissociation $(2,3,5,9)$.

Key model assumptions: Following the reasoning of Guo et al. (17), $\delta_{1} \gamma(F)=$ $\int_{0}^{F} \delta_{\mathrm{z}}(f) d f$ where the integrand $\delta_{\mathrm{z}}(f)=z(f)-z_{0}(f)$ is the projection on the force direction of the change induced by force $f$ of the TCR-pMHC extension at the transition state relative to its extension at the bound state. For $\gamma$ to depend on $F$ biphasically as required for describing catch-slip bonds, $\delta_{\mathrm{z}}$ should be a biphasic function of $f$ as discussed later. Therefore, dissociation occurs because the system moves in the energy landscape along the dissociation coordinate $\delta_{\mathrm{l}}$ from the bound state to the transition state by a distance $\delta^{*}=\delta_{0}^{*}\left(1-\frac{2 \delta_{0}^{*} \gamma(F)}{3 \Delta G_{0}^{*}}\right)^{1 / 2}$ (17). We assume that the differential contour length along the force transmission path across the TCR-pMHC structure (i.e., summing up all contour lengths of various domains connected at nodes of force action, as depicted in red lines in Fig. $1 A$, for MHC-I) at the transition state $l$ 
and bound state $l_{0}$ can serve as a dissociation coordinate, i.e., $\delta_{\mathrm{l}}=l-l_{0}$. $\delta_{\mathrm{z}}$ is the projection of $\delta_{\mathrm{l}}$ on the $\mathrm{z}$ axis - the direction of the pulling force (Fig. $1 A$ ). When only contour lengths are considered, $\delta_{\mathrm{l}}=\delta_{0}^{*}$, which serves as a criterion for finding the best-fit parameters (Fig. 1A, left and Supplemental Model Derivations, Eq. S7).

Suggested by single-molecule optical tweezers (4) and magnetic tweezers (5) experiments as well as steered MD (SMD) simulations (5), we assume that force-induced TCR-pMHC dissociation is accompanied by conformational changes in both the TCR and MHC molecules. Specifically, we assume that at the bound state, force induces elastic extension of the TCR-pMHC structure as a whole (Fig. 1A, left); but as the system moves toward the transition state for dissociation, conformational changes may occur, which may include disruption of intramolecular interfaces, hinge rotation, and interdomain joint partial unfolding (Fig. 1A, right). To include appropriate details of these proposed conformational changes at the sub-molecular level into the expression of $\delta_{\mathrm{z}}$, we model the TCR-pMHC structure as a system of semi-rigid bodies representing the whole complex as well as various globular domains connected by semi-flexible polymers that allow extension and hinge rotation under mechanical loads (Fig. 1A, right). Specifically, we assume that, as the system moves along the dissociation pathway from the bound state toward the transition state, force may induce disruption of the MHC $\alpha_{1} \alpha_{2}-\beta_{2 m}$ interdomain bond, thereby shifting the mechanical load originally borne by this bond to the $\alpha_{1} \alpha_{2}-\alpha_{3}$ joint to induce its partial unfolding, as observed in SMD simulations (5). As such, the MHC $\alpha_{3}$ domain would change its length and rotate about its C-terminus (Fig. $1 B$ ). We also assume that disruption of the $\alpha_{1} \alpha_{2}-\beta_{2 m}$ joint would result in tilting of the normal direction of TCR-pMHC bonding interface by an angle $\theta$ and shifting of mechanical load from the TCR V $\beta-C \beta$ joint to the $V \alpha-C \alpha$ joint, leading to partial unfolding of the $\mathrm{V} \alpha-\mathrm{C} \alpha$ joint (Fig. $1 B$ ). At the transition state, therefore, we treat the 
MHC $\alpha_{3}$ domain $\left(d_{\alpha 3}\right)$, the MHC $\alpha_{1} \alpha_{2}$ domains bound to the TCR V $\alpha \beta$ domains $\left(d_{\text {B.I }}\right)$, and the TCR C $\alpha \beta$ domains $\left(d_{\mathrm{C} \alpha \beta}\right)$ as three semi-rigid bodies connected by two unfolded peptide chains of the MHC $\alpha_{1} \alpha_{2}-\alpha_{3}$ joint $\left(d_{\mathrm{p}, \mathrm{MHC}}\right)$ and the TCR V $\beta-\mathrm{C} \beta$ joint $\left(d_{\mathrm{p}, \mathrm{TCR}}\right)($ Fig. $1 B$, right $)$. At the bound state, neither disruption of intramolecular bonds nor partial unfolding of interdomain joints was assumed to occur, as mentioned earlier, allowing the whole TCR-pMHC ectodomain complex to be modeled as one semi-rigid body $\left(d_{\mathrm{N}}\right)$.

Force-induced energy change: To derive an expression for the last term on the righthand side of Eq. 1, we model the semi-rigid bodies $d_{\mathrm{i}}\left(i=\mathrm{N}, \alpha_{3}\right.$, B.I., and C $\alpha \beta$ to denote the whole TCR-pMHC structure as well as its indicated domains) as three-dimensional freelyjointed chains (FJC) and employ polymer physics to obtain their force-extended length $d_{\mathrm{i}}(f)$ from their force-free length $d_{\mathrm{i}, \mathrm{c}}(22)$ (Supplemental Model Derivations, Eq. S1).

The assumed partial unfolding of the $\alpha_{1} \alpha_{2}-\alpha_{3}$ joint and the $\mathrm{V} \alpha-\mathrm{C} \alpha$ joint is based on suggestions from single-molecule optical tweezers (4) and magnetic tweezers (5) experiments as well as SMD simulations (5). We model these unstructured polypeptides as extensible worm-like chains (eWLC) and employ polymer physics to obtain their force-induced extension $d_{\mathrm{p}, \mathrm{i}}(f)(i=\mathrm{MHC}$ and TCR) from their force-free, folded state, which has zero length (23) (Supplemental Model Derivations, Eq. S3).

Upon projecting the various force-induced extensions described above onto the force axis, we obtain $z$ components of five contributions to the TCR-pMHC length increase at the transition state: extension of the $\mathrm{MHC} \alpha_{3}$ domain $\left(z_{\alpha 3}\right)$, unfolding of the $\mathrm{MHC} \alpha_{1} \alpha_{2}-\alpha_{3}$ interdomain joint $\left(z_{\mathrm{p}, \mathrm{MHC}}\right)$, extension of bonding interface that includes the MHC $\alpha_{1} \alpha_{2}$ domains bound to the TCR $\operatorname{V} \alpha \beta$ domains $\left(z_{\mathrm{B} . \mathrm{I}}\right)$, unfolding of the $\mathrm{V} \alpha-\mathrm{C} \alpha$ joint $\left(z_{\mathrm{p}, \mathrm{TCR}}\right)$, and extension of the TCR C $\alpha \beta$ domains $\left(z_{C \alpha \beta}\right)$ (Fig. $\left.1 B\right)$. Finally, we obtain: 


$$
\delta_{\mathrm{l}} \gamma(F)=\int_{0}^{F}\left[z_{\alpha 3}(f)+z_{\mathrm{p}, \mathrm{MHC}}(f)+z_{\mathrm{B} . \mathrm{I}}(f)+z_{\mathrm{p}, \mathrm{TCR}}(f)+z_{\mathrm{C} \alpha \beta}(f)-z_{\mathrm{N}}(f)\right] d f
$$

\section{Model characterization}

Model constants and parameters: The FJC model constants for the $1^{\text {st }}, 3^{\text {rd }}, 5^{\text {th }}$, and $6^{\text {th }}$ terms in the integrand on the right-hand side of Eq. 3 includes the force-free lengths $d_{\mathrm{i}, \mathrm{c}}$ and the elastic modulus of the folded globular domain $E_{\mathrm{c}}$, all of which is available from the literature. The $2^{\text {nd }}$ and $4^{\text {th }}$ terms are proportional the respective numbers of amino acids in the polypeptides of the partially unfolded MHC $\alpha_{1} \alpha_{2}-\alpha_{3}$ joint $\left(n_{\mathrm{p}, \mathrm{MHC}}\right)$ and TCR V $\alpha-\mathrm{C} \alpha$ joint $\left(n_{\mathrm{p}, \mathrm{TCR}}\right)$, which can be combined as the product of the total unfolded amino acid number $n^{*}=$ $n_{\mathrm{p}, \mathrm{MHC}}+n_{\mathrm{p}, \mathrm{TCR}}$, the average contour length per unfolded amino acid $l_{\mathrm{c}}$, and the extension per unit contour length $z_{\mathrm{u}, \mathrm{p}}(f)$. The eWLC model constants for $z_{\mathrm{u}, \mathrm{p}}(f)$ include the average persistence length per unfolded amino acid $l_{\mathrm{p}}$ and the elastic modulus of polypeptides $E_{\mathrm{p}}$ (Supplemental Table S1).

After applying model constraints and the approximating $\Delta G_{0}^{*} \sim \ln \left(k_{w} / k_{0}\right)$ where $k_{w}$ $\sim 10^{6} \mathrm{~s}^{-1}$ is known as the prefactor (Supplemental Model Derivations), the model parameters are reduced to 5: 3 structural parameters $\left(d_{\alpha 3}, \theta, n^{*}\right)$ and 2 biophysical parameters $\left(k_{0}, \delta_{0}^{*}\right)$, for describing dissociation of TCR-pMHC-I bonds. Unlike model constants obtained from the literature, we will determine these parameters by comparing the model predictions with experimental measurements, and by doing so, illustrate the ability of our model to use a relatively low number of parameters to capture the coarse-grained structure and conformational changes at the sub-molecular level during TCR-pMHC-I dissociation.

Model features and properties: To explore the general features and properties of the model, we plotted $\delta_{\mathrm{z}}$ vs $F$ for two $n^{*}$ values and a range of $\theta$ values as well as two $\theta$ values and a range of $n^{*}$ values (Fig. 1C). Conceptually, force-heightened energy barrier generates 
catch bonds and force-lowered energy barrier produces slip bonds (Fig. 1D, top left and right panels). Since $-\delta_{1} \gamma$ represents the energy input by force $F$ to the original energy landscape, a biphasic $\delta_{1} \gamma$ is required to create catch-slip bonds (Fig. 1D, top middle panel); correspondingly, $\delta_{\mathrm{z}}$ is required to have a root at positive $F$ where catch bond transitions to slip bond (Fig. $1 C$ ). The parameter regions capable of generating catch, catch-slip, and slip bonds are mapped on an $n^{*}-\theta$ phase diagram (Fig. 1D), showing that our model can describe catch-slop bond if and only if $n^{*}>0, \theta>0$, and $\delta_{1} \gamma(\infty)>0$ (Fig. $1 D$, top middle panel). In other words, catch-slip bonds require partial unfolding of the MHC $\alpha_{1} \alpha_{2}-\alpha_{3}$ and/or TCR V $\alpha-C \alpha$ joints and tilting of the TCR-pMHC bonding interface, a prediction consistent with previous results of SMD simulations and single-molecule experiments (5).

For single-bond dissociation from a single bound state along a single pathway, the reciprocal dissociation rate should equal to the average bond lifetime. Regardless of the bond type, the reciprocal zero-force off-rate controls the $y$-intercept of the bond lifetime vs force curves. We plotted the theoretical bond lifetime (normalized by its zero-force value) $k_{0} / k$ vs force $F$ for a range of $n^{*}, \theta$, and $\delta_{0}^{*}$ to examine how the model parameters control the bond lifetime vs force profile (Fig. $1 E$ ). Consistent with Fig. $1 C$ - $D$, only if $n^{*}>0$ and $\theta>0$ can our model describe catch-slip bond. Increasing the tilting angle $\theta$ results in more pronounced catch-slip bonds with longer lifetimes that peak at higher forces (Fig. 1E, upper panel). By comparison, increasing $\delta_{0}^{*}$ changes the level of slip-only bonds if $n^{*}=0$ and $\theta=0$, but prolongs bond lifetime of catch-slip bonds (until cross-over at a higher force) without changing the force where lifetime peaks if $n^{*}>0$ and $\theta>0$ (Fig. 1E, lower panel).

To understand physically how our model describes catch-slip bonds, we plotted the energy landscape using Eq. 1 (Fig. $1 F$ ). Setting $\theta=0$ in the upper panel generated a family of $\Delta G$ vs $\delta_{\mathrm{l}}$ curves where the energy barrier is suppressed monotonically with increasing force, 
indicating a slip-only bond (Fig. $1 F$, upper panel). By comparison, setting $\theta>0$ results in a family of $\Delta G$ vs $\delta_{\mathrm{l}}$ curves where the energy barrier height is initially raised, then lowered, by increasing force, indicating a catch-slip bond (Fig. $1 F$, lower panel). We also examine how the transition state location (Fig. 1G, upper row) and energy barrier height (Fig. 1G, lower row) change with force for a range of $\theta$ and $\delta_{0}^{*}$ values that give rise to slip-only bonds and catchslip bonds. Noticeably, at fixed $\theta$ value, both rates by which the transition state location and the energy barrier height change with force are accelerated by increasing $\delta_{0}^{*}$ (Fig. $1 G$, right column), suggesting that this parameter can be used as a measure for force sensitivity. Interestingly, increasing $\theta$ dampens the decrease in both the transition state location and energy barrier height with force at higher values, suggesting that the tilting angle controls the range at which force sensitivity can last (Fig. 1G, left column).

\section{Model validation}

Model's capability to fit data: To test our model's validity, we used it to reanalyze data of various TCRs forming catch-slip bonds and slip-only bonds with their respective panels of pMHCs. These include three datasets published by us: 1) murine OT1 TCR expressed on either primary naïve $\mathrm{CD}^{+} \mathrm{T}$ cells or $\mathrm{CD} 4^{+} \mathrm{CD} 8^{+}$thymocytes, which interacted with peptides presented by a MT MHC (H2-K $\left.\mathrm{K}^{\mathrm{b}} \alpha 3 \mathrm{~A} 2\right)$ to abolish CD8 co-engagement $(2,9)$ (Fig. $2 A$ and Fig. $\mathrm{S} 1 A)$; 2) WT or MT murine $2 \mathrm{C}$ TCRs either expressed on primary naïve $\mathrm{CD}^{+} \mathrm{T}$ cells or reexpressed on CD8- hybridoma cells, which interacted with peptides presented by $\mathrm{H} 2-\mathrm{K}^{\mathrm{b}} \alpha 3 \mathrm{~A} 2$ (for $\mathrm{CD}^{+}$primary $\mathrm{T}$ cells) or $\mathrm{H} 2-\mathrm{K}^{\mathrm{b}}$ (for $\mathrm{CD} 8^{-}$hybridoma cells) to ensure no $\mathrm{CD} 8$ contribution, or by $\mathrm{H} 2-\mathrm{L}^{\mathrm{d}}(\mathrm{m} 31)$, a different MHC allele from $\mathrm{H} 2-\mathrm{K}^{\mathrm{b}}$ (Fig. $2 B$, Fig. $\mathrm{S} 1 B$ and $C$ ) (5); and 3) WT and three MT human 1G4 TCRs were re-expressed on hybridoma cells, which interacted with the melanoma peptide NY-ESO-1 bound to HLA-A2 (Fig. S1D) (5). In addition, we fitted our model to two datasets published by other labs: 1) soluble mouse N15 TCR $\alpha \beta$ interacted 
with VSV and two MT peptides bound to $\mathrm{H}_{2}-\mathrm{K}^{\mathrm{b}}$ (Fig. S1E) (4) (Fig. S1E) and 2) four mouse TCRs expressed on hybridomas interacted with $\mathrm{NP}_{366}$ bound to the $\mathrm{D} 227 \mathrm{~K}$ mutant of $\mathrm{H}-2 \mathrm{D}^{\mathrm{b}}$ to prevent CD8 binding (11) (Fig. S1F). Gratifyingly, the theoretical reciprocal force-dependent off-rate $1 / k(F)$ fits all 33 experimental bond lifetime vs force curves well (Fig. $2 A$ and $B$, Fig. S1), demonstrating our model's capability to describe a wide range of data.

Characterization of force-lifetime relationship: Previous work reported qualitative correlations between the TCR bond type, i.e., catch-slip bond vs slip-only bond, with the biological activity of the peptide to induce T cell activation, i.e., pMHC potency $(2,5,7,9)$. To reduce data representation and extract more information quantitatively from the bond lifetime vs force data, we defined several metrics from their model fit for each TCR-pMHC system and examined their correlation with $\mathrm{T}$ cell activation induced by a given interaction, using the OT1 system as an example because the quantitative ligand potency data are available $(2,24)$. We measured the peak bond lifetime, $t_{\text {peak }}$, and the change, $\Delta t$, from $t_{\text {peak }}$ to the forcefree bond lifetime, $t_{0}=1 / k_{0}$ (Fig. $2 A$, left panel). We found the relative metric $\Delta t$ more suitable for comparison across different TCR systems, and better correlates with ligand potency, than the absolute counterpart $t_{\text {peak }}$ (Fig. $2 C$ ). Although the force where catch-slip bond lifetime peaks, $F_{\text {opt }}$, occurs in a narrow range $(10-20 \mathrm{pN})$, the force increment, $\Delta F$, from $F_{\text {opt }}$ to the level where bond lifetime returns to $t_{0}$ defines the force span of a catch-slip bond (Fig. $2 A$, left panel). One scaled parameter, $L=\Delta t / t_{\text {peak }}$ (relative length of lifetime), does (Fig. $2 D$, left panel), but the other, $B=\Delta F / F_{\text {opt }}$ (relative breadth of lifetime), does not (Fig. $\mathrm{S} 2 A$ ), correlate with ligand potency well. Remarkably, the combined scaled parameter termed the catch bond intensity, $I=L /(1+B)$, correlates best with the ligand potency across 
different TCR systems (Fig. 2D, right panel and 2E), supporting its usefulness and reasonableness as a metric of reduced data representation for catch and slip bonds.

Model parameters' correlation to ligand potency: It seems reasonable to test the validity of our model by examining the possible correlation of (or the lack thereof) the model parameters with features of the biological system, e.g., the ligand potency. The rationale is that if its parameters are capable of capturing and predicting such biological features, then the model would be more meaningful and useful than merely a curve fitting tool. Therefore, we plotted the tilted angle of the bonding interface $\theta$, the number of the unfolded amino acids $n^{*}$, and the width of the zero-force free-energy well $\delta_{0}^{*}$ that best-fit the force-lifetime curves of OT1, 2C, N15, TRBV TCRs interacting with their corresponding panels of pMHCs (Fig. 2F). Gratifyingly, we observed good correlations between all three model parameters and the peptide potency for all published data of TCR-pMHC-I catch-slip bonds and slip-only bonds from four independent laboratories $(2,4,5,9,11)$

In a previous study, we mutated residues in the $2 \mathrm{C}$ or $1 \mathrm{G} 4 \mathrm{TCR}$ and/or their corresponding pMHCs to alter bond profiles as predicted by SMD simulations, which was confirmed by experiment (5). We therefore fitted our model to the force-lifetime curves of these mutant TCR-pMHC bonds, which evaluated the best-fit model parameters, $\delta_{0}^{*}, \theta$, and $n^{*}$ (Figs. $3 A, \mathrm{~S} 2 B$, and $\mathrm{S} 2 C$ ). In the absence of other functional data, we took an indirect approach to examine their correlations with the catch bond intensity $I$ of these bond lifetime vs force curves (Fig. $3 B$ ) since both $I$ and model parameters correlate with the peptide potency (Fig. $2 E$ and $F$ ). Results are exemplified by the $\delta_{0}^{*}, \theta$, and $n^{*}$ vs $I$ plots, which are graphed together with the data without TCR and MHC mutations that already showed functional correlates. For the WT OT1, 2C, N15, and TRBV TCRs interacting with their corresponding panels of pMHCs, $\delta_{0}^{*}$ (Fig. $3 C$ ), and to a lesser extent, $\theta$ (Fig. S2D) and $n^{*}$ (Fig. S2E), correlate 
with $I$ that predicts peptide potency (blue-open symbols). Remarkably, for the $2 \mathrm{C}$ and $1 \mathrm{G} 4$ TCRs specifically mutated to alter bond profiles with the corresponding WT or mutant MHCs presenting the same agonist peptide, their best-fit $\delta_{0}^{*}$, and to a lesser extent, $\theta$ and $n^{*}$, also correlate well with the $I$ value (Figs. $3 C, \mathrm{~S} 2 D$ and $\mathrm{S} 2 E$, green-closed symbols). These results support our model's validity and suggest that $\theta, n^{*}$, and $\delta_{0}^{*}$ may serve as catch bond parameters to discriminate antigen quantitatively. Interestingly, $1 / k_{0}$ shows a negative correlation with $I$ (Fig. S2F), consistent with reports that zero-force bond lifetime does not correspond to ligand potency in these cases $(2,5,24)$.

Comparison between coarse-grained and all-atom models: Bonding interface tilting has been observed to be associated with changes in the number of hydrogen bonds bridging the TCR and pMHC molecules as they were forced to unbind in SMD simulations (5). Therefore, we investigated whether, and if so, how well the tilting angle would correlate with the change of hydrogen bonds between TCR and MHC. Remarkably, $\theta$ was found to be proportional to the net change in the total number of hydrogen bonds at the bonding interface (Figs. $3 D$ and S3). This finding is intuitive and supports the validity of our coarse-grained model because it is able to recapitulate the results of all-atom SMD simulations (5).

Classification of bond types by clustering analysis on phase diagrams: In Fig. $1 E-G$ we have explored the model parameter space to identify regions that correspond to slip-only bonds and catch-slip bonds. Here we examined whether, and if so, how parameters that best-fit different experimental bond types map onto different regions of the parameter space. Since the model has four parameters, $\theta, \delta_{0}^{*}, d_{\alpha 3}$, and $n^{*}\left(k_{0}\right.$ is not considered because if its lack of correlation with catch bond intensity), we analyzed their clustering and projected their values in the 4D parameter space onto three phase diagrams spanning the $\theta-\delta_{0}^{*}$ (Fig. $3 E$ ), $\theta-d_{\alpha 3}$ (Figs. $3 F$ and S4), and $\delta_{0}^{*}-n^{*}$ (Fig. $\left.3 G\right) 2 \mathrm{D}$ space. Clustering analysis of the model parameters that 
best-fit 36 published TCR-pMHC bond lifetime vs force curves (Fig. S5) shows three distinct clusters in the $\delta_{0}^{*}$ vs $\theta$ and $\theta$ vs $d_{\alpha 3}$ plots as well as $\delta_{0}^{*}$ and $\theta$ vs $n^{*}$ plots (Fig. 3E-G), which classify the TCR-pMHC interactions into slip-only (SO), weak catch-slip (WC) and strong catch-slip (SC) bonds, which correspond to weak, intermediate, and strong potencies for pathogenic peptides and their variants. Whereas transition in bond type from SO to WC and SC requires monotonical increase in $\theta$ and $n^{*}$ (Fig. $3 F$ and $G$ ), the corresponding change in $\delta_{0}^{*}$ is non-monotonic (Fig. $3 E$ and $G$ ). SO bonds show small $n^{*}, \delta_{0}^{*}$, and $\theta$ values. WC and SC bonds observed from published experiments are best-fitted by similar $n^{*}$ ( 5 for WC and 7 for $\mathrm{SC)}$ but oppositely ranked $\delta_{0}^{*}$ and $\theta$ values. To change from WC to SC bonds requires decreasing $\delta_{0}^{*}$ (from 2.7 to $1.9 \mathrm{~nm}$ ) but increasing $\theta$ (from 14 to $32^{\circ}$ ) (Fig. $3 G$ ). We also performed principal component analysis and calculated the Mahalanobis distances of the principal axes for the three bond types (25), which are statistically separated in the catch bond intensity vs Mahalanobis distance plot (Fig. 3H). Interestingly, WC and SC bonds show distinct conformational changes despite their similar $I$ values measured from the force-lifetime curves. The corresponding structural features of these three types of bonds are depicted in Fig 3I, which have been observed in our previous SMD studies (5).

\section{Model for TCR catch bonds with class II pMHC}

MHC class II differs from class I in three main aspects (comparing Fig. $1 A$ and Fig. 4A): (1) MHC-I has three $\alpha$ domains and a $\beta_{2 m}$ domain whereas MHC-II has two $\alpha$ and two $\beta$ domains. (2) MHC-I anchors to the T cell membrane through a single linker to the $\alpha_{3}$ domain. The $\beta_{2 \mathrm{~m}}$ domain attaches to the $\alpha_{3}$ domain instead of anchoring to the T cell membrane directly. By comparison, MHC-II anchors to the membrane through two linkers, one to the $\alpha_{2}$ domain and the other to the $\beta_{2}$ domain (3). The peptide is presented by the $\alpha_{1}-\alpha_{2}$ domains of MHC-I but the $\alpha_{1}-\beta_{1}$ domains of MHC-II. These structural differences alter how forces are supported 
by and transmitted through, and induce conformational changes in, the TCR complexes with pMHC-I vs pMHC-II. Thus, it was necessary to modify the model in order for it to describe TCR catch and slip bonds with MHC-II, which was done by using a different $\delta_{1} \gamma(F)$ expression than Eq. 3 (Supplemental Model Derivations, Section B). This modification assumes force-induced partial unfolding and stretching of the TCR V $\alpha-C \alpha$ joint and the MHC $\alpha_{1}-\alpha_{2}$ and $\beta_{1}-\beta_{2}$ joints during dissociation, which results in tilting of the bonding interface (Fig. $4 A$ and $B)$.

In the class II model, the same parameters $\delta_{0}^{*}, n^{*}$, and $\theta$ are used but the MHC contribution to $n^{*}$, i.e., $n_{\mathrm{p}, \mathrm{MHC}}$, represents the average number of amino acids in the polypeptides of the partially unfolded MHC-II $\alpha_{1}-\alpha_{2}$ and $\beta_{1}-\beta_{2}$ joints instead of the MHC-I $\alpha_{1} \alpha_{2}-\alpha_{3}$ joint, and the relationships between $\theta$ to other structural parameters are also different from the class I model (Fig. $4 B$ and Supplemental Model Derivation, Section B). Like the class I model, the $k_{0} / k$ vs $F$ plots for a range of $n^{*}, \theta$, and $\delta_{0}^{*}$ in Fig. $4 C$ show similar features to Fig. $1 F$ and meet our objective of being capable of describing catch-slip bonds if and only if $n^{*}>0$ and $\theta \geq 0$. Unlike the class I model, a much smaller $\theta$ value $\left(<10^{\circ}\right)$ is seen in the class II model (compared Fig. $4 C$ and Fig. S6 with Fig. $1 E$ and $G$ ), indicating the main conformational change responsible for TCR-pMHC-II catch-slip bond is unfolding rather than tilting. The validity of this model is supported by its excellent fitting to our published data of mouse WT or MT TCRs on naïve T cells (3.L2) or hybridomas (2B4) interacting with their respective p:I- $E^{\mathrm{k}}$ ligands $(3,26)$ (Fig. 4D, Tables S4 and S5). The four metrics of the TCRpMHC-II bond lifetime vs force curves $\Delta t, L, I$, and $t_{\text {peak }}$ correlate well with the published peptide potency $(27,28)$ (Figs. $4 E$ and S7). Since mutations on the $2 \mathrm{~B} 4$ TCR were designed to perturb its $\mathrm{C} \alpha \beta$ interaction with the CD3 subunits, their effect on the bond profile and $\mathrm{T}$ cell 
function might be weaker than the effects of the altered peptide ligands for the 3.L2 TCR (3, 26). Nevertheless, the correlations between ligand potency and the three model parameters $\theta$, $n^{*}$, and $\delta_{0}^{*}$ for the $2 \mathrm{~B} 4$ system are comparable to those for the 3.L2 system (Fig. $4 F$ ), supporting the ability of the metrics of the bond profile and the model parameters to recognize the change in the TCR-CD3 ectodomain interaction in addition to the ability to discriminate antigen. These properties are desirable, intuitive, and are consistent with the parallel properties found in the class I model. Similar to the class I model parameters, $\delta_{0}^{*}$ correlates well with the catch bond intensity for the pooled results from all published class II data (Fig. $4 G$, left), but $\theta$ and $n^{*}$ correlate less well with $I$ (Fig. 4G, middle and right). Thus, the validity of the class II model is further supported by the faithful mapping of the relationship between biophysical measurements of catch and slip bonds and biological activities of the TCR-pMHC-II interactions onto a relationship between model parameters and biological function.

\section{Cross-examination of class I model against class II data and vice versa}

Upon examining the catch-slip and slip-only bond lifetime vs force curves in Figs. $2 A$, $2 B, 4 D$ and $\mathrm{S} 1$, it became apparent that the data seem very similar regardless of whether they are for class I or class II pMHC. Indeed, applying the class I model to the class II data and vice versa reveals that both models are capable of fitting both data sets well (Fig. S8) and produce statistically indistinguishable goodness-of-fit measures (Fig. S9). This is not surprising because both models have five fitting parameters and the bond lifetime vs force curves have relatively simple shapes. Nevertheless, fitting the same data by different models returns different parameter values depending on the model used, because the two models are constructed based on different structures and force-induced conformational changes of the TCR-pMHC complexes. Therefore, we asked whether the best-fit model parameters were capable of distinguishing data from the two classes of pMHCs and of telling whether a correct model was 
used to analyze the data of the matched MHC class. To answer these questions, we plotted $\delta_{0}^{*}$ vs $I$ (Fig. $5 A$ and $B$ ) and $\delta_{0}^{*}$ vs $n^{*}$ (Fig. $5 D$ and $E$ ) using values of the two models that best-fit the data of OT1, 2C, 1G4, N15, and TRBV TCRs interacting with their respective panels pMHC-I ligands (Fig. 5A and $D$ ) as well as 3.L2, 2B4, and E8 TCRs interacting with their respective panels of pMHC-II ligands (Fig. $5 B$ and $E$ ). Surprisingly, the dependency of $\delta_{0}^{*}$ on $I$ is 2 3-fold stronger (i.e., steeper slope) (Fig. 5C), indicating a greater discriminative power of ligand potency for the matched than the mismatched cases. Furthermore, it is well-known that the average contour length per a single amino acid $l_{\mathrm{c}}$ is $\sim 0.4 \mathrm{~nm}(17,29,30)$, which sets the biophysical limit for the slope of $\delta_{0}^{*}$ vs $n^{*}$ plots. Indeed, we found that the slopes of the $\delta_{0}^{*}$ vs $n^{*}$ plots are within this limit for both model fitting the class I data (Fig. $5 F$ ). For the class II data, the slope of the $\delta_{0}^{*}$ vs $n^{*}$ plot reaches this limit for the matched case but exceeds this limit for the mismatched case (Fig. $5 F$ ). Moreover, the goodness-of-fit $\left(R^{2}\right)$ values of the linear fit to the $\delta_{0}^{*}$ vs $I$ (Fig. $5 C$ ) and $\delta_{0}^{*}$ vs $n^{*}$ (Fig. $5 F$ ) data are much greater for the matched than the mismatched cases, indicating more appropriate models for the data in the matched than the mismatched cases. Indeed, the $R^{2}$ value for fitting the class I data by the class II model is too small to be statistically reasonable, therefore telling the mismatch between the model and the data. These results indicate that the model parameters are capable of distinguishing data from the two classes of pMHCs.

\section{Discussion}

In this work we developed two mathematical models for TCR catch bonds, one with class I and the other with class II pMHC, based on Kramer's kinetic theory and accounted for the $3 \mathrm{D}$ coarse-grained structures, molecular elasticity, and conformational changes of the TCR-pMHC-I/II complexes. Force-induced conformational changes of TCR-pMHC-I 
complexes have been observed or suggested by single-molecule experiments and SMD simulations $(4,5)$. Parameterizing these conformational changes by the number of unfolded amino acids $n^{*}$ and the bonding interface titling angle $\theta$ in the class I model allows us to explain mechanistically and quantitatively the TCR-pMHC-I catch-slip and slip-only bonds. Indeed, the criteria for catch-slip bond are $n^{*}>0$ and $\theta>0$; the greater their values the more pronounced the catch bond. Importantly, the validity of the class I model has been supported by its capability to fit all force-lifetime data published to date and by the correlation between the best-fit model parameters and the available biological activitiy data induced by the TCRpMHC-I interactions.

By comparison, the respective ranges of $n^{*}$ and $\theta$ for the class II model are smaller, consistent with the sturdier structure of pMHC-II (19). Neither experimental nor simulation data have been reported to suggest force-induced conformational changes in the TCR-pMHCII structure. Our class II model has also been tested by all published data and their best-fit parameters also correlate well with the biological activities induced by the TCR-pMHC-II interactions. Furthermore, the validity of models of both classes has been supported by the findings that the best-fit model parameter $\delta_{0}^{*}$ correlates with the catch bond intensity $I$ and that the $\delta_{0}^{*}$ vs $I$ and $\delta_{0}^{*}$ vs $n^{*}$ plots have more appropriate slopes and $R^{2}$ values when the model matches than mismatches the data.

A strength of our agent-based models lies in their ability to incorporate many different ideas and knowledge into a simple 1D formulation. This simplicity facilitates model application to both class I and II experimental systems, enables quantitative interpretation of TCR-pMHC bond lifetime vs force profiles, expresses biological functions by biophysical measurements, and suggests structural mechanisms of how the TCR mechanotransduction machinery might work. However, the 1D simplification is also a weakness because theoretically these models 
can and only can describe single-step dissociation from a single-state along a singledissociation path, implicitly assuming that there is only a single energy barrier. Although some catch-slip and slip-only bonds can be described by such simple models (e.g., (3)), more complicated TCR-pMHC bonds has been reported. These are evidenced by the multiexponential bond lifetime distributions at constant forces, which have been fitted by datadriven multi-state and/or multi-pathway models $(2,5)$. To address this weakness, future studies may extend the present $1 \mathrm{D}$ model to $2 \mathrm{D}$ to enable proper description of multi-exponential bond survival probabilities.

An objective of the present work is to explore the extent to which 1D models can describe experimental data with a minimal set of meaningful parameters. Our parameters consider coarse-grained structural features and relate catch and slip bonds to specific forceinduced conformational changes of the TCR-pMHC complex. This approach should be extendable to the modeling of other receptor-ligand systems of different structural features but also form catch and slip bonds, such as selectins $(13,31,32)$, integrins (33-38), cadherin (39), Fc $\gamma$ receptor (40), notch receptor (41), platelet glycoprotein $\operatorname{Ib} \alpha(42,43)$, FimH (44), actin with myosin (45), actin with actin $(46,47)$, cadherin-catenin complex with actin (48), vinculin with F-actin (49), and microtubule with kinetochore particle (50).

We introduced the catch-bond intensity $I$ as a dimensionless scaled metric for the bond lifetime vs force curve and generated four model parameters that describe the curve's geometric features. Upon analyzing all catch-slip and slip-only bond profiles (a total of 48 curves) published to date by four independent laboratories $(2-5,9,11,26,51,52)$, we found that these quantities do a better job to predict TCR function than any other quantities. This finding explains how force amplifies TCR signaling and antigen discrimination, because $I$ is defined 
by a force curve and $n^{*}$ and $\theta$ only predict signaling when they assume none-zero values at $F$ $>0$.

A recent study showed surprising features of reversed-polarity TRBV TCRs such that binding of $\mathrm{NP}_{366}: \mathrm{H}-2 \mathrm{D}^{\mathrm{bD} 227 \mathrm{~K}}$ to TCRs B13.C1 and B17.C1 induces T cell signaling, whereas binding of the same pMHC to B17.R1 and B17.R2 TCRs does not (11). Despite that the former two TCRs form catch-slip bonds with $\mathrm{NP}_{366}: \mathrm{H}-2 \mathrm{D}^{\text {bD227K }}$ and the latter two TCRs form sliponly bonds, the authors suggested that the signaling capability of the B13.C1 and B17.C1 TCRs could not be attributed to their force-prolonged bond lifetimes because the B17.C1 TCR-H$2 \mathrm{D}^{\mathrm{bD} 227 \mathrm{~K}}$ bond was shorter-lived than the B17.R2 TCR-NP $366: \mathrm{H}-2 \mathrm{D}^{\mathrm{bD} 227 \mathrm{~K}}$ bond across the entire force range tested. Even at $9.4 \mathrm{pN}$, which is $F_{\text {opt }}$ for the former with a $t_{\text {peak }}=0.61 \mathrm{~s}$, the latter lived $2.48 \mathrm{~s}$ on average, and the longest lifetime of the latter is $t_{0}=2.83 \mathrm{~s}$ occurred at zero force (11). The authors hypothesize that the TCR-pMHC docking orientation, which is "canonical" for the B13.C1 and B17.C1 TCRs but "reversed" for the B17.R1 and B17.R2 TCRs, underlies the signaling outcomes by directing the position of Lck relative to the CD3. Even without knowing the docking orientation, however, our model parameters are capable of determining the signaling outcomes. Indeed, our analysis correctly maps the data of the B13.C1 and B17.C1 TCRs onto the high peptide potency region and the data of the B17.R1 and B17.R2 TCRs onto the low peptide potency region of the $\delta_{0}^{*}$ vs $I$ (Fig. $3 C$ ), $\delta_{0}^{*}$ vs $\theta$ (Fig. $3 E$ ), and $\theta$ vs $d_{\alpha 3}$ (Fig. $3 F$ ) phase diagrams. Thus, by mechanistically modeling the effect of force on bond dissociation, TCR signaling and antigen discrimination can be predicted by the model parameters.

The success in our model applications indicate that the conformational changes assumed in the models may be important to the TCR triggering, therefore suggesting testable hypotheses for future studies designed to investigate the inner workings of the TCR 
mechanotransduction machinery, e.g., to extend and/or revise models regarding how TCR signaling is triggered. One of the TCR triggering models involves conformational changes and catch bond formation (53-55). Our structure-based models relate catch and slip bonds to TCRpMHC conformational changes. For the class I model, the parameterized structural changes include force-induced disruption of the MHC $\alpha_{1} \alpha_{2}-\beta_{2 \mathrm{~m}}$ interdomain bond, partial unfolding of the $\alpha_{1} \alpha_{2}-\alpha_{3}$ joint, tilting of the normal direction of TCR-pMHC bonding interface, and partial unfolding of the $\mathrm{V} \alpha-\mathrm{C} \alpha$ joint. For the class II model, these are primarily limited to the forceinduced partial unfolding of the MHC-II $\alpha_{1}-\alpha_{2}$ and $\beta_{1}-\beta_{2}$ joints as well as the V $\alpha-\mathrm{C} \alpha$ and V $\beta$ $\mathrm{C} \beta$ joints. Besides these, one additional conformational change observed in the SMD simulations of TCR $\alpha \beta-$ pMHC dissociation is unfolding of the connecting peptides between the TCR $\alpha \beta$ ectodomain and transmembrane domain (5). We chose not to include this conformational change in our models because such unfolding would likely be prevented by the interaction of the $\mathrm{C} \alpha \beta$ with the CD3 subunits (26). Consistent with this assumption, the experimental data used for model fitting to evaluate conformational change parameters $\left(n^{*}\right.$ and $\theta$ ) are those of pMHC bonds with TCR $\alpha \beta$ ligand binding subunits that are complexed with the CD3 signaling subunits (except for the N15 TCR $\alpha \beta$ case). Indeed, our previous work found that catch bonds of purified TCR $\alpha \beta$ were altered from those of cell surface TCR interacting with the same pMHCs $(51,52)$, which is reflected by their changed model parameters (Fig. $\mathrm{S} 10)$. As such, the TCR $\alpha \beta$ conformational changes predicted by our models provide boundary conditions for possible CD3 conformational changes in the TCR-CD3 complex to be considered in future TCR triggering models. Another boundary conditions to be considered by future studies should be those imposed by the coreceptor CD4 and CD8, as the co-ligation of 
the coreceptor prolongs bond lifetimes, amplifies catch bonds, and may even changes slip-only bonds to catch-slip bonds $(7,9,52)$.

Our models allow us to develop working hypotheses regarding how $\mathrm{T}$ cell function is regulated through structural modulations of catch and slip bonds. For example, our class I model predicts that strengthening of the $\alpha_{1} \alpha_{2}-\beta_{2}$ interdomain bond would weaken the TCRpMHC catch bond, which would in turn reduce $\mathrm{T}$ cell activation. This prediction has been supported by our published data that somatic mutations in HLA-A2 found in some cancer patients impair TCR-pHLA-A2 catch bonds, which may explain the suppressed anti-tumor T cell immunity (5). More interestingly, our models pave the way for engineering of TCR function for tumor immunotherapy by modulating the TCR catch and slip bonds through alteration of its structures. For example, mutations that weaken the $\mathrm{V} \alpha-\mathrm{C} \alpha$ joint are predicted to strengthen TCR catch bonds and enhance T cell effector function, which suggests a strategy that may be more advantageous compared to mutations at the pMHC docking interface because mutations at the $\mathrm{V} \alpha-\mathrm{C} \alpha$ joint would alter function for any TCR specificity so it may be effective to many tumor antigens. By comparison, mutations at the TCR binding interface may be applicable to only a specific pMHC, but may also be riskier in terms of cross-reactivity to self-pMHCs. Thus, rational design guided by catch bond models may provide new TCR engineering strategies that warrant future studies.

\section{Materials and Methods}

The model developments, characterization, and validation are described in the main text with more details in the Supplemental Model Derivations. Model fitting to experimental data was done by nonlinear curve-fitting in the least-squares sense using the LevenbergMarquardt algorithm. Statistical and clustering analyses were done using Bayesian statistics and Lloyd's algorithm, respectively. All analyses were done using MATLAB. 


\section{References}

1. A. H. Courtney, W. L. Lo, A. Weiss, TCR Signaling: Mechanisms of Initiation and Propagation. Trends Biochem Sci 43, 108-123 (2018).

2. B. Liu, W. Chen, B. D. Evavold, C. Zhu, Accumulation of dynamic catch bonds between TCR and agonist peptide-MHC triggers T cell signaling. Cell 157, 357-368 (2014).

3. J. Hong et al., Force-regulated In situ TCR-peptide-bound MHC class II kinetics determine functions of CD4+ T cells. Journal of immunology 195, 3557-3564 (2015).

4. D. K. Das et al., Force-dependent transition in the T-cell receptor beta-subunit allosterically regulates peptide discrimination and $\mathrm{pMHC}$ bond lifetime. Proceedings of the National Academy of Sciences of the United States of America 112, 1517-1522 (2015).

5. P. Wu et al., Mechano-regulation of Peptide-MHC Class I Conformations Determines TCR Antigen Recognition. Mol Cell 73, 1015-1027 e1017 (2019).

6. Y. Feng et al., Mechanosensing drives acuity of alphabeta T-cell recognition. Proceedings of the National Academy of Sciences of the United States of America 114, E8204-E8213 (2017).

7. L. V. Sibener et al., Isolation of a Structural Mechanism for Uncoupling T Cell Receptor Signaling from Peptide-MHC Binding. Cell 174, 672-687 e627 (2018).

8. T. W. McKeithan, Kinetic proofreading in T-cell receptor signal transduction. Proceedings of the National Academy of Sciences of the United States of America 92, 5042-5046 (1995).

9. J. Hong et al., A TCR mechanotransduction signaling loop induces negative selection in the thymus. Nat Immunol 19, 1379-1390 (2018).

10. Y. Liu et al., DNA-based nanoparticle tension sensors reveal that T-cell receptors transmit defined $\mathrm{pN}$ forces to their antigens for enhanced fidelity. Proceedings of the National Academy of Sciences of the United States of America 113, 5610-5615 (2016).

11. P. Zareie et al., Canonical T cell receptor docking on peptide-MHC is essential for T cell signaling. Science 372, (2021).

12. M. Dembo, D. C. Torney, K. Saxman, D. Hammer, The reaction-limited kinetics of membrane-to-surface adhesion and detachment. Proc. R. Soc. Lond. B Biol. Sci. 234, 55-83 (1988).

13. B. T. Marshall et al., Direct observation of catch bonds involving cell-adhesion molecules. Nature 423, 190-193 (2003).

14. M. Grandbois, M. Beyer, M. Rief, H. Clausen-Schaumann, H. E. Gaub, How strong is a covalent bond? Science 283, 1727-1730 (1999).

15. G. Bell, Models for the specific adhesion of cells to cells. Science 200, 618-627 (1978).

16. B. Liu, W. Chen, C. Zhu, Molecular force spectroscopy on cells. Annu. Rev. Phys. Chem. 66, 427-451 (2014).

17. S. Guo, A. K. Efremov, J. Yan, Understanding the catch-bond kinetics of biomolecules on a one-dimensional energy landscape. Communications Chemistry 2 , 30 (2019).

18. Jarrett J. Adams et al., T cell receptor signaling is limited by docking geometry to peptide-major histocompatibility complex. Immunity 35, 681-693 (2011). 
19. J. Rossjohn et al., T cell antigen receptor recognition of antigen-presenting molecules. Annual review of immunology 33, 169-200 (2015).

20. H. A. Kramers, Brownian motion in a field of force and the diffusion model of chemical reactions. Physica 7, 284-304 (1940).

21. O. K. Dudko, G. Hummer, A. Szabo, Intrinsic rates and activation free energies from single-molecule pulling experiments. Physical review letters 96, 108101 (2006).

22. S. B. Smith, L. Finzi, C. Bustamante, Direct mechanical measurements of the elasticity of single DNA molecules by using magnetic beads. Science 258, 1122-1126 (1992).

23. C. Bouchiat et al., Estimating the persistence length of a worm-like chain molecule from force-extension measurements. Biophysical journal 76, 409-413 (1999).

24. J. Huang et al., The kinetics of two-dimensional TCR and pMHC interactions determine T-cell responsiveness. Nature 464, 932-936 (2010).

25. S. Xiang, F. Nie, C. Zhang, Learning a Mahalanobis distance metric for data clustering and classification. Pattern Recognition 41, 3600-3612 (2008).

26. Z. Yuan et al., Cooperative ectodomain interaction among TCR $\alpha \beta$, CD $3 \gamma \varepsilon$, and CD3 $\delta \varepsilon$ enhances TCR mechanotransduction. (2021).

27. G. J. Kersh, P. M. Allen, Structural basis for T cell recognition of altered peptide ligands: a single $\mathrm{T}$ cell receptor can productively recognize a large continuum of related ligands. $J$ Exp Med 184, 1259-1268 (1996).

28. A. Natarajan et al., Structural Model of the Extracellular Assembly of the TCR-CD3 Complex. Cell Rep 14, 2833-2845 (2016).

29. J. C. Gebhardt, T. Bornschlogl, M. Rief, Full distance-resolved folding energy landscape of one single protein molecule. Proceedings of the National Academy of Sciences of the United States of America 107, 2013-2018 (2010).

30. L. J. Lapidus, P. J. Steinbach, W. A. Eaton, A. Szabo, J. Hofrichter, Effects of Chain Stiffness on the Dynamics of Loop Formation in Polypeptides. Appendix: Testing a 1-Dimensional Diffusion Model for Peptide Dynamics. J. Phys. Chem. B 106, 1162811640 (2002).

31. K. K. Sarangapani et al., Low force decelerates L-selectin dissociation from Pselectin glycoprotein ligand-1 and endoglycan. J. Biol. Chem. 279, 2291-2298 (2004).

32. A. M. Wayman, W. Chen, R. P. McEver, C. Zhu, Triphasic force dependence of Eselectin/ligand dissociation governs cell rolling under flow. Biophys. J. 99, 1166-1174 (2010).

33. W. Chen, J. Lou, C. Zhu, Forcing switch from short- to intermediate- and long-lived states of the alphaA domain generates LFA-1/ICAM-1 catch bonds. J. Biol. Chem. 285, 35967-35978 (2010).

34. Y. Chen et al., An integrin alphaIIbbeta3 intermediate affinity state mediates biomechanical platelet aggregation. Nat Mater 18, 760-769 (2019).

35. Y. Chen, H. Lee, H. Tong, M. Schwartz, C. Zhu, Force regulated conformational change of integrin alphaVbeta3. Matrix Biol 60-61, 70-85 (2017).

36. Y. I. Choi et al., Dynamic control of beta1 integrin adhesion by the plexinD1-sema3E axis. Proc. Natl. Acad. Sci. U.S.A. 111, 379-384 (2014).

37. F. Kong, A. J. Garcia, A. P. Mould, M. J. Humphries, C. Zhu, Demonstration of catch bonds between an integrin and its ligand. J Cell Biol 185, 1275-1284 (2009).

38. F. Rosetti et al., A Lupus-Associated Mac-1 Variant Has Defects in Integrin Allostery and Interaction with Ligands under Force. Cell Rep 10, 1655-1664 (2015). 
39. K. Manibog, H. Li, S. Rakshit, S. Sivasankar, Resolving the molecular mechanism of cadherin catch bond formation. Nature communications 5, 3941 (2014).

40. H. Nishi et al., Neutrophil FcgammaRIIA promotes IgG-mediated glomerular neutrophil capture via Ab1/Src kinases. The Journal of clinical investigation 127, 3810-3826 (2017).

41. V. C. Luca et al., Notch-Jagged complex structure implicates mechanical force in ligand discrimination. Science 355, 1320-1324 (2017).

42. L. Ju, J. F. Dong, M. A. Cruz, C. Zhu, The N-terminal flanking region of the A1 domain regulates the force-dependent binding of von Willebrand factor to platelet glycoprotein Ibalpha. The Journal of biological chemistry 288, 32289-32301 (2013).

43. T. Yago et al., Platelet glycoprotein Ibalpha forms catch bonds with human WT vWF but not with type 2B von Willebrand disease vWF. J. Clin. Invest. 118, 3195-3207 (2008).

44. O. Yakovenko et al., FimH forms catch bonds that are enhanced by mechanical force due to allosteric regulation. The Journal of biological chemistry 283, 11596-11605 (2008).

45. B. Guo, W. H. Guilford, Mechanics of actomyosin bonds in different nucleotide states are tuned to muscle contraction. Proceedings of the National Academy of Sciences of the United States of America 103, 9844-9849 (2006).

46. C. Y. Lee et al., Regulation of actin catch-slip bonds with a RhoA-formin module. Sci Rep 6, 35058 (2016).

47. C. Y. Lee et al., Actin depolymerization under force is governed by lysine 113:glutamic acid 195-mediated catch-slip bonds. Proc. Natl. Acad. Sci. U.S.A. 110, 5022-5027 (2013).

48. C. D. Buckley et al., Cell adhesion. The minimal cadherin-catenin complex binds to actin filaments under force. Science 346, 1254211 (2014).

49. D. L. Huang, N. A. Bax, C. D. Buckley, W. I. Weis, A. R. Dunn, Vinculin forms a directionally asymmetric catch bond with F-actin. Science 357, 703-706 (2017).

50. B. Akiyoshi et al., Tension directly stabilizes reconstituted kinetochore-microtubule attachments. Nature 468, 576-579 (2010).

51. B. Liu et al., The cellular environment regulates in situ kinetics of T-cell receptor interaction with peptide major histocompatibility complex. European journal of immunology 45, 2099-2110 (2015).

52. M. N. Rushdi et al., Cooperative binding of TCR and CD4 to pMHC enhances TCR sensitivity. bioRxiv, https://www.biorxiv.org/content/10.1101/2021.1111.1122.469547v469541 (2021).

53. Y. Feng, E. L. Reinherz, M. J. Lang, alphabeta T Cell Receptor Mechanosensing Forces out Serial Engagement. Trends Immunol 39, 596-609 (2018).

54. C. Zhu, W. Chen, J. Lou, W. Rittase, K. Li, Mechanosensing through immunoreceptors. Nature Immunology 20, 1269-1278 (2019).

55. R. A. Mariuzza, P. Agnihotri, J. Orban, The structural basis of T-cell receptor (TCR) activation: An enduring enigma. The Journal of biological chemistry 295, 914-925 (2020).

56. S. Guo et al., Structural-elastic determination of the force-dependent transition rate of biomolecules. Chem Sci 9, 5871-5882 (2018).

57. H. K. Choi et al., Watching helical membrane proteins fold reveals a common N-toC-terminal folding pathway. Science 366, 1150-1156 (2019). 
58. Y. Seol, J. Li, P. C. Nelson, T. T. Perkins, M. D. Betterton, Elasticity of short DNA molecules: theory and experiment for contour lengths of 0.6-7 microm. Biophysical journal 93, 4360-4373 (2007).

59. H. S. Chung, J. M. Louis, W. A. Eaton, Experimental determination of upper bound for transition path times in protein folding from single-molecule photon-by-photon trajectories. Proceedings of the National Academy of Sciences of the United States of America 106, 11837-11844 (2009).

60. J. Kubelka, J. Hofrichter, W. A. Eaton, The protein folding 'speed limit'. Curr Opin Struct Biol 14, 76-88 (2004).

61. W. Y. Yang, M. Gruebele, Folding at the speed limit. Nature 423, 193-197 (2003).

62. B. Schuler, E. A. Lipman, W. A. Eaton, Probing the free-energy surface for protein folding with single-molecule fluorescence spectroscopy. Nature 419, 743-747 (2002). 


\section{Acknowledgments}

We thank Baoyu Liu, Jinsung Hong, Peng Wu, and Tongtong Zhang for sharing their published BFP experimental data for re-analysis and model fitting.

\section{Funding:}

National Research Foundation grants of South Korea 2021R1A6A3A03038382 (H.K.C.)

National Natural Science Foundation of China grant 31971237 (W.C.)

National Natural Science Foundation of China grant 32090044 (J.L.)

National Institutes of Health grant U01CA214354 (C.Z.)

National Institutes of Health grant U01CA250040 (C.Z.)

National Institutes of Health grant R01CA243486 (C.Z.)

National Institutes of Health grant R01GM061126 (C.Z.)

\section{Author contributions:}

Conceptualization: H.-K.C., C.Z.

Methodology: H.-K.C., K.L., M.N.R., C.G., C.Z.

Investigation: H.-K.C., C.Z.

Visualization: H.-K.C.

Funding acquisition: H.-K.C., W.C., J.L., C.Z.

Writing-original draft: H.-K.C., K.L., C.Z.

Writing—review \& editing: H.-K.C., K.L., M.N.R., C.G., W.C., J.L., C.Z.

\section{Competing interests:}

Authors declare that they have no competing interests

\section{Data and materials availability:}

All data are included in the article and Supplemental Materials. Previously published bond lifetime data were re-analyzed for models fitting $(2-5,9,11,26,52)$. 
bioRxiv preprint doi: https://doi.org/10.1101/2022.01.17.476694: this version posted January 18,2022 . The copyright holder for this

\section{Figures}

A

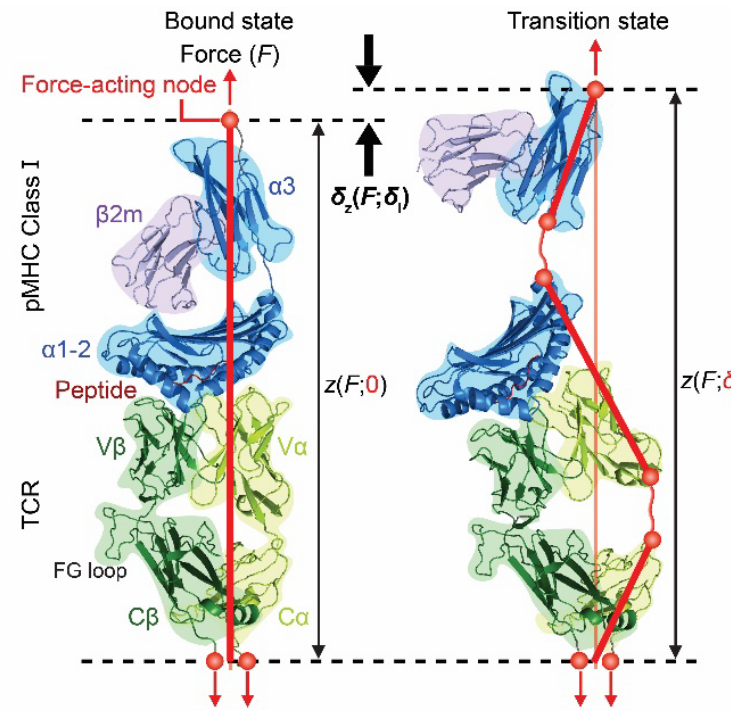

C
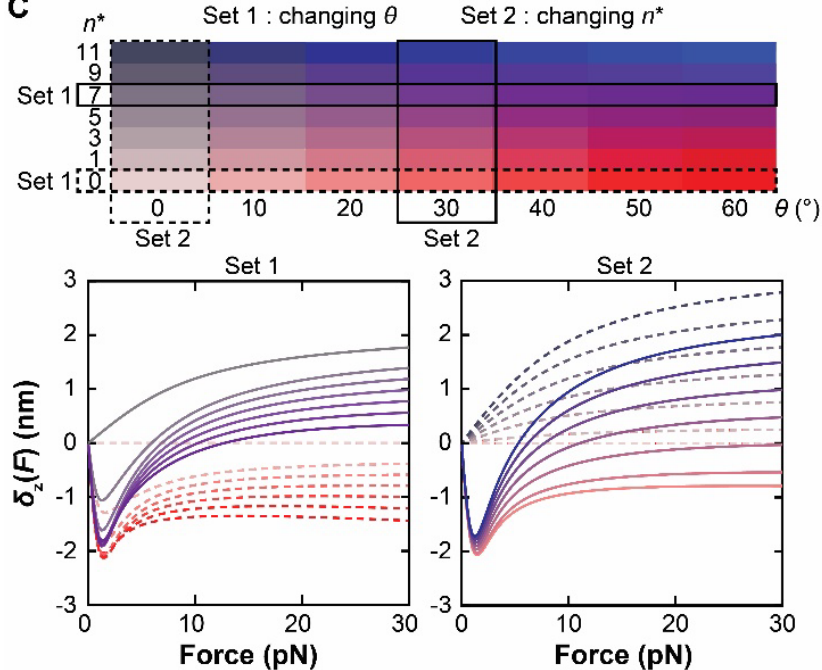

E

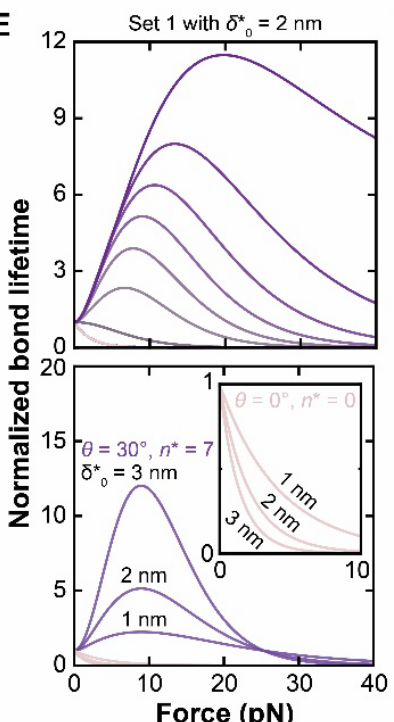

F 1-D free-energy landscape
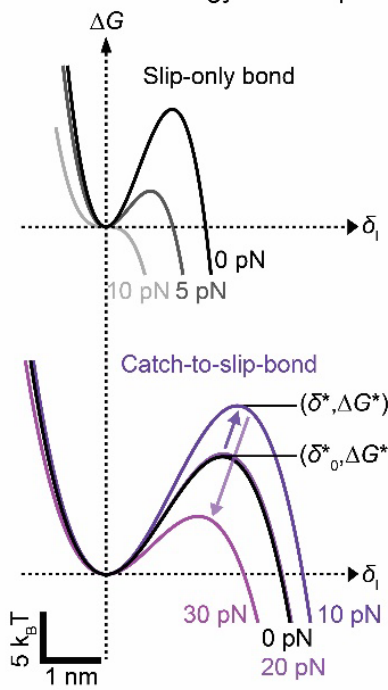

B

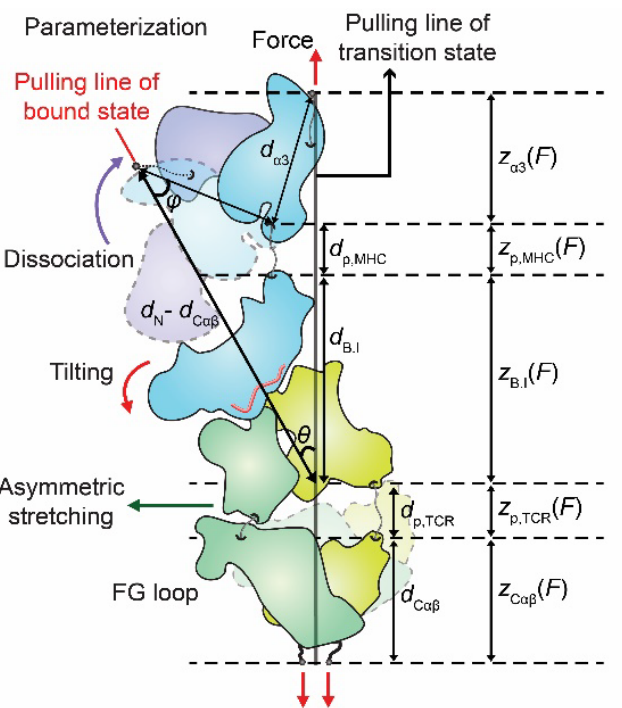

D
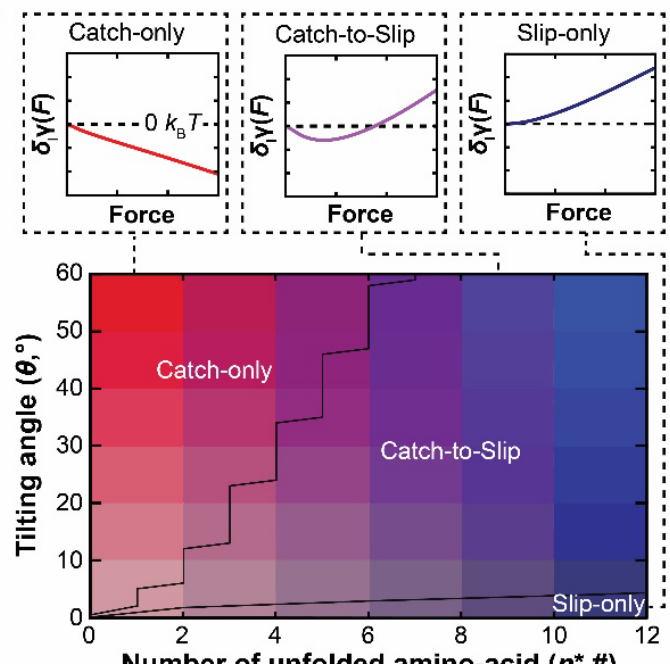

G
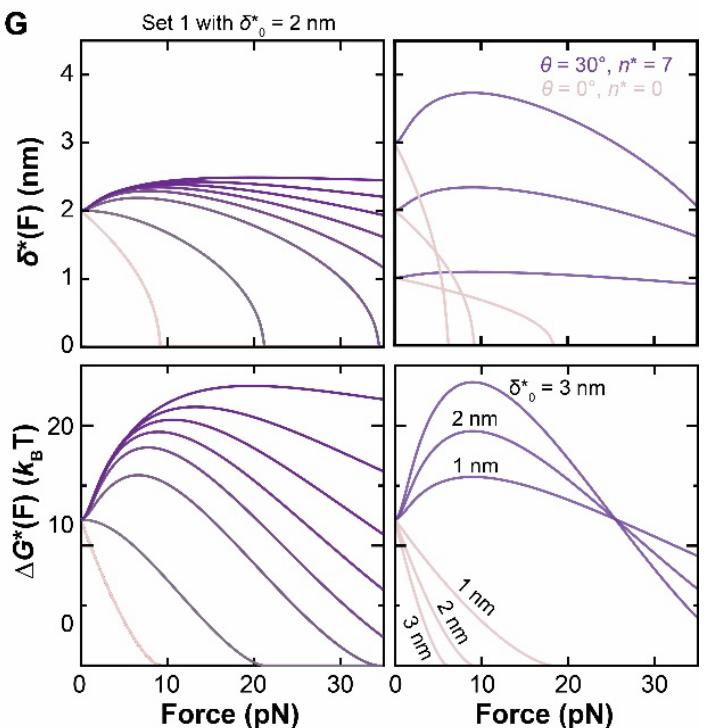
Figure 1. Structure, mechanics, and characteristics of the TCR-pMHC-I catch bond

model. (A) Force-induced conformational changes - partial unfolding, stretching, and rotation - of a TCR-pMHC-I complex as it traverses from the bound state to the transition state through the dissociation pathway through a 1D energy landscape. The diagrams of the $2 \mathrm{C}$ TCR $\alpha$ (yellow) $\beta$ (green) subunits and the DEVA peptide (red) bound to the $\mathrm{H} 2-\mathrm{K}^{\mathrm{b}}$ (various domains indicated) are based on snapshots from SMD simulations performed on the complex structure $(2 \mathrm{CKB})$ at the initial time (bound state) and a later time (transition state) (5). The forcetransmission path is shown as red lines connecting the force-acting nodes. (B) Breakdown of the total extension into various contributions projected onto the force axis: rotation of the $\alpha_{3}$ and $\beta_{2 m}$ domains about the MHC C-terminus upon dissociation of the $\beta_{2 m}-\alpha_{1} \alpha_{2}$ interdomain bond $\left(z_{\alpha 3}\right)$, relative rotation between $\alpha_{3}$ and $\alpha_{1} \alpha_{2}$ about their interdomain hinge that is also stretched $\left(z_{\mathrm{p}, \mathrm{MHC}}\right)$, tilting of the MHC $\alpha_{1} \alpha_{2}$ complexed with TCR $\operatorname{V} \alpha \beta\left(z_{\mathrm{B} . \mathrm{I}}\right)$, rotation about and extension of the $\mathrm{V} \alpha-\mathrm{V} \beta$ interdomain hinge $\left(z_{\mathrm{p}, \mathrm{TCR}}\right)$, and extension of the $\mathrm{C} \alpha \beta$ and rotation about their C-termini $\left(z_{C \alpha \beta}\right)$. Note that two $\alpha_{3}-\beta_{2 m}$ structures are shown: one before (light colors) and the other after (dark colors) $\beta_{2 m}$ dissociation from the $\alpha_{1} \alpha_{2}$ domain. Two structural parameters introduced to describe the above contributions to the total extension are shown: $d_{\alpha 3}$ is the distance between the $\alpha 3 \mathrm{C}$ - and $\mathrm{N}$-termini excluding the $\alpha_{1} \alpha_{2}-\alpha 3$ hinge. $\theta$ is the angle between the normal direction of the TCR-pMHC bonding interface at the bound state before conformational changes, which is vertical (cf. A, left), and that at the transition state after conformational changes, which is tilted (cf. A, right). (C) Extension change vs force curves (lower) for the color-matched $n^{*}$ and $\theta$ values (upper). The left panel (set 1 in upper table) shows the effect of changing $\theta$ with $\left(n^{*}=7\right)$ and without $\left(n^{*}=0\right)$ partial unfolding. The right panel (set 2) shows the effect of changing $n^{*}$ with $\left(\theta=30^{\circ}\right)$ and without $\left(\theta=0^{\circ}\right)$ rotation. (D) 
$n^{*}-\theta$ phase diagram showing three parameter domains: slip-only, catch-slip, and catch-only respectively colored by red-purple, purple-blue, and blue-black. Upper insets indicate representative energy change $\delta_{1} \gamma$ vs force curves for each bond type. (E) Theoretical normalized bond lifetime vs force curves for indicated parameters. The upper and lower panels show the respective effects of changing $\theta$ and $\delta_{0}^{*}$ from the set 1 parameters defined in C. (F) Energy landscapes expressed as families $\Delta G$ vs $\delta_{l}$ curves for a range of forces for slip-only (upper) and catch-slip (lower) bonds. The bound state is located at the origin $\Delta G=0$ and $\delta_{l}=$ 0 . The transition state has an energy of $\Delta G^{*}$ located at $\delta^{*}$ when $F>0$ and $\Delta G_{0}^{*}$ located at $\delta_{0}^{*}$ when $F=0$. (G) Plots of transition state location $\delta^{*}$ (upper) and height of energy barrier $\Delta G^{*}$ (lower) vs force $F$ for changing $\theta$ while keeping $\delta_{0}^{*}$ constant (left) or changing $\delta_{0}^{*}$ while keeping $\theta$ constant (right) for the indicated values from parameter table in C. 
A OT1 TCR (from CD8 ${ }^{+}$naïve T cell):peptide:H2-K $\mathrm{K}^{\mathrm{b}} \mathrm{aA} 2$

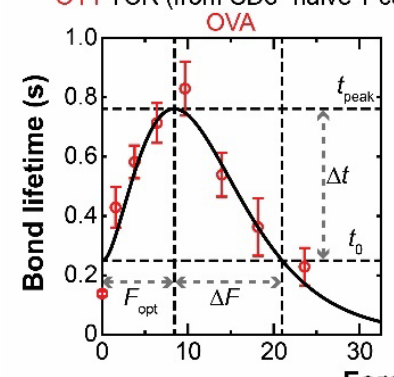

Force (pN)

C O OT1 TCR:p:H2-Kb(a3A2)

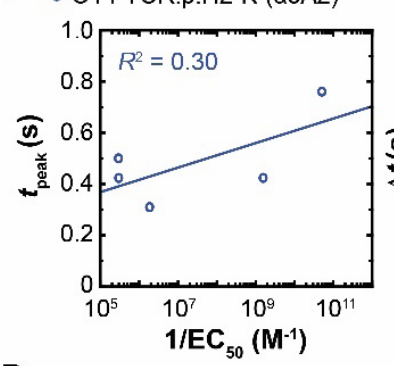

D

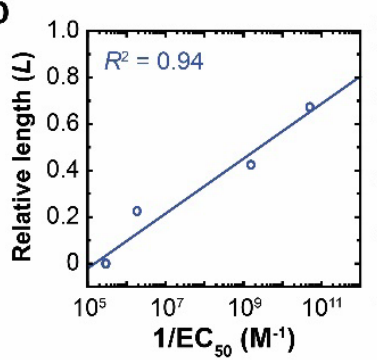

E
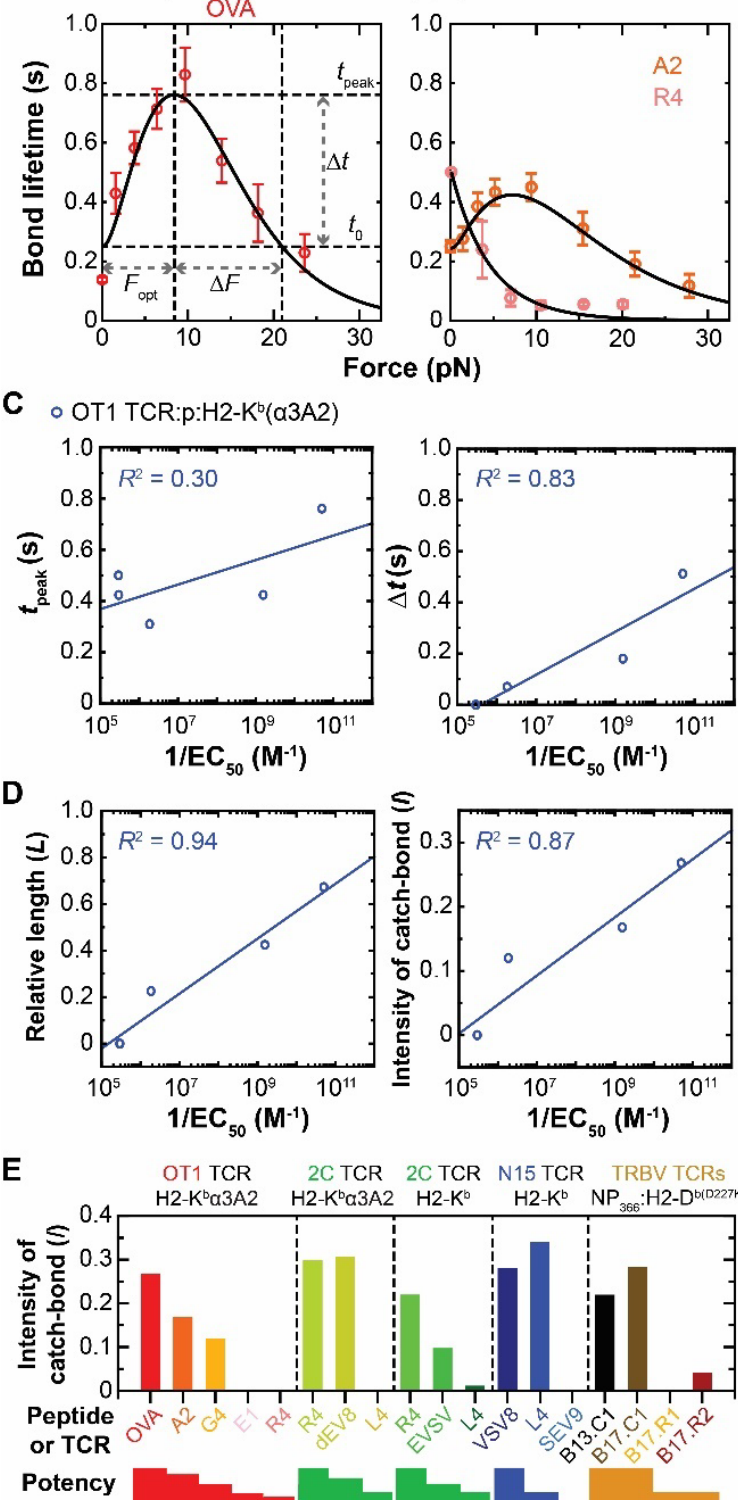

B

F
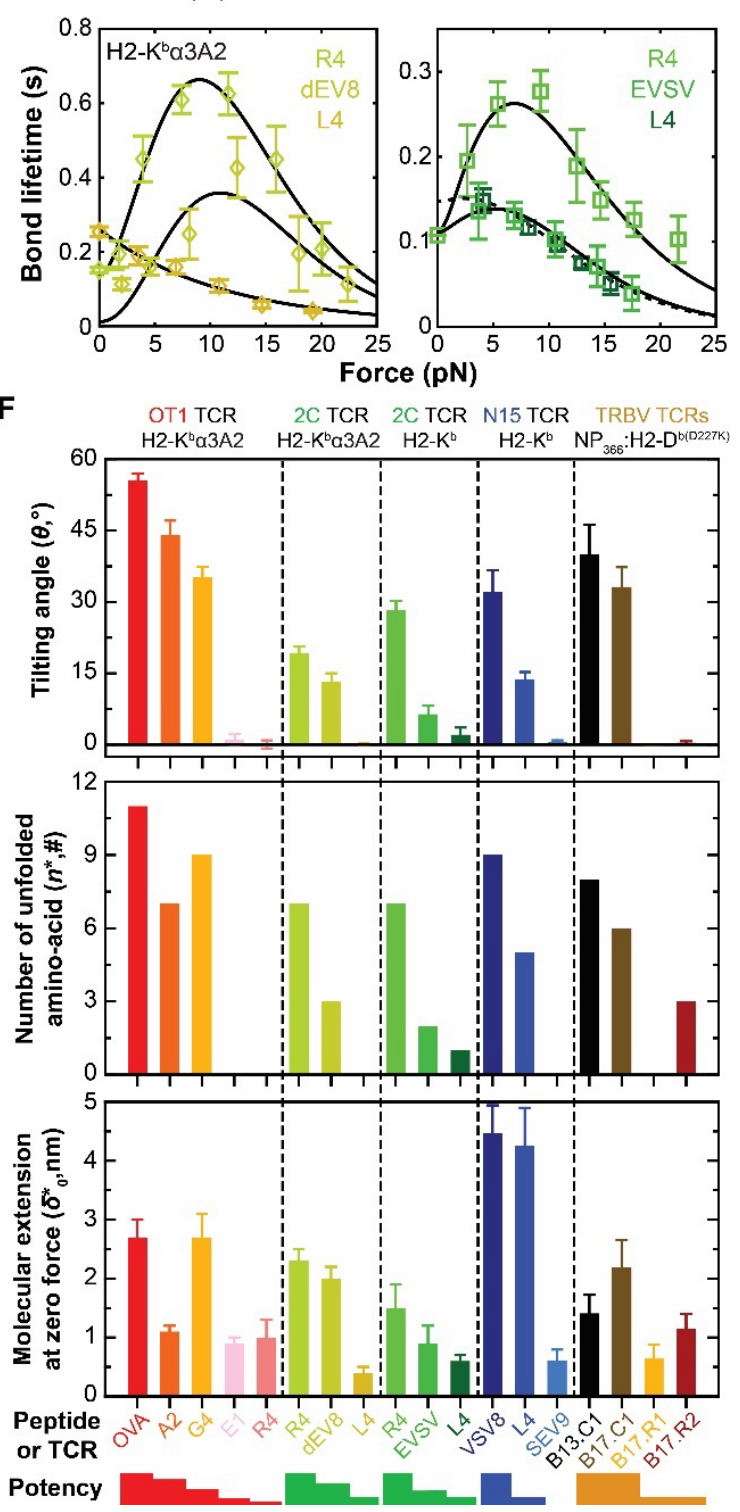

Figure 2. TCR bond type characterization and correlation with pMHC-I biological

activity. (A, B) Fitting of theoretical $1 / k(F)$ curves to experimental bond lifetime vs force data (points, mean \pm sem, re-analyzed from $(2,5))$ of OT1 (A) or $2 \mathrm{C}(\mathrm{B}) \mathrm{TCR}$ expressed on CD8 ${ }^{+}$ naïve T cells interacting with indicated $\mathrm{p}: \mathrm{H} 2-\mathrm{K}^{\mathrm{b}} \alpha 3 \mathrm{~A} 2$ (A and $\mathrm{B}$ left) or on $\mathrm{CD} 8^{-} 2 \mathrm{C}$ hybridomas with interacting with indicated $\mathrm{p}: \mathrm{H} 2-\mathrm{K}^{\mathrm{b}}(\mathrm{B}$, right $)$. Several metrics are defined to characterize the force-lifetime curve as indicated in the left panel of A: and $F_{\text {opt }}$ is the "optimal force" where lifetime peaks $\left(t_{\text {peak }}\right), \Delta t$ is the lifetime increase from the zero-force value $t_{0}$ to $t_{\text {peak }}$, and $\Delta F$ 
is the force increase from $F_{\text {opt }}$ to the force where lifetime returns to $t_{0}$. (C, D) Twodimensional metrics, $\boldsymbol{t}_{\text {peak }}$ and $\Delta \boldsymbol{t}(\mathrm{C})$, and two dimensionless metrics, $\boldsymbol{L}=\Delta t / t_{\text {peak }}$ and $\boldsymbol{I}=$ $L /(1+B)$ where $B=\Delta F / F_{\text {opt }}(\mathrm{D})$, was plotted vs the logarithm of the peptide concentration required to stimulate half-maximal $\mathrm{T}$ cell proliferation $\left(1 / \mathrm{EC}_{50}\right)$ and fitted by a linear function. (E) Intensity of catch bond, I calculated using the fitted force-lifetime curves of OT1, 2C TCR on primary $\mathrm{T}$ cells, 2C TCR on hybridomas, purified N15 TCR $\alpha \beta$, or TRBV TCRs (B13.C1/B17.C1 and B17.R1/B17.R2) expressed on hybridomas interacting with their corresponding pMHCs are plotted according to the ranked-order of peptide potencies (bottom). (F) Best-fit model parameters $\theta$ (the tilted angle of the bonding interface, upper), $n^{*}$ (the number of unfolded amino acids, middle), and $\delta_{0}^{*}$ (the width of zero-force free-energy well, lower) are plotted according to the ranked order of the peptide potencies (bottom). 
A

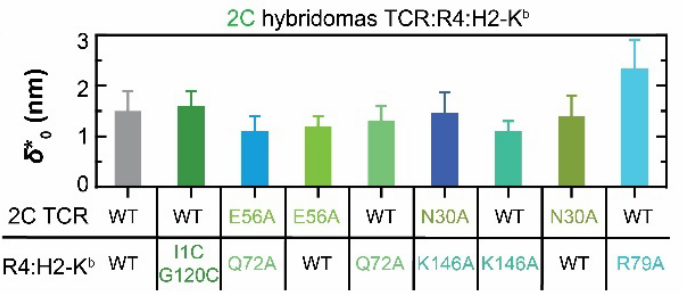

B

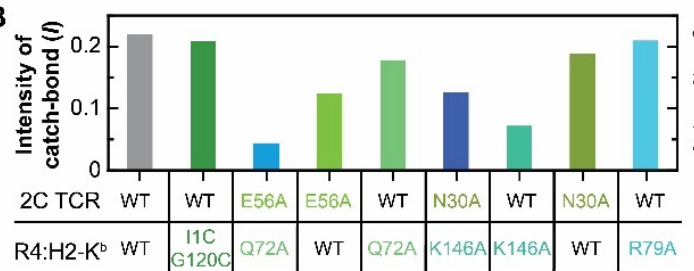

D

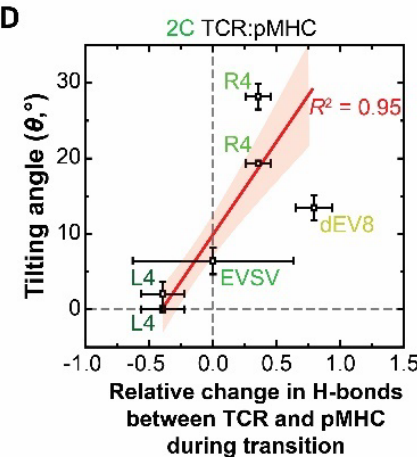

G

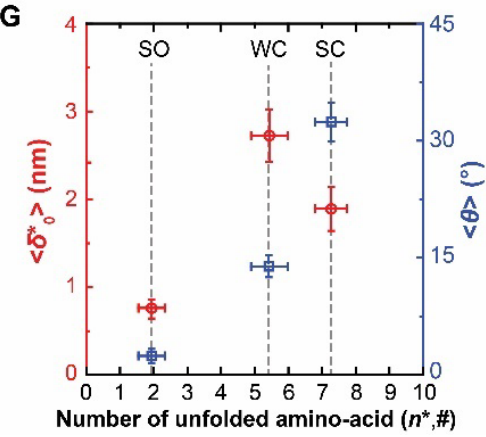

E

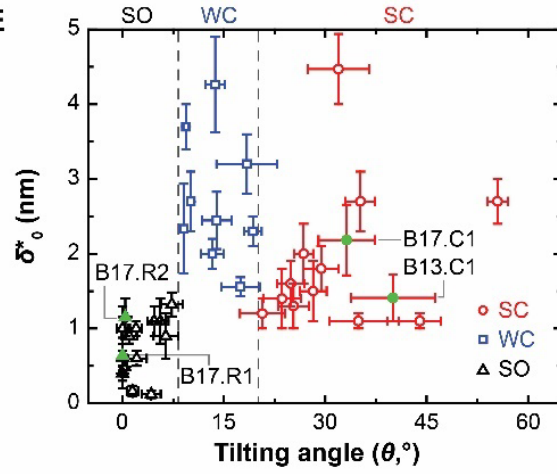

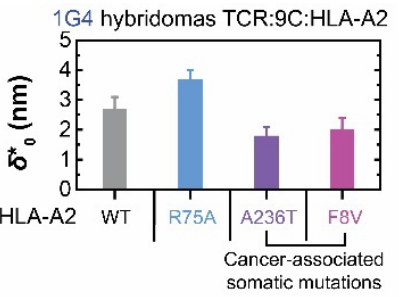

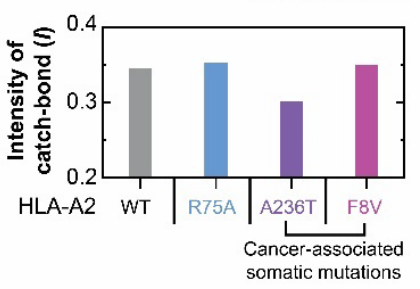

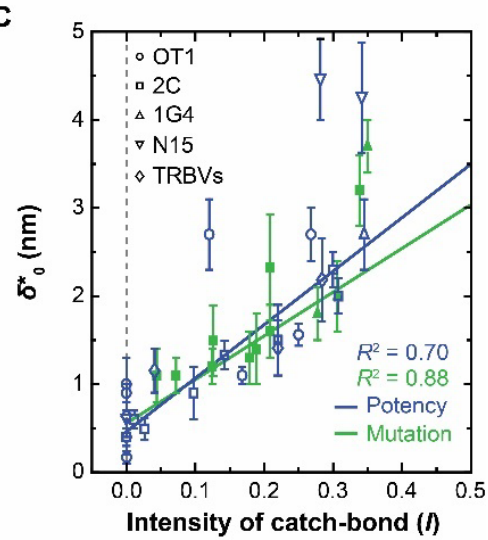
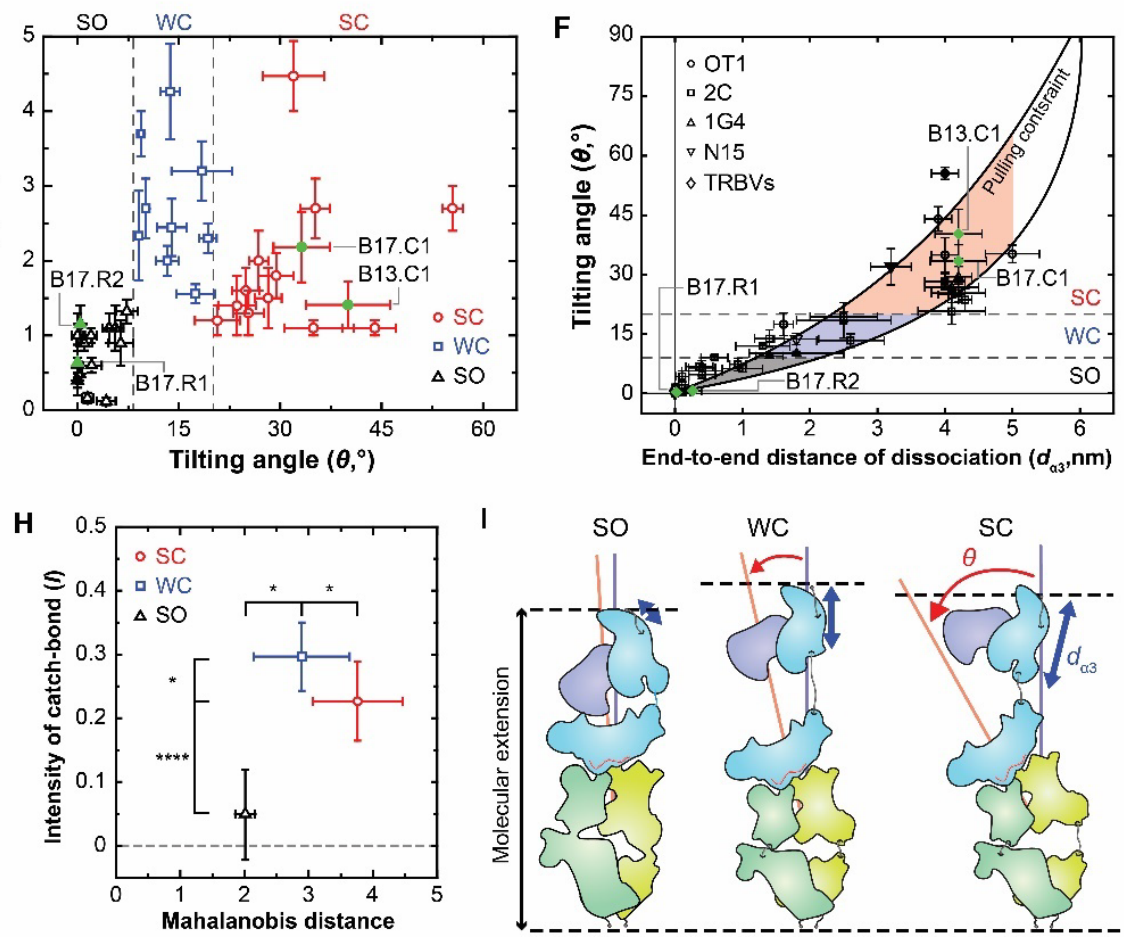

Figure 3. Properties and biological relevance of class I model parameters. (A, B) The width of zero-force energy well $\delta_{0}^{*}(\mathrm{~A})$ and the intensity of catch bond $I(\mathrm{~B})$ calculated from WT or mutant 2C TCRs (left) and WT 1G4 TCR (right) interacting with their corresponding WT or mutant pMHCs. The mutant $2 \mathrm{C}$ TCRs and $\mathrm{H} 2-\mathrm{K}^{\mathrm{b}} \mathrm{s}$ were designed to destabilize the TCR-MHC interaction. The mutant HLA-A2s were designed to either destabilize the TCR-pMHC interaction (R75A) or stabilize the MHC intramolecular interaction (A236T and F8V). (C) Data from Fig. $2 E$ and $F\left(3^{\text {rd }}\right.$ row) are re-graphed as $\delta_{0}^{*}$ vs $I$ plot to show their correlation (blue). Additional $\delta_{0}^{*}$ vs $I$ data from mutant TCRs and/or mutant pMHCs without functional data also 
show strong correlation (green). Different TCR systems are indicated by different symbols. The two datasets were separately fitted by two straight lines with the goodness-of-fit indicated by $R^{2}$. (D) Tilting angle of the bonding interface $(\theta)$ vs normalized net gain of hydrogen bonds at the interface between 2C TCR and the indicated pMHCs is plotted (points) and fitted (line), with $95 \%$ confident interval shown as shaded area. (E) Clustering analysis reveals three clusters in the $\delta_{0}^{*}-\theta$ phase diagram: slip-only (SO, black), weak catch-slip (WC, blue), and strong catch-slip (SC, red) bonds. Closed-symbols represent bonds of the WT TCR with WT agonist peptides. (F) Tilting angle $(\theta)$ vs end-to-end distance of dissociated $\alpha 3$ domain $\left(d_{\alpha 3}\right)$. The three types of bonds, SO, WC, and SC, are also clustered in this phase diagram, which are separated by the dotted lines that predicted from the pulling constraints of the model. The two pairs of TRBV TCRs are indicated in E and F by green dots. (G) The average molecular extensions at zero force $\left(\left\langle\delta_{0}^{*}\right\rangle\right.$, left ordinate) and the average rotation angle $(\langle\theta\rangle$, right ordinate) are plotted vs the total number of unfolded amino acids ( $n^{*}$, abscissa) to show three clusters. Each bond type is indicated by a dotted line. (H) Intensity of bond type vs Mahalanobis distance plot, again showing three clusters. Principal component analysis was used to find principal axes. Mahalanobis distances for each cluster were calculated using common principal axes from total data set. (I) Cartoons illustrating the conformations of three bond types based on their model parameters. Two structural parameters $\left(\theta\right.$, red; $d_{\alpha 3}$, blue $)$ are shown to exemplify the difference between bond types. All error bars are either fitting errors or statistically propagated errors from fitting results. 
A
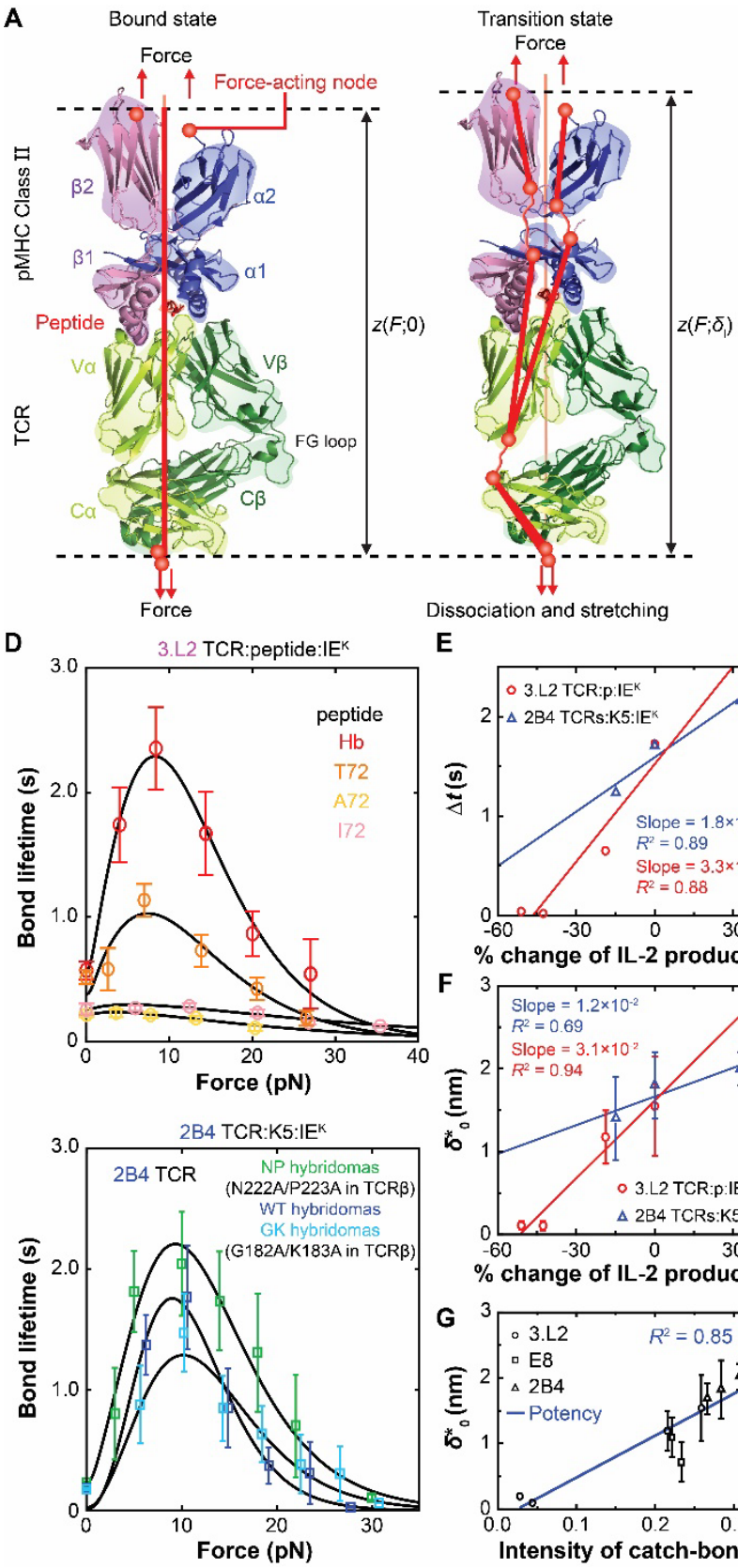
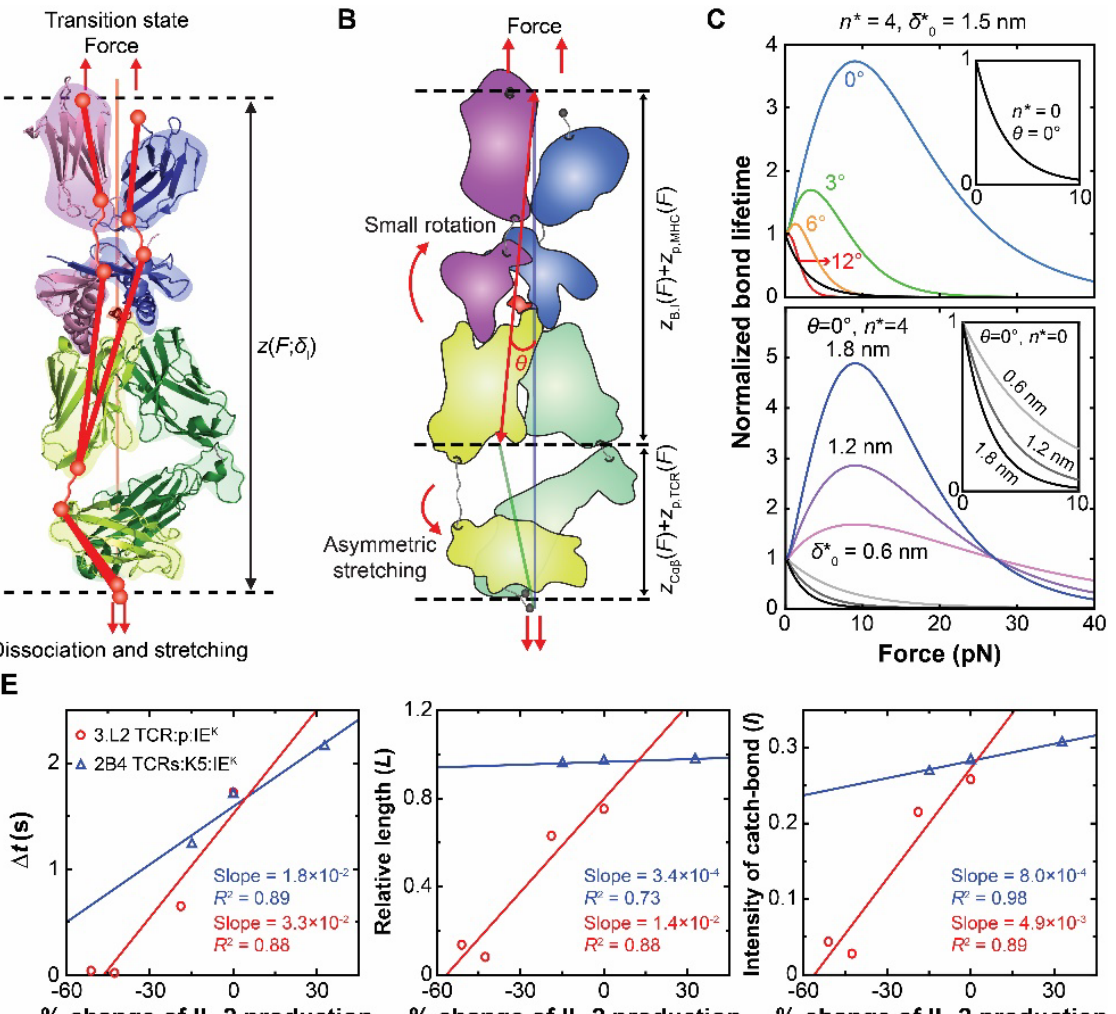

F 3 change of IL-2 production
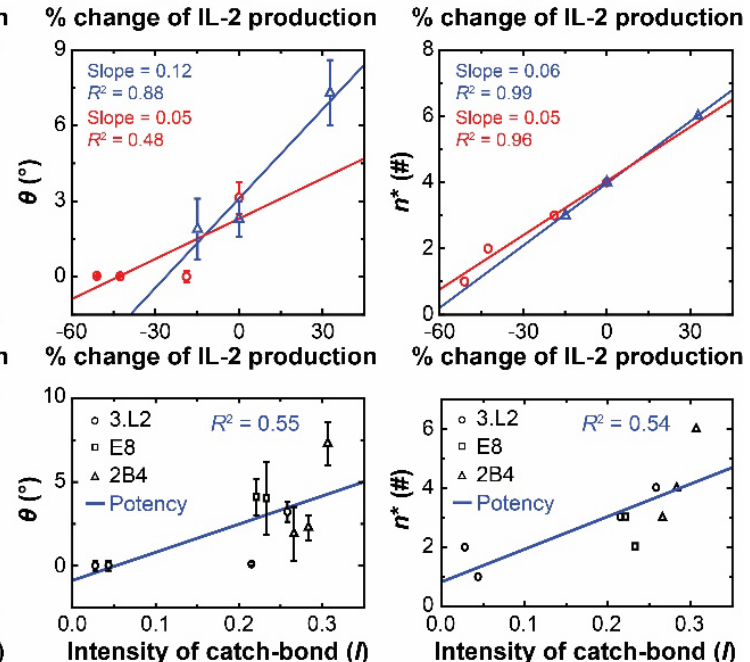

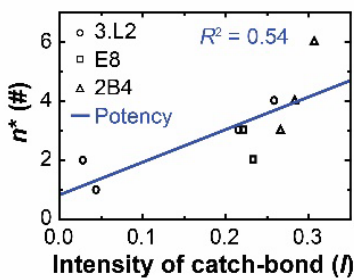

Figure 4. The TCR-pMHC-II model. (A) Force-induced conformational changes - partial unfolding, stretching, and rotation - of a TCR-pMHC-II complex as it traverses from the bound state to the transition state through the dissociation pathway through a $1 \mathrm{D}$ energy landscape. The diagrams are based on the published co-crystal structure (2IAM) of the E8 TCR $\alpha$ (yellow) $\beta$ (green) subunits and the TPI peptide (red) bound to the HLA-DR1 $\alpha$ (blue) $\beta$ (pink) subunits with various domains indicated. The force-transmission paths are shown as red lines connecting 
the force-acting nodes. (B) Breakdown of the total extension into various contributions projected onto the force axis: stretching of the TCR $\mathrm{C} \alpha$ and $\mathrm{C} \beta$ domains $\left(z_{\mathrm{C} \alpha \beta}\right)$, asymmetric partial unfolding of the TCR $\mathrm{V} \alpha-\mathrm{C} \alpha$ and $\mathrm{V} \beta-\mathrm{C} \beta$ interdomain hinges $\left(z_{\mathrm{p}, \mathrm{TCR}}\right)$, asymmetric partial unfolding of the MHC $\alpha 1-\alpha 2$ and $\beta 1-\beta 2$ interdomain hinges $\left(z_{\mathrm{p}, \mathrm{MHC}}\right)$, and small rotation between the $\alpha 1-\beta 1$ and $\alpha 2-\beta 2$ domain hinges and tilting of the bonding interface between the MHC $\alpha 1-\beta 1$ and the TCR V $\alpha-\mathrm{V} \beta$ by an angle $\theta\left(z_{\mathrm{B} . \mathrm{I}}\right)$. (C) Theoretical normalized bond lifetime vs force curves. The effects of changing $\theta$ and $\delta_{0}^{*}$ are shown in the upper and lower panels, respectively, for the indicated parameter values. (D) Fitting of class II model-predicted $1 / k(F)$ curves to experimental bond lifetime vs force data (points, mean \pm sem) of 3.L2 TCR on $\mathrm{CD}^{-} \mathrm{CD}^{+}$naive T cells interacting with indicated $\mathrm{p}: \mathrm{I}^{\mathrm{k}} \mathrm{E}^{\mathrm{k}}$ 's (3) (upper) or WT and indicated mutant 2B4 TCRs on hybridomas interacting with K5:I-E ${ }^{\mathrm{k}}(26)$. (E) Dimensional metrics, $\Delta \boldsymbol{t}$ (left), scaled relative length of bond lifetime $\boldsymbol{L}$ (middle), and intensity of catch bond $\boldsymbol{I}$ (right) vs \% change (relative to WT) in the peptide dose required to achieve half-maximum hybridoma IL-2 production $\left(1 / \mathrm{EC}_{50}\right)(27)(3 . \mathrm{L} 2$, red $)$ or in the area under the dose response curve (AUC) (28) (2B4, blue) plots. (F) Best-fit model parameters $\delta_{0}^{*}\left(\right.$ left), $\theta$ (middle), and $n^{*}($ right $)$ are plotted vs. relative \% change of hybridoma IL-2 production. (G) The three model parameters in $\mathrm{G}$ for both the 3.L2 and 2B4 TCR systems are plotted vs the catch-bond intensity $I$ and fitted by a straight line. We also added to each panel an additional point obtained from data and model fit of E8 TCR-TPI:HLA-DR1 interactions (52). 

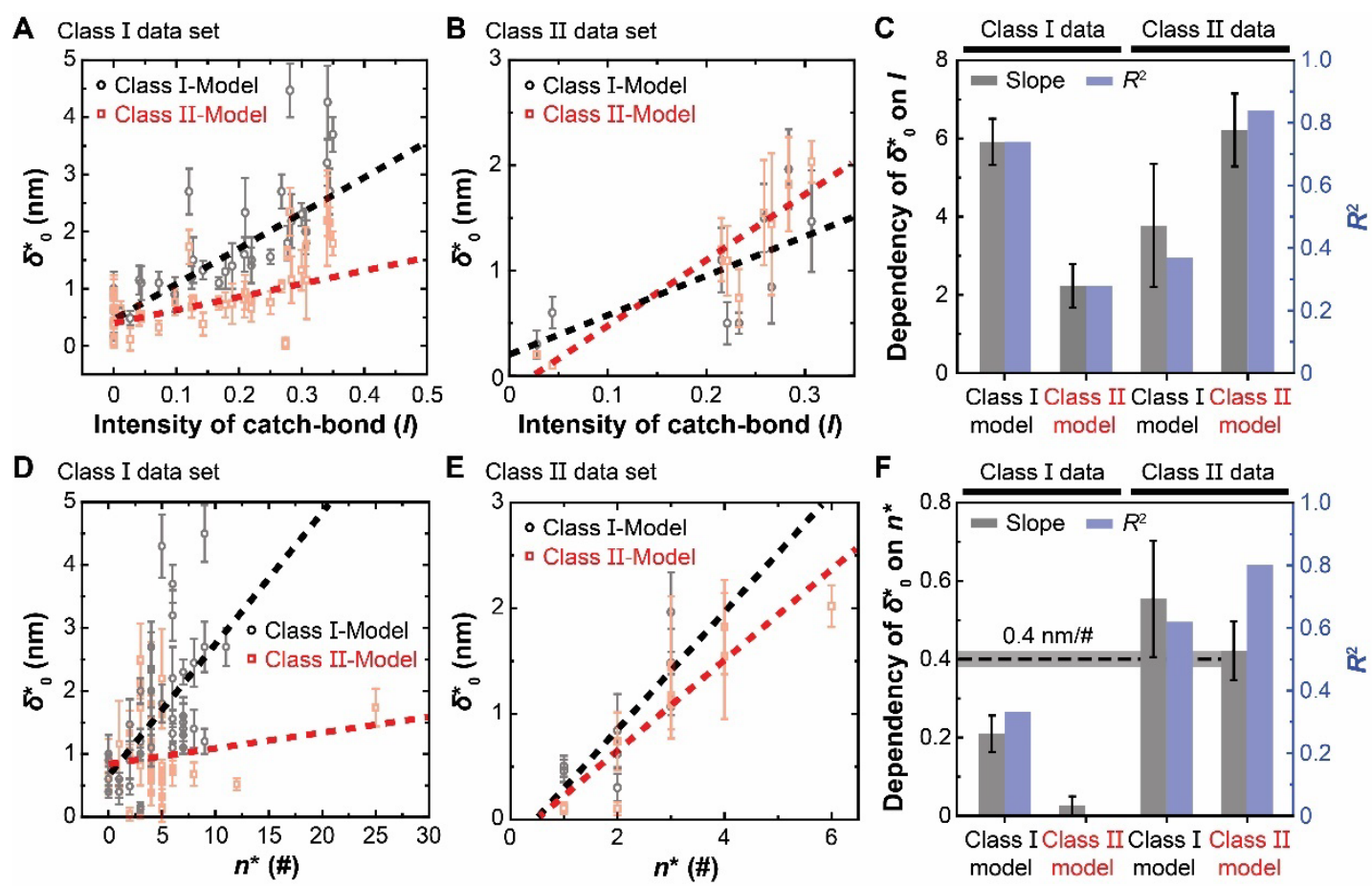

Figure 5. Cross-examination of class I and II models against class I and II data. (A, B)

$\delta_{0}^{*}$ vs I plots obtained using class I (black) or class II (red) model to fit bond lifetime vs force data of TCR interacting with pMHC-I (A) or pMHC-II (B) molecules. In each panel, two sets of parameter values were returned from fitting depending on whether class I (black) or class II (red) model was used because they are based on different structures and conformational changes of the TCR-pMHC complexes. (C) The slopes (gray bar, the level of correlation between $\delta_{0}^{*}$ and $I$ ) and goodness-of-fit $\left(R^{2}\right)$ (blue bar, the degree of appropriateness of the model for the data) of the linear fit in A and B are shown in the matched $\left(1^{\text {st }}\right.$ and $4^{\text {th }}$ groups) and mismatched $\left(2^{\text {nd }}\right.$ and $3^{\text {rd }}$ groups $)$ cases. $(\mathbf{D}, \mathbf{E}) \delta_{0}^{*}$ vs $n^{*}$ plots obtained using class I (black) or class II (red) model to fit bond lifetime vs force data of TCR interacting with pMHC-I (D) or pMHC-II (E) molecules. (F) The slopes and goodness-of-fit of the linear fit in $\mathrm{D}$ and $\mathrm{E}$ are shown in the matched $\left(1^{\text {st }}\right.$ and $4^{\text {th }}$ groups $)$ and mismatched $\left(2^{\text {nd }}\right.$ and $3^{\text {rd }}$ groups $)$ cases. The slopes indicate the average unfolding extension per amino acid (nm/a.a) from each 
model, which are compared to the maximum average contour length per amino acid of $\sim 0.4$

nm/a.a. (biophysical limit, black dashed line with considerable deviation) $(17,29,30)$. 


\title{
Supplementary Materials
}

\section{Supplementary Materials for}

\section{Catch bond models explain how force amplifies TCR signaling and antigen discrimination}

\author{
Hyun-Kyu Choi, Kaitao Li, Muaz Nik Rushdi, Chenghao Ge, Wei Chen, Jizhong Lou, and \\ Cheng Zhu* \\ *Corresponding author. Email: cheng.zhu@bme.gatech.edu
}

This PDF file includes:

Supplemental Model Derivations

Figs. S1 to S10

Tables S1 to S5

References (56 to 62) 


\section{Table of Contents}

\section{Supplemental Model Derivations}

A. Kinetic model for TCR-pMHC-I

A.1. Derivation of the force-induced energy change $\delta_{1} \gamma(F)$

A.2. Simplifying assumptions and reducing parameters

A.3. Defining the dissociation coordinate

A.4. Model applications, curve-fitting strategies, and biological relevance

A.5. Class I model constraints

B. Kinetic model for TCR-pMHC-II

B.1. Development, validation, and characterization

B.2. Class II model constraints

C. A general biophysical limit of model parameters

\section{Supplemental Figures}

Fig. S1. Fitting class I model to data of TCR-pMHC-I bond lifetime vs force

Fig. S2. Correlation of class I model parameters with biological activity and TCR bond type

Fig. S3. Hydrogen $(\mathrm{H})$ bond distributions at the TCR-pMHC-I bonding interface

Fig. S4. Pulling and tilting constraints of class I model.

Fig. S5. Determining the number of clusters in parameter space

Fig. S6. Characterization of the energy landscape of TCR-pMHC-II dissociation

Fig. S7. Correlation of metrics of TCR-pMHC-II bond lifetime vs. force curves with T cell biological activity

Fig. S8. Comparing fitting curves of class I and class II models for the same data

Fig. S9. Relative goodness-of-fit of class I and II models to class I and class II data

Fig. S10. Comparison of pMHC interactions with cell surface and purified TCRs

\section{Supplemental Tables}

Table S1. Summary of Model Constants.

Table S2. Finding the Best-fit Parameters for OT1 TCR-OVA:H2-K ${ }^{\mathrm{b}} \mathrm{a}$ A2 Bond.

Table S3. Best-fitting Parameters for the TCR-pMHC-I Bonds.

Table S4. Finding the Best-fit Parameters for the 3.L2 TCR-Hb:I-E ${ }^{\mathrm{k}}$ Bond.

Table S5. Best-fit parameters for TCR-pMHC-II Bonds. 


\section{Supplemental Model Derivations}

\section{A. Kinetic model for TCR-pMHC-I}

\section{A.1. Derivation of the force-induced energy change $\delta_{1} \gamma(F)$}

The purpose of this subsection is to provide detailed derivation of the force-induced change in the energy landscape, or the work done by force as the TCR-pMHC complex is stretched at the two ends from the bound state to the transition state until dissociation (Fig. $1 \mathrm{~A}$ and $B$ ). Mathematically, $\delta_{1} \gamma(F)$ is an integral over a force range $0 \leq f \leq F$, where $F$ is the level of force under which the kinetic rate is being evaluated (Eq. 2). The integrand $\delta_{\mathrm{z}}(f)$ is the projection on the force direction of the length change of the TCR-pMHC structure relative to its bound state length during dissociation. Subtraction of this work from the interaction free energy tilts the energy landscape that governs the off-rate (Eq. 1). To calculate molecular stretch, we assume the TCR-pMHC complex to behave as a system of semi-rigid bodies of globular domains connected by semi-flexible polymers (Fig. 1B). As such, the total length change includes three components: 1) extension of individual globular domains, 2) various domain rotations about hinges, and 3) unfolding of secondary structures at specific regions.

For a globular domain without unfolding, the force-extension relationship is described by the three-dimensional freely-jointed chain model (22):

$$
d_{\mathrm{i}}(f)=d_{\mathrm{i}, \mathrm{c}}\left\{\operatorname{coth}\left[f d_{\mathrm{i}, \mathrm{c}} / k_{\mathrm{B}} T\right]-\frac{k_{\mathrm{B}} T}{f d_{\mathrm{i}, \mathrm{c}}}\right\}\left(1+\frac{f}{E_{\mathrm{d}}}\right),
$$

where $d_{\mathrm{i}}$ is end-to-end distance of the $i$ th-domain, $k_{\mathrm{B}}$ is the Boltzmann constant, $T$ is absolute temperature, $f$ is force, and $E_{\mathrm{d}} \sim 100 \mathrm{pN}$ is the elastic modulus of the folded globular domain (56). The present work considers domain extension of the whole TCR-pMHC-I complex $\left(d_{\mathrm{N}}\right)$ and three of its parts: the MHC $\alpha_{3}$ domain $\left(d_{\alpha 3}\right)$, bonding interface that includes the MHC $\alpha_{1} \alpha_{2}$ domains bound to the TCR V $\alpha \beta$ domains $\left(d_{\mathrm{B} . \mathrm{I}}\right)$, and the TCR C $\alpha \beta$ domains $\left(d_{\mathrm{C} \alpha \beta}\right)$. Their 
contour lengths, $d_{\mathrm{i}, \mathrm{c}}$, have well-defined values depending on the TCR-pMHC complex in question, which are summarized in Table S1. For example, $d_{\mathrm{N}, \mathrm{c}}=12.7$ and $10.9 \mathrm{~nm}$ for $2 \mathrm{C}$ TCR complexed with H2-K ${ }^{\mathrm{b}}$ (PDB codes 2CKB, 1MWA, and 1G6R) and H2-L ${ }^{\mathrm{d}} \mathrm{m} 31$ (2E7L), respectively, and $12.3 \mathrm{~nm}$ for $1 \mathrm{G} 4 \mathrm{TCR}$ complexed with HLA-A2 (2BNR and 2BNQ). We also choose $11.6 \mathrm{~nm}$ as a reasonable guess value for the OT1 TCR $-\mathrm{p}: \mathrm{H} 2-\mathrm{K}^{\mathrm{b}}$ complex.

To calculate the work $\delta_{1} \gamma(F)$, we project the above domain extensions onto the direction of force, which is taken as the $z$ direction (Fig. 1B) using two angles between the $z$ axis and: 1) the normal direction of the bonding interface $(\theta)$ and 2) the line connecting the $\mathrm{C}$ and N-termini of the MHC-I $\alpha_{3}$ domain excluding any unfolded residues $(\varphi)$ :

$$
\begin{gathered}
z_{\mathrm{N}}(f)=d_{\mathrm{N}}(f) \\
z_{\alpha 3}(f)=\sqrt{d_{\alpha 3}^{2}(f)-\left\{\left[d_{\mathrm{N}}(f)-d_{\mathrm{C} \alpha \beta}(f)\right] \sin \theta-d_{\alpha 3}(f) \sin (\varphi+\theta)\right\}^{2}}, \\
z_{\mathrm{B} . \mathrm{I}}(f)=d_{\mathrm{B} . \mathrm{I}}(f) \equiv\left[d_{\mathrm{N}}(f)-d_{\mathrm{C} \alpha \beta}(f)\right] \cos \theta-d_{\alpha 3}(f) \cos (\varphi+\theta), \\
z_{\mathrm{C} \alpha \beta}(f)=d_{\mathrm{C} \alpha \beta}(f) .
\end{gathered}
$$

Note that $\theta$ is a model parameter as it describes the tilting of the bonding interface as part of the force-induced conformational change, whereas $\varphi$ is a model constant is measured from the crystal structure (Table S1, see A.2 below).

We assume that partial unfolding in the molecular complex may occur at connecting regions of globular domains. In particular, the $\alpha_{1} \alpha_{2}-\alpha_{3}$ joint of the MHC-I and the V $\alpha-C \alpha$ interdomain joint of the TCR (Fig. 1A). The former may be caused by dissociation of the noncovalent $\alpha_{1} \alpha_{2}-\beta_{2 m}$ interdomain bond, which shifts the mechanical load originally borne by this bond to the $\alpha_{1} \alpha_{2}-\alpha_{3}$ hinge, resulting in its partial unfolding, as observed in SMD simulations (5) (Fig. 1A). Similarly, $\alpha_{1} \alpha_{2}-\beta_{2 \mathrm{~m}}$ dissociation results in tilting of the bonding interface and load shifting from the $\mathrm{V} \beta-\mathrm{C} \beta$ joint to the $\mathrm{V} \alpha-\mathrm{C} \alpha$ joint, leading to partial 
unfolding of the latter joint (Fig. 1A). The unfolded polypeptides are flexible and can bear only tension but not moment, ensuring that their extension is along the direction of force, i.e., the $z$ axis.

The force-extension relationship of the unfolded polypeptides can be described by an extensible worm-like chain (eWLC) model (23):

$$
\frac{f l_{\mathrm{p}}}{k_{\mathrm{B}} T}=\frac{1}{4\left(1-z_{\mathrm{p}} /\left(n l_{\mathrm{c}}\right)\right)}-\frac{1}{4}+\frac{z_{\mathrm{p}}}{n l_{\mathrm{c}}}-\frac{f}{K_{\mathrm{p}}}+\sum_{\mathrm{j}=1}^{\mathrm{j} \leq 7} a_{\mathrm{j}}\left(\frac{z_{\mathrm{p}}}{n l_{\mathrm{c}}}-\frac{f}{E_{\mathrm{p}}}\right)^{\mathrm{j}}
$$

where $z_{\mathrm{p}}$ is the extension of the unfolded coil under force with the subscript $p$ indicating unstructured polypeptide, $l_{\mathrm{c}}=0.36 \mathrm{~nm}$ and $l_{\mathrm{p}}=0.39 \mathrm{~nm}$ are the average contour length and persistence length per unfolded amino acid, respectively $(29,57,58), E_{\mathrm{p}} \sim 50 \mu \mathrm{N}$ is the elastic modulus of polypeptides (57), $a_{\mathrm{j}}$ are polynomial coefficients for the improved approximation, and $n$ is the number of constituent amino acids in the unfolded polypeptide. In particular, we denote the respective numbers of amino acids in the unfolded MHC-I $\alpha_{1} \alpha_{2}-\alpha_{3}$ and TCR V $\alpha-$ C $\alpha$ joints to be $n_{\mathrm{p}, \mathrm{MHC}}$ and $n_{\mathrm{p}, \mathrm{TCR}}$. Eq. S3 defines $z_{\mathrm{p}}$ as a function of $f$, which can be solved by numerical computation to express in an explicit form: $z_{\mathrm{p}} / n l_{\mathrm{c}}=z_{\mathrm{u}, \mathrm{p}}(f)=$ the extension per unit contour length for the polypeptide under force $f$.

Thus, the length of the TCR-pMHC complex at the transition state is (Fig. 1B):

$$
z(f)=z_{\alpha 3}(f)+n_{\mathrm{p}, \mathrm{MHC}} l_{\mathrm{c}} z_{\mathrm{u}, \mathrm{p}}(f)+z_{\mathrm{B} . \mathrm{I}}(f)+n_{\mathrm{p}, \mathrm{TCR}} l_{\mathrm{c}} z_{\mathrm{u}, \mathrm{p}}(f)+z_{\mathrm{C} \alpha \beta}(f)
$$

Since we do not have prior knowledge about either number of unfolded amino acids, we will evaluate their sum $n^{*}=n_{\mathrm{p}, \mathrm{TCR}}+n_{\mathrm{p}, \mathrm{MHC}}$ from curve-fitting of our model to the experimental data (see below). Since $d_{\mathrm{N}}(f)$ is the length of the TCR-pMHC complex at the bound state (Fig. $1 B)$, we have $z(f ; 0)=d_{\mathrm{N}}(f)$. Finally, the integrand on the right-hand side of Eq. 3 can be written as 


$$
\delta_{\mathrm{z}}(f)=z_{\alpha 3}(f)+z_{\mathrm{B} . \mathrm{I}}(f)+z_{\mathrm{C} \alpha \beta}(f)+n^{*} l_{\mathrm{c}} z_{\mathrm{u}, \mathrm{p}}(f)-d_{\mathrm{N}}(f) .
$$

\section{A.2. Simplifying assumptions and reducing parameters.}

The purpose of this subsection is to provide the details omitted in the main text of how the model parameters are reduced to the smallest possible set and the underlying simplifying assumptions. To begin, we take the $\varphi$ values from PDB data for the specific TCR-pMHC interactions in question (Table S1). Due to their small range $\left(5^{\circ} \leq \varphi \leq 23.5^{\circ}\right)$, we assume that the $\varphi$ value remains constant during forced dissociation. Additionally, due to the semi-rigid approximation, the thickness of constant domain of TCR $\alpha$ - and $\beta$-subunit $\left(d_{\mathrm{C} \alpha \beta}\right)$ was also treated as constant value for each construct (Table S1). This leaves only two structural parameters $\left(d_{\alpha 3}, \theta\right)$ in our model to be evaluated by curve-fitting to data. Using the fact that the structure-based force function $\gamma\left(F ; \delta_{\mathrm{l}}\right)$ scales with the characteristic extension change per unit change of molecular length such that $\delta_{\mathrm{l}} \gamma(F)=\int_{0}^{F} \delta_{\mathrm{z}}(f) d f$, the dissociation rate of TCRpMHC-I bond can be written as follows:

$$
k(F)=k_{0} \sqrt{1-\frac{2 \int_{0}^{F} \delta_{\mathrm{z}}(f) d f}{3 \Delta G_{0}^{*}}} e^{\frac{\Delta G_{0}^{*}}{k_{\mathrm{B}} T}\left(1-\left(1-\frac{2 \int_{0}^{F} \delta_{\mathrm{Z}}(f) d f}{3 \Delta G_{0}^{*}}\right)^{3 / 2}\right)}
$$

where $\delta_{\mathrm{z}}(f)$ is given by Eq. S5.

To further reduce model parameters, we make additional assumptions as discussed in the main text. It is well-known that the fractions of free-energy change in biological interactions in liquid, such as unfolding and refolding of proteins, unbinding and rebinding of receptorligand bonds, and unzipping and rezipping of RNA or DNA, are small because of their limited dynamic transition time (59-62). Such a speed limit results from the nature of biological interactions, e.g., polar/non-polar interactions, hydrophobic interactions, and charged interactions, which typically yield finite range of transition kinetics, enabling us to roughly 
estimate the free-energy barrier as $\Delta G_{0}^{*} \sim \ln \left(k_{\mathrm{w}} / k_{0}\right)$ where $k_{\mathrm{w}} \sim 10^{6} \mathrm{~s}^{-1}$ known as the prefactor (59-62).

\section{A.3. Defining the dissociation coordinate.}

The purpose of this subsection is to derive an operational way to determine the dissociation coordinate variable $\delta_{1}$ used in the main text. We note that the total number of unfolded amino acids $n^{*}$ is zero at the bound state before unfolding occurs, increases during progressive unfolding along the dissociation path, and reaches maximum at the dissociation point. Because $n^{*}$ is not known a priori, it must be treated as a fitting parameter similar to $d_{\alpha 3}$, $\theta$, and $\delta_{0}^{*}$. Since $\delta_{\mathrm{l}}$ is the contour length change along the dissociation path, we wish that $\delta_{\mathrm{l}}$ approaches its upper bound $\delta_{0}^{*}$ and depends on force implicitly through the model parameters $n^{*}, d_{\alpha 3}$, and $\theta$, as given below:

$$
\delta_{\mathrm{l}}=n^{*} l_{\mathrm{c}}+\Delta\left(d_{\alpha 3}, \theta\right) \rightarrow \delta_{0}^{*},
$$

where $\Delta\left(d_{\alpha 3}, \theta\right)$ is the difference of the contour lengths except for the partially unfolded regions. Thus, Eq. S7 provides a constraint for $\delta_{\mathrm{l}}$ instead of introducing another model parameter. Since $d_{\alpha 3}$ and $\theta$ are determined for each $n^{*}$ during the model fitting that search for parameters such enable $\delta_{1} \rightarrow \delta_{0}^{*}$, small errors may occur for the various length components in $\Delta$ (red lines labeled as force transmission lines in Fig. $1 A$ ) but can be identified using a pair of $\left(d_{\alpha 3}, \theta\right)$ values and the crystal structure for each complex. Specifically, average differences $\langle\Delta\rangle$ are $-0.9 \mathrm{~nm}$ for the strong catch bond (SC) group $\left(d_{\alpha 3}>3 \mathrm{~nm}, \theta>20^{\circ}\right), 0.4 \mathrm{~nm}$ for the weak catch bond (WC) group $\left(1 \mathrm{~nm}<d_{\alpha 3}<3 \mathrm{~nm}, 5^{\circ}<\theta<20^{\circ}\right)$, and $0 \mathrm{~nm}$ for slip-only bond (SO) group ( $d_{\alpha 3}<1 \mathrm{~nm}, \theta<5^{\circ}$ ), respectively (see Fig. $3 E-I$ and associated text for the definitions of SC, WC, and SO groups). It has been well established that the contour length of a single amino acid is $\sim 0.4 \pm 0.02 \mathrm{~nm} / \mathrm{a} . \mathrm{a}(17,29,30)$, implying that the model has 3 amino acids resolution. We further note that, even without conformational change, it is possible for 
the slip-only bond group to have 3 unfolded amino acids due to the limited resolution of the model. Finally, the best-fit model parameters can be determined by finding the subset of bestfit parameter values among possible $n^{*}$ values that match the contour length change $\delta_{1}$ to the free-energy well width at zero-force $\delta_{0}^{*}$, i.e., finding $n^{*}$ such that $\delta_{1}\left(n^{*}, d_{\alpha 3}, \theta\right) \rightarrow \delta_{0}^{*}$ (see Table S2). Under this condition, $\int_{0}^{F} \delta_{\mathrm{z}}(f) d f=\delta_{\mathrm{l}} \gamma(F) \rightarrow \delta_{0}^{*} \gamma(F)$ and Eq. S6 becomes identical to Eq. 2.

\section{A.4. Model applications, curve-fitting strategies, and biological relevance}

The purpose of this subsection is to outline the procedures of applying our model to experiments. Our procedures include four steps: 1) examine how the model parameters control the model behaviors, 2) fit the model predicted reciprocal off-rate (Eq. S6) to the experimental bond lifetime vs force data, 3) construct the energy landscape and investigate its properties based on the parameters evaluated in part 2,4) elucidate the biological relevance of the model parameters.

The curve-fitting strategies involve varying one parameter while keeping others constant. For example, to investigate the effect of varying the titling angle $\theta$, we kept the other parameters constants (i.e., $n^{*}=7$, and $\delta_{0}^{*}=2 \mathrm{~nm}, k_{0}=3 \mathrm{~s}^{-1}$ and $\Delta G_{0}^{*}=12.7 k_{\mathrm{B}} T$ ), Also kept constant were several structural constants $\left(\varphi=15^{\circ}, d_{N}=12 \mathrm{~nm}\right.$ and $\left.d_{\mathrm{C} \alpha \beta}=3.5 \mathrm{~nm}\right)$ introduced in section A.1. Of note, $d_{\alpha 3}$ varies as $\theta$ changes because of a pulling constraint (see section B.1). With a fixed $\theta$ (i.e., $30^{\circ}$ ) the effect of molecular extension at zero force was investigated by varying extension from $0.5 \mathrm{~nm}$ to $3.5 \mathrm{~nm}$. These were selected by the average values of actual fitting results, which have been tested and confirmed as reasonable.

The free-energy landscape can be constructed by substituting the best-fit model parameters into the following equations (17): 


$$
\begin{gathered}
\Delta G^{*}(F)=\Delta G_{0}^{*}\left(1-\frac{2 \delta_{0}^{*} \gamma(F)}{3 \Delta G_{0}^{*}}\right)^{3 / 2}, \\
\delta^{*}(F)=\delta_{0}^{*}\left(1-\frac{2 \delta_{0}^{*} \gamma(F)}{3 \Delta G_{0}^{*}}\right)^{1 / 2},
\end{gathered}
$$

Thus, by using model parameters, the dissociation state coordinates relative to the bound state coordinates in the free-energy vs dissociation coordinate space can be defined as function of force. Note that this force-induced change of the barrier height should be under the condition for relatively small perturbation such that $\left|-\delta_{0}^{*} \gamma(F)\right|<\Delta G_{0}^{*}$. Since our fitting results showed that the average free-energy barrier height at zero force is $\sim 12 k_{\mathrm{B}} T\left(\left\langle\Delta G_{0}^{*}\right\rangle\right)$, the force range corresponding to a change of the barrier height $<10 k_{\mathrm{B}} T$ is reasonable for each data set, i.e., force range corresponding to energy barrier heights in the range of $2 k_{\mathrm{B}} T<\Delta G_{0}^{*}<23 k_{\mathrm{B}} T$.

To elucidate the biological relevance of the model parameters $\theta, n^{*}, d_{\mathrm{C} \alpha \beta}, \delta_{0}^{*}$, and $\Delta G_{0}^{*}$, we examine their changes with varying bond lifetime vs force data obtained from different TCR and pMHC interactions that induce a wide range of biological responses. Finding correspondence between a group of model parameters individually and/or collectively with the biological response would be considered to support the biological relevance of the model, because such correspondence suggest that the model can discriminate different TCR-pMHC interactions.

\section{A.5. Class I model constraints}

The purpose of this subsection is to check whether the constraints that model has are consistent within the model parameters obtained by fitting the model to data. Our experiments applied tensile force through the two ends of the TCR-pMHC complex, such that the force direction would always align with the line connecting the C-termini of the respective TCR and MHC molecules during dissociation, giving rise to the so-called pulling constraint $(2,3,5,9$, 26, 52). To formulate this pulling constraint in our model, we note that the pulling line is 
maintained so that the coordinate perpendicular to the force direction is invariant. As depicted in Fig. S4 $A$ using the horizontal line perpendicular to the pulling axis, several angle and length variables can be related using model parameters and several structural constants by:

$$
\left(d_{N}-d_{\mathrm{C} \alpha \beta}-d_{\alpha 3} \frac{\cos (\varphi+\theta)}{\cos \theta}\right) \sin \theta=d_{\alpha 3}\left(\frac{\sin \varphi}{\cos \theta}+\cos \left(2 \psi+\varphi+\theta-\frac{\pi}{2}\right)\right),
$$

where $\psi$ is the angle in an isosceles triangle constructed by rotating the $\alpha 3$ domain. By assuming that the $\alpha 3$ domain would be aligned with the force, we estimate that the angle in the last term is near $90^{\circ}$, i.e., $2 \psi+\varphi+\theta-\frac{\pi}{2} \approx \frac{\pi}{2}-\delta$ where $\delta \ll \frac{\pi}{2}$. Under this small angle assumption, Eq. S9a can be approximated by:

$$
d_{\alpha 3} \approx \frac{d_{N}-d_{\mathrm{C} \alpha \beta}}{\cot \theta \sin \varphi+\cos \varphi+\sin \delta / \sin \theta} .
$$

Upon inversing Eq. S9b, we found that the tilting angle $\theta$ is function of the end-to-end distance of the $\alpha 3$ domain, i.e., $\theta=f\left(d_{\alpha 3}\right)$. Setting $\delta=25^{\circ}$, which seems reasonable as it approximates the maximum value of $\varphi$, the structural parameters obtained by fitting are scattered in-between two black curves on the $d_{\alpha 3}-\theta$ plane marked as pulling constraint: $\left[d_{N}=12.5 \mathrm{~nm}, d_{\mathrm{C} \alpha \beta}=3.62 \mathrm{~nm}, \varphi=0^{\circ}\right]$ and $\left.\left[d_{N}=11.5 \mathrm{~nm}, d_{\mathrm{C} \alpha \beta}=3.42 \mathrm{~nm}, \varphi=25^{\circ}\right]\right)$ (Fig. 3F).

Another important constraint is the tilting constraint resulted from the asymmetric unfolding and stretching of the interdomain links between the TCR constant and variable domains. This constraint is introduced in the model to account for the FG-loop's potential regulatory effect on catch bond. Notwithstanding the total number of unfolded amino acids $n^{*}$ can be determined by the validation procedure demonstrated in A.2, its breakdown into the number of unfolded amino acids for MHC $\left(n_{\mathrm{p}, \mathrm{MHC}}\right)$ and TCR $\left(n_{\mathrm{p}, \mathrm{TCR}}\right)$ remains undetermined. To do this, known structures from $P D B$ were used to determine $n_{\mathrm{p}, \mathrm{MHC}}$ first. Briefly, by matching $d_{\alpha 3}$ (Fig. S4B and $C$ ) with the end-to-end distance between C-terminal end of $\alpha 3$ 
domain and certain point following known $P D B$ structure, the exact starting position of partial unfolding in $\mathrm{MHC}$ can be found following additional assumption that unfolding starts from $\mathrm{C}$ terminal end of $\alpha 2$ domain towards $\alpha 3$ domain (Fig. S4B). Thus, $n_{\mathrm{p}, \mathrm{TCR}}$ can be simply calculated as $n_{\mathrm{p}, \mathrm{TCR}}=n^{*}-n_{\mathrm{p}, \mathrm{MHC}}$. Upon combining all information, the tilting angle $\left(\theta_{\mathrm{TCR}}\right)$ of variable domains of TCR can be described by simple trigonometrical function:

$$
\theta_{\mathrm{TCR}} \approx \tan ^{-1}\left(\frac{n_{\mathrm{p}, \mathrm{TCR}} l_{\mathrm{c}}}{W_{\mathrm{TCR}}}\right)
$$

where $W_{\mathrm{TCR}}$ is width of two interdomain hinges of the TCR $\alpha$ - and $\beta$-subunits measured from the crystal structures (Fig. S4A, tilting constraint). In this work we use $W_{\mathrm{TCR}}=3.7 \pm 0.3 \mathrm{~nm}$ as a representative width due to structure-to-structure variation. Thus, by comparing the tilting angle of the bonding interface $\theta$ (model parameter) to the tilting angle of the TCR $\theta_{\mathrm{TCR}}$ (derived from another model parameter $n_{\mathrm{p}, \mathrm{TCR}}$ and structural constants $W_{\mathrm{TCR}}$ and $l_{\mathrm{c}}$ ), we can check the validity of tilting constraint using linear regression in $\left(\theta_{\mathrm{TCR}}, \theta\right)$ domain (Fig. $\left.\mathrm{S} 4 D\right)$.

\section{B. Kinetic model for TCR-pMHC-II}

\section{B.1. Development, validation, and characterization}

The purpose of this subsection is to present details of the development, validation, and characterization of the TCR-pMHC-II model omitted in the main body for simplicity, in a similar fashion as the TCR-pMHC-I model described in Section A. The two models share exact the same framework but have different detailed form of the characteristic extension change $\delta_{\mathrm{z}}(f)$ (Eq. S5). Comparing to the TCR-pMHC-I complex, the TCR-pMHC-II complex has different docking domains and pulling geometries (one vs two transmembrane domains on both TCR and MHC). For this reason, we assumed that the force-induced bonding interface tilting angle $(\theta)$ would be much smaller in the TCR-pMHC-II than TCR-pMHC-I complex. The extension at the bound state can be defined as the end-to-end distance between both end-points 
identified by crystal structures (E8: 2IAM, 2IAN and 2B4 (as the substitution of 3.L2): 6BGA, 3QIB):

$$
z(f ; 0)=d_{\mathrm{N}}(f)+d_{\text {linker }}(f),
$$

where $d_{\mathrm{N}}$ is set to be $12.3 \mathrm{~nm}$ based on the crystal structures and $d_{\text {linker }} \sim 9.4 \mathrm{~nm}$ represents the linker (e.g., a leucine zipper) engineered at the C-termini of soluble pMHC-II constructs to stabilize both the MHC $\alpha$ - and $\beta$-subunits, which is often used in experiments for measuring TCR-pMHC-II catch bonds. To account for domain rotation resulted from partial unfolding inside the TCR-pMHC-II complex, we introduce one more variable as the tilting angle of the TCR constant domains $(\varphi)$. Using the tilting constraint similarly to that in the class I model, this angle can be approximately described by structural parameters $\left(d_{\text {B.I }}, \theta\right)$ and model constants (see subsection B.2). In short, each component in the right-hand side of Eq. S11 can be expressed by the model parameters $\left(d_{\mathrm{B} . \mathrm{I}}, \theta, n^{*}\right)$, and model constants as follows.

$$
\begin{gathered}
z_{\mathrm{B} . \mathrm{I}}(f)=\left(d_{\mathrm{B} . \mathrm{I}}(f)+z_{\mathrm{p}, \mathrm{MHC}}(f)\right) \cos \theta, \\
z_{\mathrm{C} \alpha \beta}(f)=\left(d_{\mathrm{C} \alpha \beta}(f)+z_{\mathrm{p}, \mathrm{TCR}}(f)\right) \cos \varphi, \\
z_{\text {linker }}(f)=d_{\text {linker }}(f) \cos \theta,
\end{gathered}
$$

where $z_{\mathrm{p}, \mathrm{MHC}}(f)$ and $z_{\mathrm{p}, \mathrm{TCR}}(f)$ are respective extensions of unfolded polypeptides given by $n_{\mathrm{p}, \mathrm{MHC}} l_{\mathrm{c}} z_{u, p}(f)$ and $n_{\mathrm{p}, \mathrm{TCR}} l_{\mathrm{c}} z_{u, p}(f)$, respectively, $d_{\mathrm{B} . \mathrm{I}}$ is the length of structure consisting of the MHC and the TCR variable domains, a nd other parameters defined previously. Finally, the rate coefficient of the TCR-pMHC-II dissociation can be developed by employing the same framework (see Eq. S6). However, when applying the model to experimental data, we can use the constraints of the TCR-pMHC-II interaction to make the model much simpler (see section B.2).

Validation of the class II model follows exactly the same procedure as that used in the validation of the pMHC I model, which is done by checking self-consistent through the 
definition of the reaction coordinate. By varying the $n_{\mathrm{p}, \mathrm{MHC}}$ from 0 to 10 (see details explained in section B.2), the molecular extension at zero force $\left(\delta_{0}^{*}\right)$ can be estimated by using the contour length-change $\left(\delta_{1}\right)$ along the dissociation coordinate:

$$
\delta_{\mathrm{l}}=n_{\mathrm{p}, \mathrm{MHC}} l_{\mathrm{c}}+\Delta \rightarrow \delta_{0}^{*}
$$

where $\Delta\left(d_{\mathrm{B} . \mathrm{I}}, \theta\right)<0.2 \mathrm{~nm}$ because $\theta<10^{\circ}$. The best-fit model parameters can be determined by finding a subset of best-fit parameters among possible $n_{\mathrm{p}, \mathrm{MHC}}$ values that match the contour length-change along the dissociation coordinate $\left(\delta_{1}\right)$ to the width of the free-energy well at zero force $\left(\delta_{0}^{*}\right)$, i.e., finding $n_{\mathrm{p}, \mathrm{MHC}}$ such that $\delta_{\mathrm{l}}\left(n_{\mathrm{p}, \mathrm{MHC}}\right) \rightarrow \delta_{0}^{*}$ (see Table S4).

To characterize the class II model, we examine the model predictions by varying the model parameters one by one while fixing the others as constants. For example, to investigate force-induced bonding interface titling, we fixed the other model parameters $\left(\right.$ i.e., $n^{*}=4, \delta_{0}^{*}=$ $1.2 \mathrm{~nm}, k_{0}=10 \mathrm{~s}^{-1}$, and $\left.\Delta G_{0}^{*}=11.5 k_{\mathrm{B}} T\right)$ and structural constants $\left(d_{\mathrm{N}}=12 \mathrm{~nm}, d_{\mathrm{B} . \mathrm{I}}=12.3\right.$ $\mathrm{nm}$, and $d_{\text {linker }}=9.4 \mathrm{~nm}$ for pMHC-II constructs that have linkers). As another example, we fixed $\theta=0^{\circ}$ or $3^{\circ}$ and examined the effect of the molecular extension at zero force by varying $\delta_{0}^{*}$ from $0.3-1.8 \mathrm{~nm}$. The constants used for model characterization were selected by their averages from the corresponding values used to fit actual experiments. The parameters for freeenergy landscape construction, the energy barrier height $\left(\Delta G^{*}\right)$ and energy well width $\left(\delta^{*}\right)$ as functions of force, are given by Eq. S8, exactly the same as the pMHC I model.

\section{B.2. Class II model constraints}

The purpose of this subsection is to describe the constraints of the class II model, which share similar ideas to those of the class I model (e.g., pulling and tilting constraints) but differ in their specific expressions. To formulate the pulling constraint, we again used the fact that the pulling force direction must aligns with the line connecting to the C-termini of the TCR 
and pMHC molecules so that the coordinate perpendicular to the force direction is invariant. Using model parameters and structural constants, this pulling constraint can be written as follows:

$$
\left(d_{\mathrm{B} . \mathrm{I}}+z_{\mathrm{p}, \mathrm{MHC}}+d_{\text {linker }}\right) \sin \theta=\left(d_{\mathrm{C} \alpha \beta}+z_{\mathrm{p}, \mathrm{TCR}}\right) \sin \varphi
$$

which can be solved for $z_{\mathrm{p}, \mathrm{TCR}}$ explicitly:

$$
z_{\mathrm{p}, \mathrm{TCR}}=\left(d_{\mathrm{B} . \mathrm{I}}+z_{\mathrm{p}, \mathrm{MHC}}+d_{\text {linker }}\right) \frac{\sin \theta}{\sin \varphi}-d_{\mathrm{C} \alpha \beta} .
$$

By combining Eq. S12 and S14, the total extension $(z)$ can be calculated as the sum of all component extensions $\left(\sum_{i} z_{i}\right)$ at dissociation $\left(\delta_{l}>0\right)$ :

$$
z\left(f ; \delta_{l}\right)=\left(d_{\mathrm{B} . \mathrm{I}}(f)+z_{\mathrm{p}, \mathrm{MHC}}(f)+d_{\text {linker }}(f)\right)(\cos \theta+\sin \theta \cot \varphi) .
$$

This equation states that only does the number of unfolded amino acids in MHC ( $\left.n_{\mathrm{p}, \mathrm{MHC}}\right)$ affect extension change during transition. The total number of unfolded amino acids $\left(n^{*}\right)$ can be estimated by Eq. S14b:

$$
n^{*} \approx\left|\frac{\left(d_{\mathrm{B} . \mathrm{I}}+d_{\text {linker }}\right) \frac{\sin \theta}{\sin \varphi}-d_{\mathrm{C} \alpha \beta}}{l_{\mathrm{c}}}+\left(1+\frac{\sin \theta}{\sin \varphi}\right) n_{\mathrm{p}, \mathrm{MHC}}\right| .
$$

Total number of unfolded amino acids from both TCR and MHC, $n^{*}$, can be approximately calculated using contour lengths of length components at force-free state.

By assuming small angle perturbation, which seems reasonable, we further reduce the number of model parameters after relating the tilting angle of the TCR constant domain $(\varphi)$ and the titling angle of the bonding interface by the following equation:

$$
\cot \varphi \approx \frac{d_{N}-d_{\mathrm{B} . \mathrm{I}}}{d_{\mathrm{B} . \mathrm{I}}+d_{\text {linker }}} \cot \theta
$$

Thus, all terms including $\varphi$ can be re-expressed by using Eq. S17.

Additionally, the tilting constraint can be expressed as follows: 


$$
\frac{\tan ^{-1}\left(\frac{n_{\mathrm{p}, \mathrm{TCR}} l_{\mathrm{c}}}{W_{\mathrm{TCR}}}\right)}{\theta+\varphi} \approx 1 .
$$

Thus, by using Eq. S15 and S17, only 4 fitting parameters, two structural parameters $\left(d_{\text {B.I }}, \theta\right)$ and two biophysical parameters $\left(k_{0}, \delta_{0}^{*}\right)$, were used to fit the class II model to data.

\section{A general biophysical limit of model parameters}

The purpose of this section is to describe a general biophysical limit that constrain the fitted model parameters, which is used in Fig. 5 to accept the correct model application to data of matched MHC class and reject incorrect model application to data of matched MHC class. The idea is that, even if the model is capable of fitting experimental data and the parameters are self-consistency with one another within the model, their values should be within wellknown limits. A prototypical example of such a biophysical limit involves the molecular extension per unfolded amino acids. It follows from Eqs. S7 and S13 that the average molecular extension at zero force over all data $\left(\left\langle\delta_{0}\right\rangle\right)$ should be a linear function of $n^{*}$ such that $\left\langle\delta_{0}\right\rangle=$ $a n^{*}+b$ where $a$ is the slope and $b$ is the $y$-axis intercept. $b$ can be determined from the slip bond data because slip bonds are not expected to have unfolded amino acids but still have a nonzero extension. Given that the contour length of a single amino acid has a small range $(\sim 0.4$ $\pm 0.02 \mathrm{~nm} / \mathrm{a} . \mathrm{a})(17,29,30)$, the average molecular extension $\left(\left\langle\delta_{0}\right\rangle\right)$ per unfolded amino acids $\left(n^{*}\right)$ should be bounded by:

$$
0<a<0.4
$$

Imposing this range limit of $\left\langle\delta_{0}\right\rangle / a$. $a$. would enables us to rule out inappropriate application of the model even if such application could achieve reasonable level of goodnessof-fit. An exceedingly large value of $a(>0.4 \pm 0.02 \mathrm{~nm} / \mathrm{a}$.a) estimated by the model would mean that the model is inappropriate for the data. 
Conversely, a nearly zero estimate of $a$ would indicate that the model is inappropriate for catch-slip bond data because, for the model to fit catch-slip bonds, it requires $n^{*}>0$ (see Figs. $1 C-G$ and $2 D$ ). A parameter estimation of $a \approx 0$ indicates the lack of dependence of the model behavior on $n^{*}$, which abolishes the model's ability to predict TCR signaling and antigen discrimination, making the model irrelevant to biology.

The sturdier class II than class I pMHC structure also precludes large rotation during conformational change at transition state, leaving only unfolding and stretching along the force. Thus, the average molecular extension per amino acid should be close to $0.4 \mathrm{~nm} / \mathrm{a}$.a. On the other hand, $a \approx 0$ is expected from fitting the slip-only data because such data correspond to the $n^{*}=0$ case, which makes it difficult to robustly estimate the correct $a$ value. Thus, the average molecular extension per amino acid should be well-correlated with each other (high level of goodness-of-fit as measured by $R^{2}$ ) and in the range between 0 to 0.4 nm/a.a. In Fig. 5, we used these criteria to test the appropriateness of cross-applying the class I model to class II data and vice versa, showing that it is appropriate to use either model to fit matched data but inappropriate to use either model to fit mismatch data. 


\section{Supplemental Figures}

A
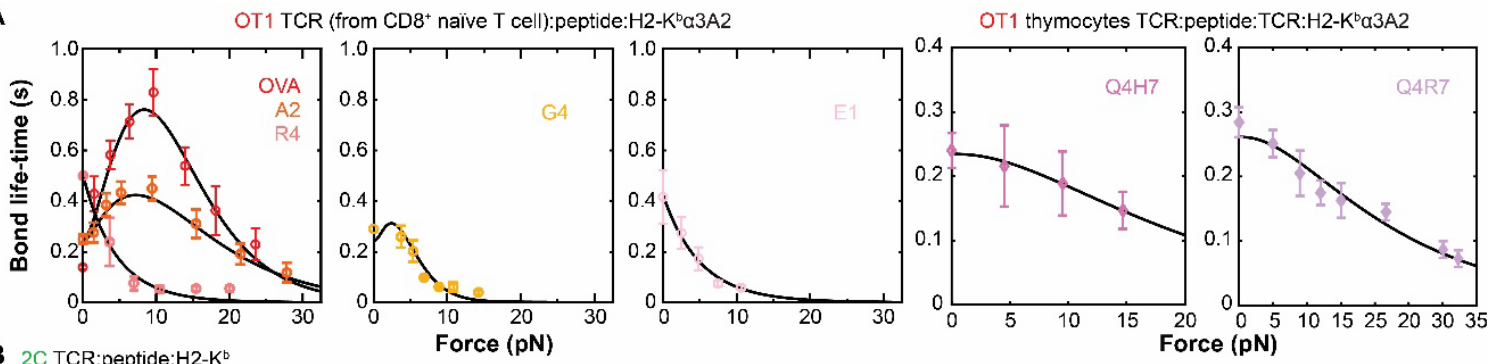

B 2C TCR:peptide:H2-K
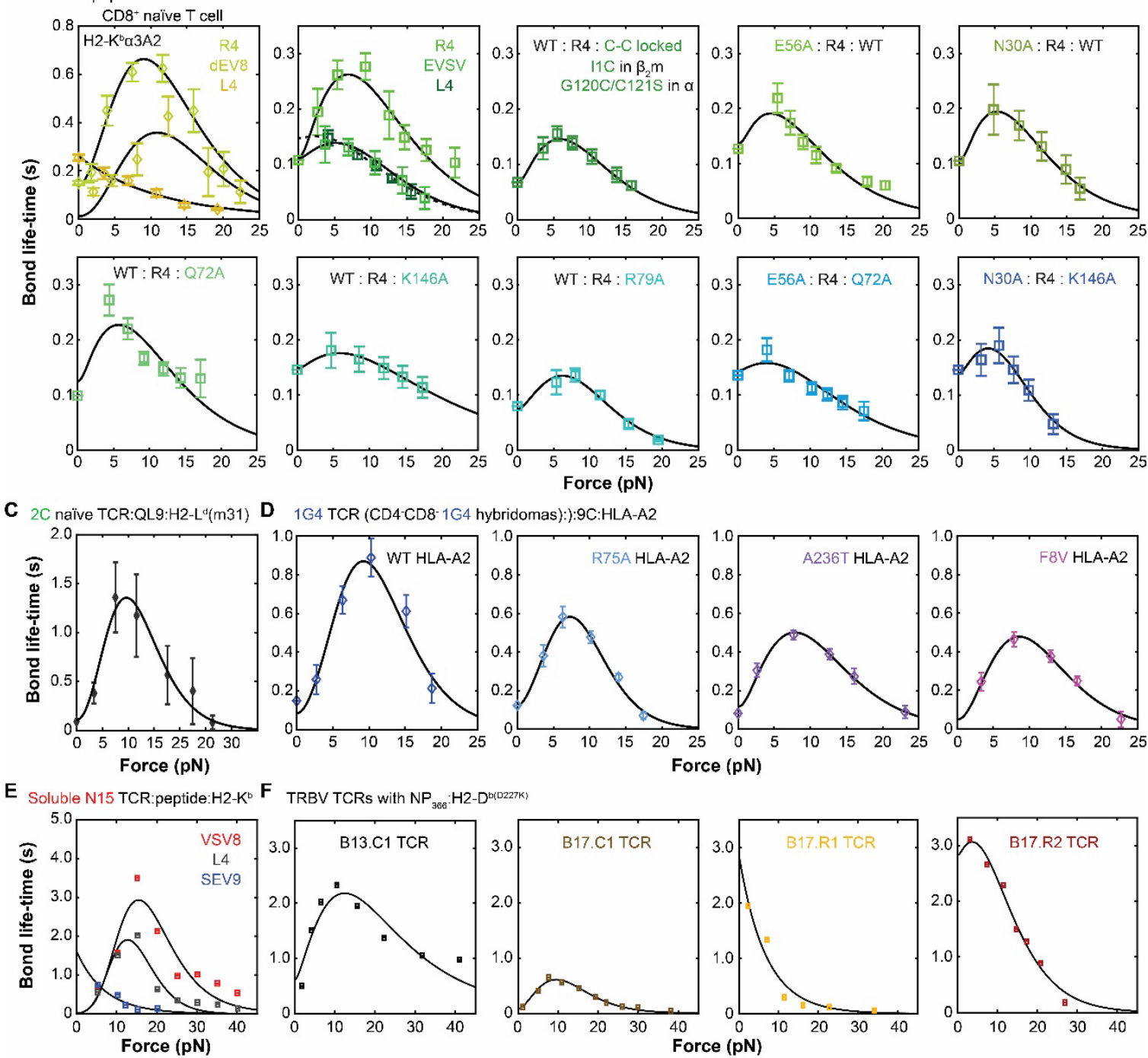

Fig. S1. Fitting class I model to data of TCR-pMHC-I bond lifetime vs force. Fitting of theoretical $1 / k(F)$ curves to experimental bond lifetime vs force data (points, mean \pm sem) of 3 TCRs and their mutants interacting with different pMHCs as described below (re-analyzed from $(2,5,9)$ ). (A) OT1 TCR expressed on $\mathrm{CD} 8^{+}$naïve $\mathrm{T}$ cells (first three panels) or $\mathrm{CD} 4^{+} \mathrm{CD} 8^{+}$ thymocytes (last two panels) interacting with indicated $\mathrm{p}: \mathrm{H} 2-\mathrm{K}^{\mathrm{b}} \alpha 3 \mathrm{~A} 2$. (B) $2 \mathrm{C}$ TCR expressed on $\mathrm{CD}^{+}$naïve $\mathrm{T}$ cells interacting with indicated $\mathrm{p}: \mathrm{H} 2-\mathrm{K}^{\mathrm{b}} \alpha 3 \mathrm{~A} 2$ (top, $1^{\text {st }}$ panel) or on $\mathrm{CD} 8^{-}$ 
hybridomas interacting with indicated peptides presented by WT (top, $2^{\text {nd }}$ panel) or disulfatelocked mutant (top, $3^{\text {rd }}$ panel) $\mathrm{H} 2-\mathrm{K}^{\mathrm{b}}, 2 \mathrm{C}$ TCR with indicated point mutations expressed on CD8 ${ }^{-}$hybridomas interacting with $\mathrm{R} 4: \mathrm{H} 2-\mathrm{K}^{\mathrm{b}}$ (top, $4^{\text {th }}$ and $5^{\text {th }}$ panels), 2C TCR expressed on CD8- hybridomas interacting with $\mathrm{R} 4$ peptide presented by $\mathrm{H} 2-\mathrm{K}^{\mathrm{b}}$ with indicated point mutations (bottom, $1^{\text {st }}-3^{\text {rd }}$ panels), or $2 \mathrm{C}$ TCR with indicated point mutations expressed on CD8- hybridomas interacting with $\mathrm{R} 4$ peptide presented by $\mathrm{H} 2-\mathrm{K}^{\mathrm{b}}$ with indicated point mutations (bottom, $4^{\text {th }}$ and $5^{\text {th }}$ panels). (C) $2 \mathrm{C}$ TCR expressed on $\mathrm{CD} 8^{+}$naïve $\mathrm{T}$ cells interacting with indicated p:H2- $\mathrm{L}^{\mathrm{d}}(\mathrm{m} 31)$ with truncated $\alpha 3$ domain. (D) 1G4 TCR expressed on CD8hybridomas interacting with indicated p:HLA-A2. The first panel of A and the first two top panels of B are replotted from Fig. $2 A$ and $B$ for completeness. (E) Purified N15 TCR $\alpha \beta$ interacting with indicated p:H2-K $\mathrm{K}^{\mathrm{b}}$. (F) TRBV TCRs of canonical (B13.C1 and B17.C1) and reverse (B17.R1 and B17.R2) docking orientation on pMHC-I expressed on hybridomas interacting with $\mathrm{NP}_{366}: \mathrm{H} 2-\mathrm{D}^{\mathrm{bD} 227 \mathrm{~K}}$. See Table S3 for a list of the interacting molecules. 

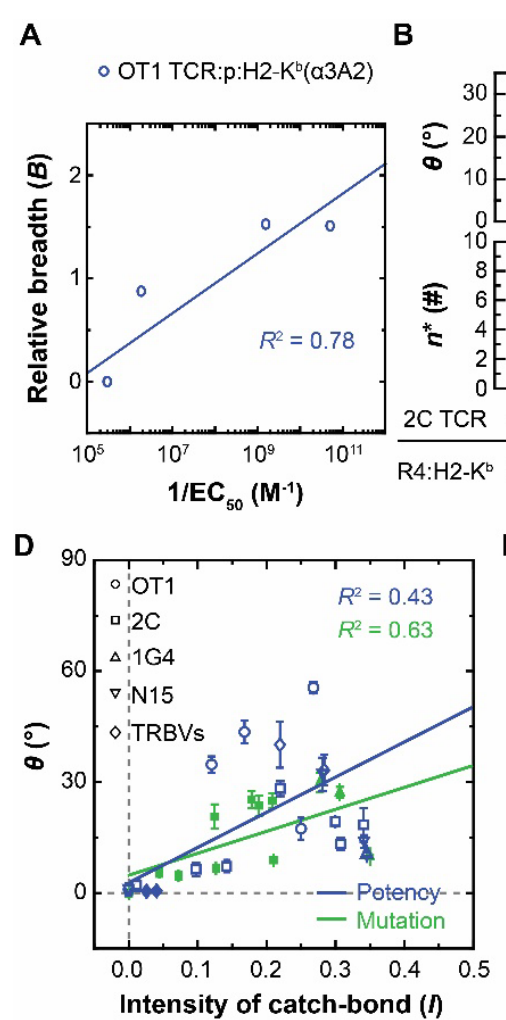

B

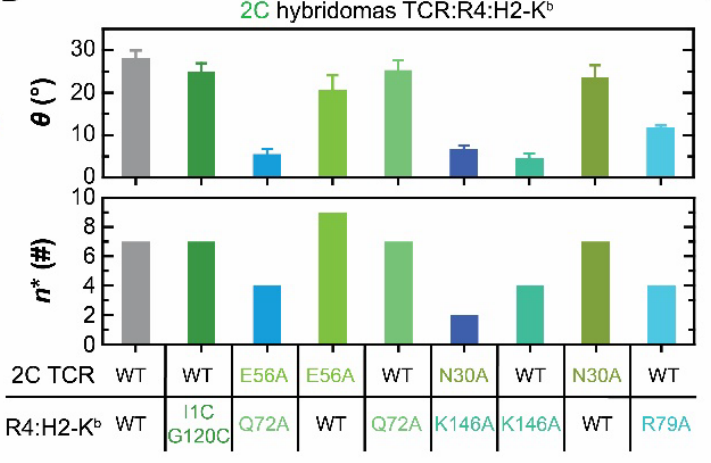

C

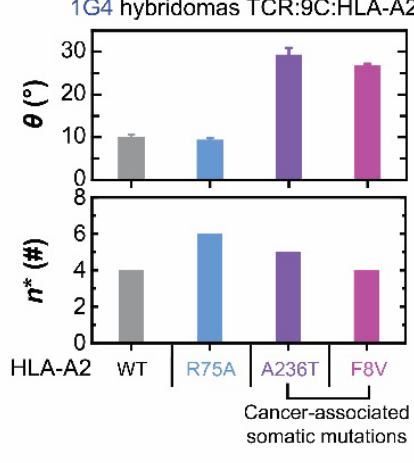

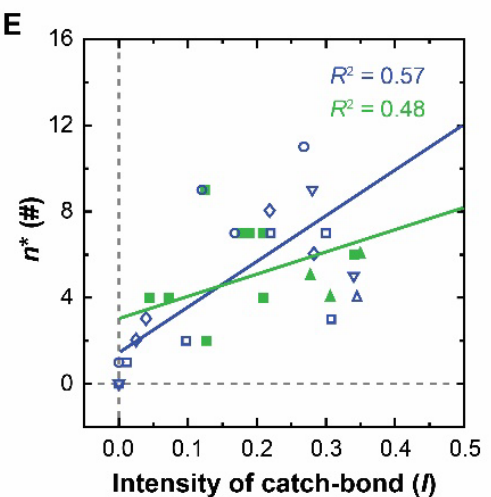

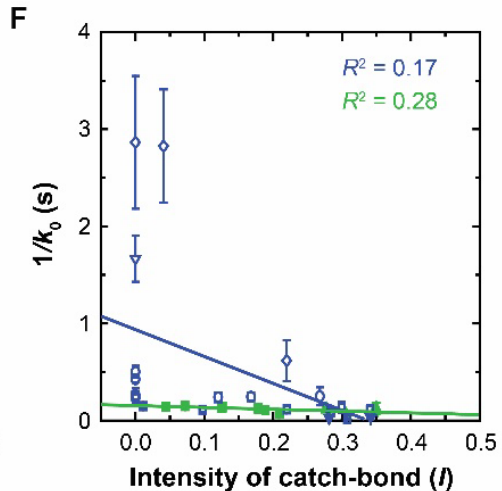

Fig. S2. Correlation of model parameters with pMHC-I pMHC-I biological activity and

TCR bond type. (A) Relative breath vs the peptide dose required to stimulate half maximal of OT1 T cell proliferation. (B, C) Model parameters $\boldsymbol{\theta}$ (the tilted angle of the bonding interface, upper) and $\boldsymbol{n}^{*}$ (the number of unfolded amino acids, lower) that best-fit the data in Fig. S1B and $D$ are plotted vs the indicated WT and mutant 2C (B) and 1G4 (C) TCRs with their indicated pMHCs. (D-F) Scattergrams of $\boldsymbol{\theta}(\mathrm{E}), \boldsymbol{n}^{*}(\mathrm{~F})$, and $\mathbf{1} / \boldsymbol{k}_{\mathbf{0}}(\mathrm{G})$ vs $\boldsymbol{I}=\boldsymbol{L} /(\mathbf{1}+\boldsymbol{B})$ (catch bond intensity) are plotted using the data from Figs. $2 E \& 2 F, 3 B$, and $\mathrm{S} 2 B \& \mathrm{~S} 2 C$ to examine correlation. Blue-open symbols indicate data of known $\mathrm{T}$ cell biological activities (ligand potencies) induced by the corresponding TCR-pMHC-I interactions that correlate to catch bond intensity. Green-closed symbols indicate data of known effects on catch bond metrics by targeted mutations on the TCR, MHC, or both. See Table S3 for a list of the interacting molecules. 

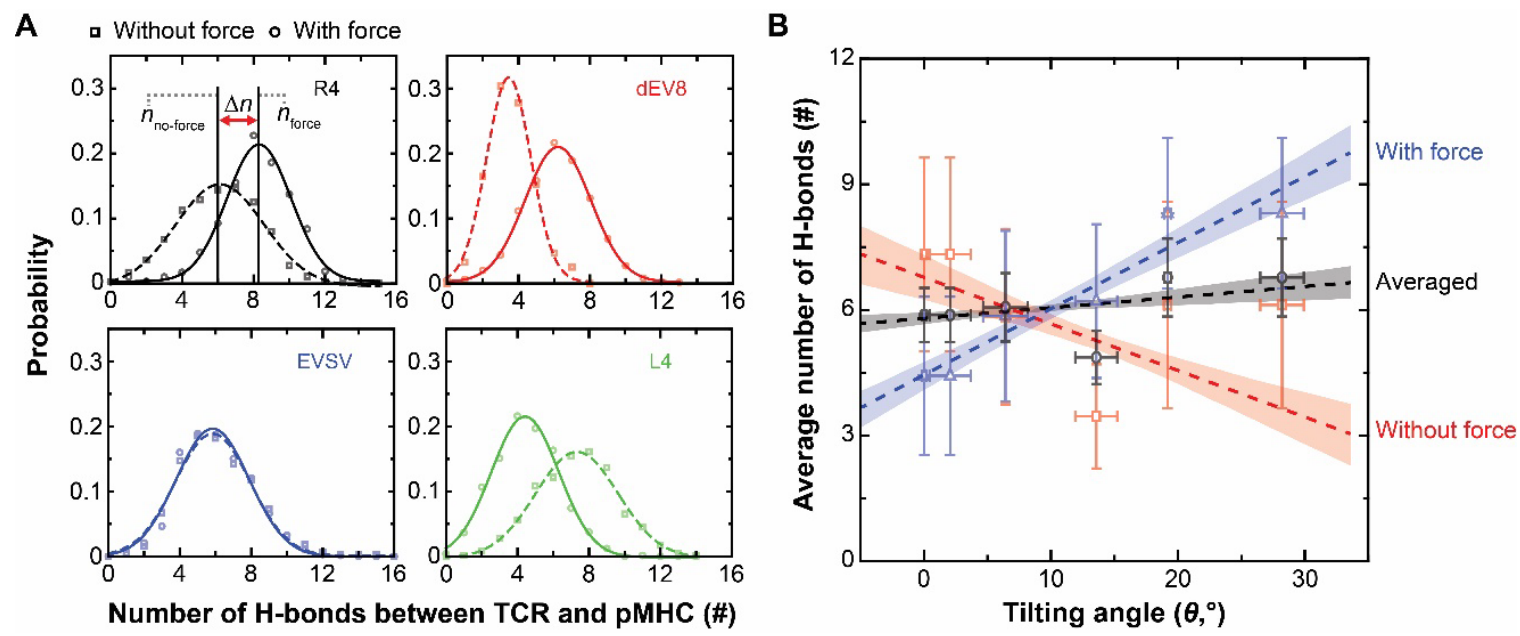

Fig. S3. Hydrogen (H) bond distributions at the TCR-pMHC-I bonding interface. (A) Hbond distributions at bonding interface between 2C TCR and the pMHCs indicated at each panel (R4, dEV8, EVSV, or L4) in the presence (solid curves) or absence (dotted curves) of the force (obtained by re-analysis of SMD simulation results from (5) and fitted by Gaussian functions). (B) Plots of average number of $\mathrm{H}$-bonds with respect to tilting angle of bonding interface in the absence (red) and presence (blue) of force as well as their average (gray). Average numbers of $\mathrm{H}$-bonds were determined as mean value for each Gaussian distribution at each titling angle. Dotted-lines are linear fits and shaded regions are 95\% confidential intervals. 
A

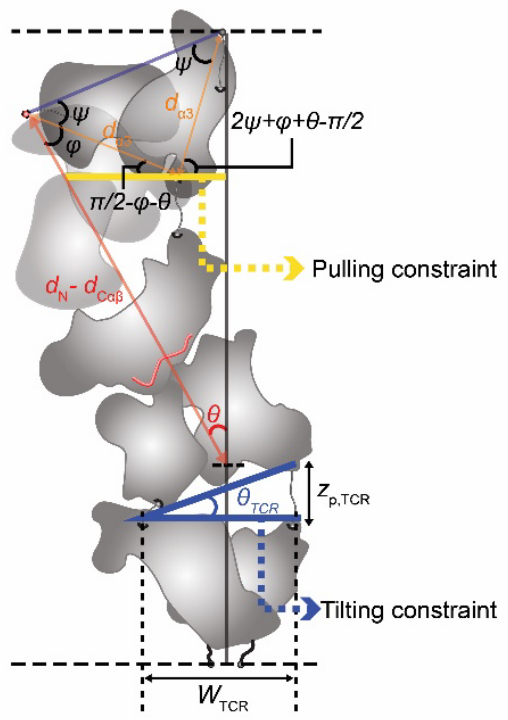

B

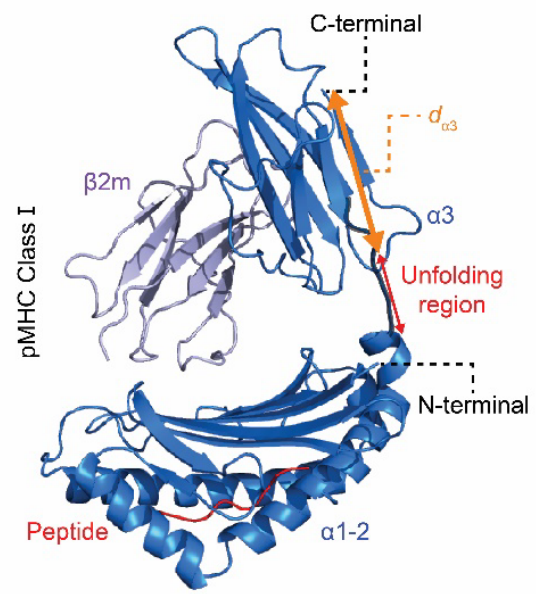

C

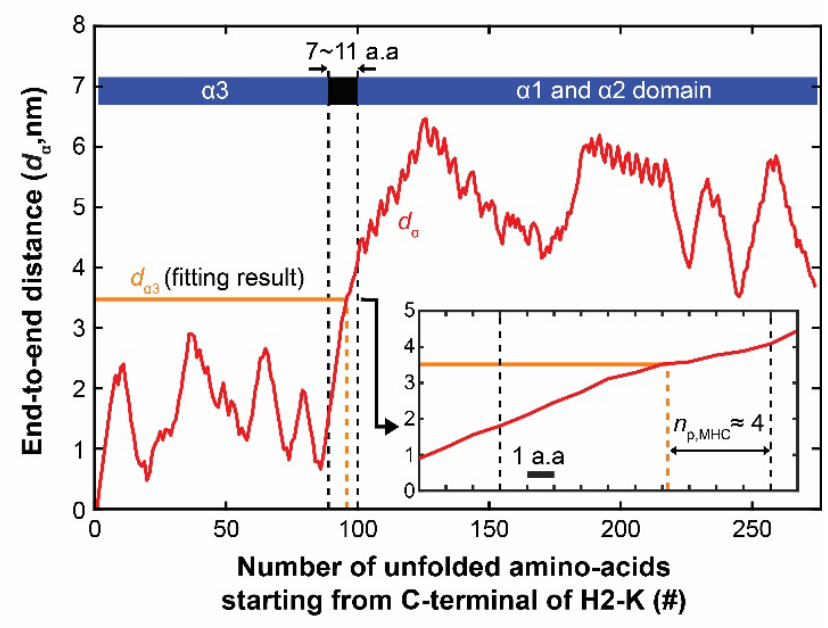

D

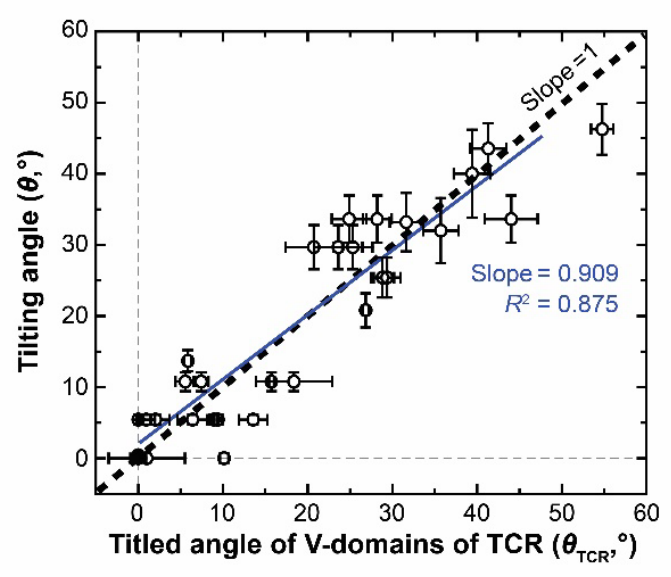

Fig. S4. Pulling and tilting constraints of class I model. (A) An illustration of both pulling and tilting constraints. Length of yellow line was used to set up the equation of the pulling constraint between $\boldsymbol{d}_{\boldsymbol{\alpha} \mathbf{3}}$ and $\boldsymbol{\theta}$. The tilted angle from the blue lines was used to check asymmetric stretching of TCR. (B) A pMHC-I structure based on PDB code 2CKB. Partial unfolding of $\mathrm{MHC}$ is assumed to start from the end of the $\alpha 1-\alpha 2$ domain towards the $\alpha 3$ domain as suggested by SMD simulation (5). (C) Representative end-to-end distance of $\boldsymbol{d}_{\boldsymbol{\alpha} 3}$ vs the number of unfolded amino acids. The distance is calculated from C-terminal end of the $\boldsymbol{\alpha} 3$ domain based on the structure shown in $\mathrm{B}$. Distance is calculated using $P B D$ structure for each construct. $\boldsymbol{d}_{\boldsymbol{\alpha} 3}$ distance obtained from fitting the model to data is converted to $\boldsymbol{n}_{\mathbf{p}, \text { MHC }}$ (inset). (D) Tilted angle of the TCR variable domains $\left(\boldsymbol{\theta}_{\mathbf{T C R}}\right)$ vs the titled angle of bonding interface $(\boldsymbol{\theta})$ as one of the fitting parameters. The validity of asymmetric stretching of TCR was checked by the linear relationship between the two angles. 
bioRxiv preprint doi: https://doi.org/10.1101/2022.01.17.476694; this version posted January 18, 2022. The copyright holder for this preprint (which was not certified by peer review) is the author/funder. All rights reserved. No reuse allowed without permission. 
A

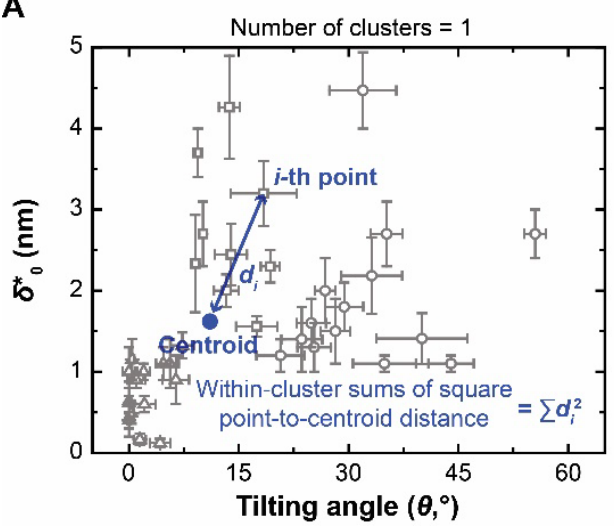

B

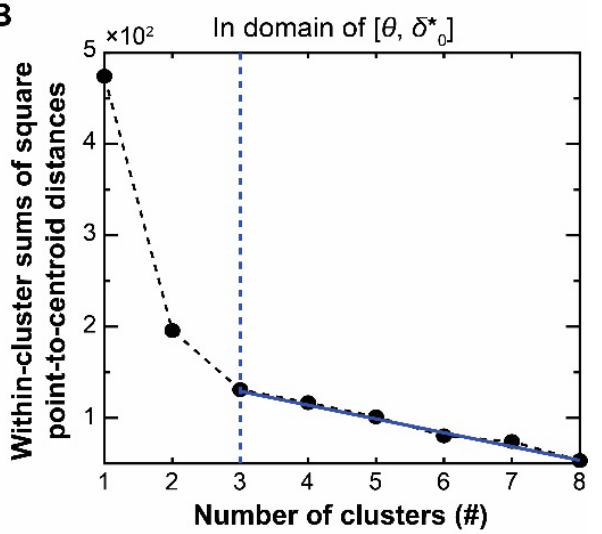

Fig. S5. Determining the number of clusters in parameter space. (A) An illustration of definition of point-to-centroid distance (Euclidean distance) when the number of clusters is 1 . (B) Sum of all squared Euclidean distances vs number of possible clusters. Point of abrupt reduction of steepness of slope leading to a plateau (blue solid-line) was identified a number of clusters (blue dotted-line). 

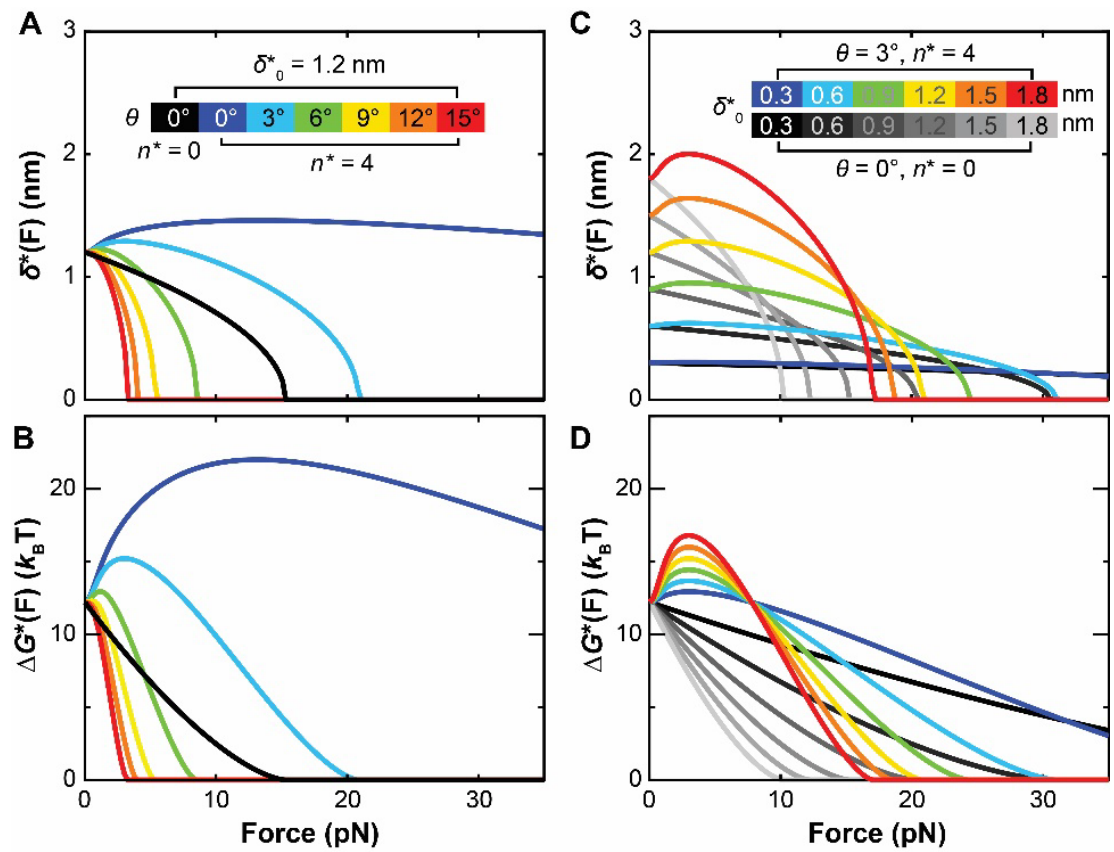

Fig. S6. Characterization of the energy landscape of TCR-pMHC-II dissociation. (A-D)

Plots of transition state location $\boldsymbol{\delta}^{*}(\mathrm{~A}, \mathrm{C})$ and height of energy barrier $\boldsymbol{\Delta} \boldsymbol{G}^{*}(\mathrm{~B}, \mathrm{D})$ vs force $\boldsymbol{F}$ for changing $\boldsymbol{\theta}$ and $\boldsymbol{n}^{*}(\mathrm{~A}, \mathrm{~B})$ or $\boldsymbol{\delta}_{\mathbf{0}}^{*}$ and $\boldsymbol{n}^{*}(\mathrm{C}, \mathrm{D})$ while keeping $\boldsymbol{\delta}_{\mathbf{0}}^{*}=\mathbf{1 . 2} \mathrm{nm}(\mathrm{A}, \mathrm{B})$ or $\boldsymbol{\theta}=$ $\mathbf{0}^{\circ}, \mathbf{3}^{\circ}(\mathrm{C}, \mathrm{D})$. Inset color bars indicate parameter values used to plot the color-match theoretical curves. 
$\mathbf{A}$

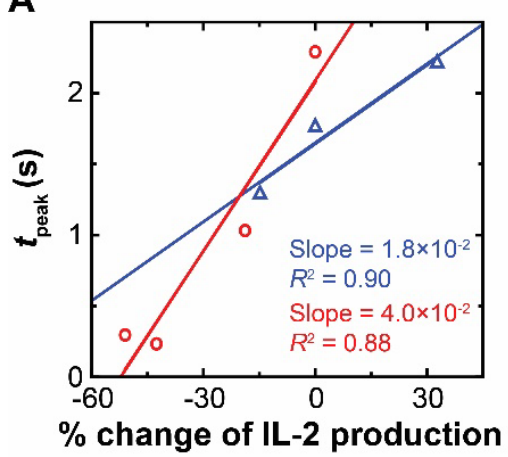

B

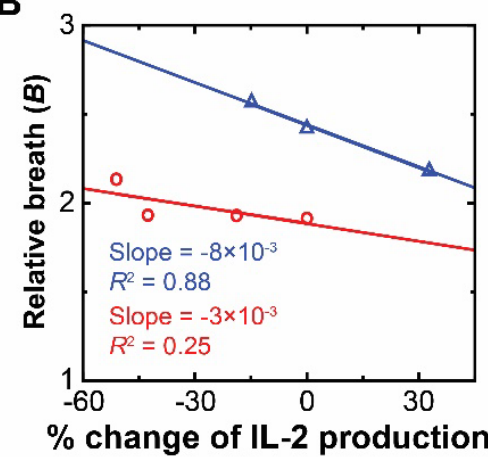

Fig. S7. Correlation of metrics of TCR-pMHC-II bond lifetime vs. force curves with T cell biological activity. Dimensional metric, $\boldsymbol{t}_{\text {peak }}$ (A) and relative breath of catch-slip bond $B$ (B) vs \% change (relative to WT) in the peptide dose required to achieve half-maximum hybridoma IL-2 production $\left(1 / \mathrm{EC}_{50}\right)(27)(3 . \mathrm{L} 2, \mathrm{red})$ or in the area under the dose response curve (AUC) (28) (2B4, blue) plots. 
bioRxiv preprint doi: https://doi org/10.1101/2022.01.17.476694; this version posted January 18, 2022. The copyright holder for this

Fitting curves by TCR:pMHC classI model

A OT1 naïve TCR:peptide:H2-K ${ }^{b} \alpha 3$ A2

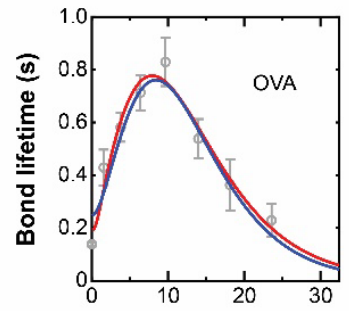

C $2 \mathrm{C} \mathrm{TCR}$ (from $2 \mathrm{C}$ hybridomas):peptide: $\mathrm{H}_{2}-\mathrm{K}^{\mathrm{b}}$
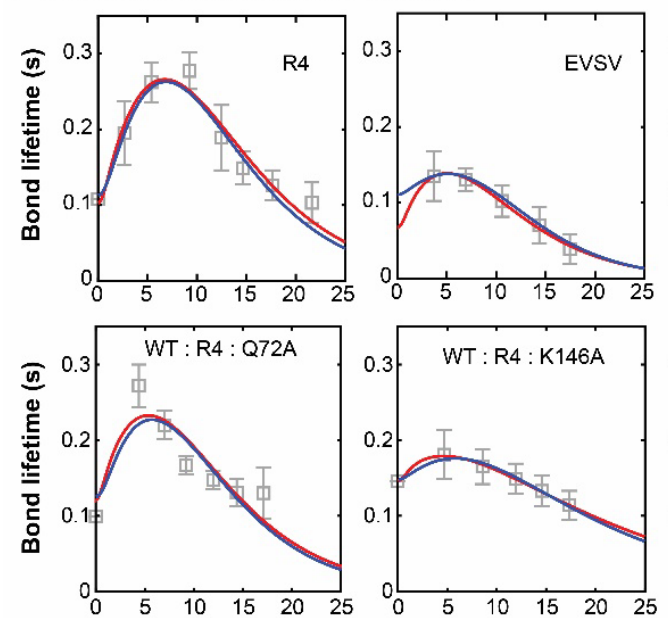

D $1 \mathrm{G} 4 \mathrm{TCR}$ (from hybridomas):9C:HLA-A2
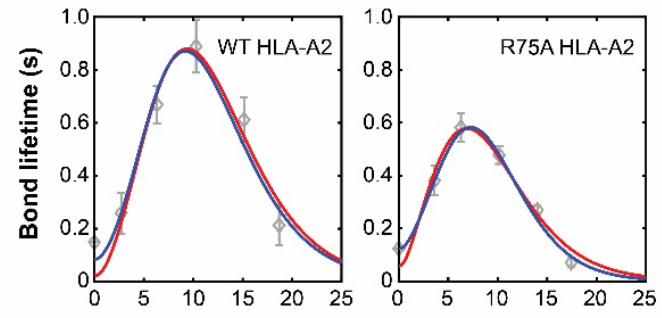

$\mathbf{F}$

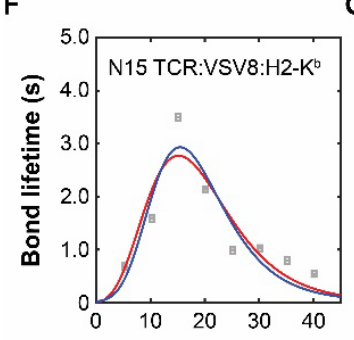

G 3.L2 TCR:peptide:IE ${ }^{K}$

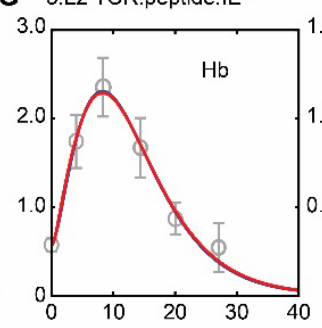

H 2B4 TCR:K5:IEK
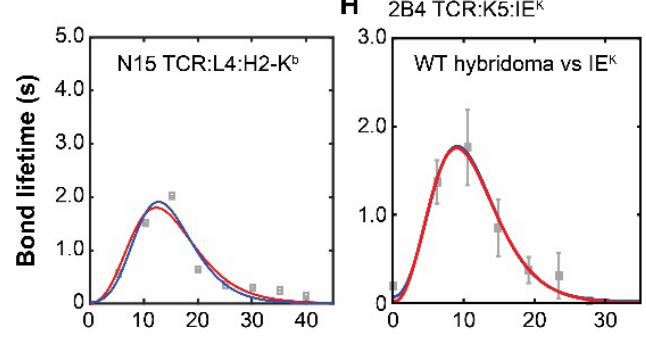

Fitting curves by TCR:pMHC classII model

B $2 \mathrm{C} \mathrm{TCR}$ (from naïve $\mathrm{CD} 8^{+} \mathrm{T}$ cell):peptide: $\mathrm{H} 2-\mathrm{K}^{\mathrm{b}} \mathrm{\alpha} 3 \mathrm{~A} 2$
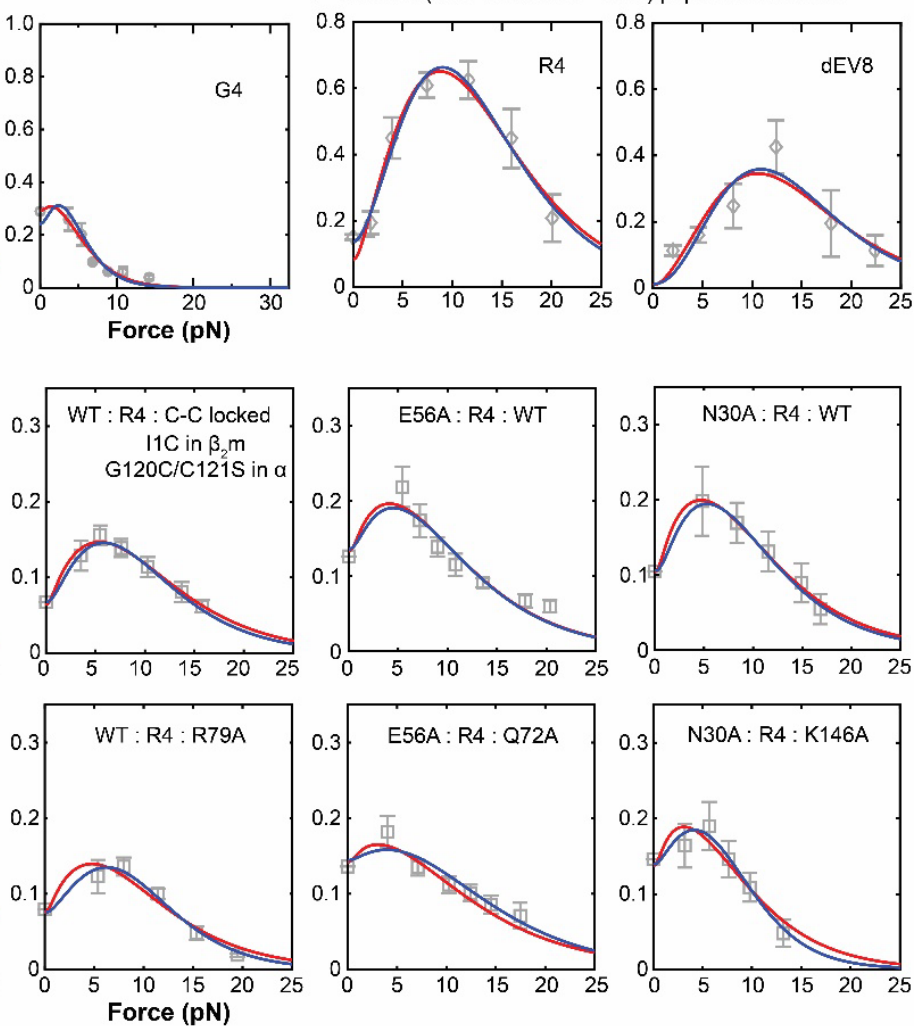

E TCRs:NP ${ }_{366}: \mathrm{H}^{2}-\mathrm{D}^{\mathrm{b}(\mathrm{D} 227 \mathrm{~K})}$
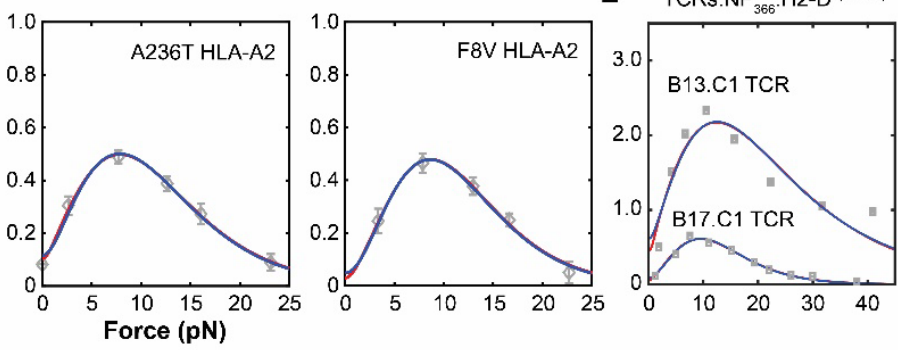
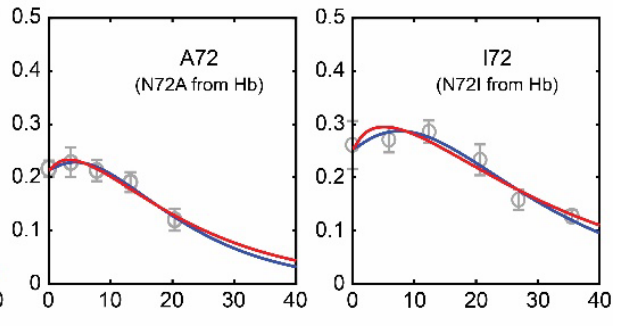

I E8 TCR:TPI:HLA-DR1
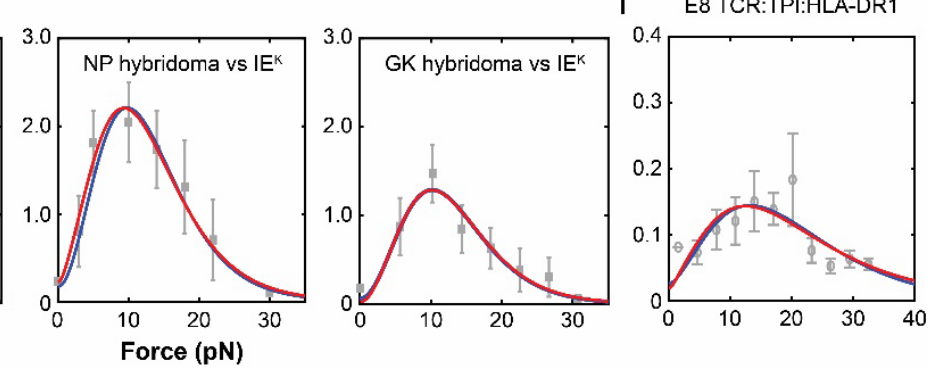
Fig. S8. Comparing fitting curves of class I and class II models for the same data. Fitting of theoretical $1 / k(F)$ curves predicted by the class I (blue) or class II (red) model to experimental lifetime vs force data of TCR bonds with pMHC-I (A-F) or pMHC-II (G-I) ligands, as described below (partly presented in Fig. S1, re-analyzed from (2, 3, 5, 9, 26, 52)): OT1 (A) or 2C (B) TCR expressed on $\mathrm{CD}^{+}$naïve $\mathrm{T}$ cells interacting with indicated p:H2$\mathrm{K}^{\mathrm{b}} \alpha 3 \mathrm{~A} 2$; WT or mutant $2 \mathrm{C}$ (C) or 1G4 (D) TCR expressed on hybridomas interacting with indicated peptides presented by WT or mutant $\mathrm{H} 2-\mathrm{K}^{\mathrm{b}}$ or HLA-A2. B13.C1 and B17.C1 TCR expressed on hybridomas interacted with $\mathrm{NP}_{366}$ bound to the $\mathrm{D} 227 \mathrm{~K}$ mutant of $\mathrm{H}-2 \mathrm{D}^{\mathrm{b}}$ to prevent CD8 binding (E). Soluble N15 TCR $\alpha \beta$ interacting with indicated p:H2-K ${ }^{\mathrm{b}}(\mathrm{F})$. 3.L2 TCR expressed on $\mathrm{CD} 4{ }^{-} \mathrm{CD} 8^{+} \mathrm{T}$ cells interacting with indicated $\mathrm{p}: \mathrm{I}_{-} \mathrm{E}^{\mathrm{k}}(\mathrm{G})$. WT or mutant $2 \mathrm{~B} 4$ TCRs expressed on CD4 ${ }^{-}$hybridomas interacting with $\mathrm{K} 5 \mathrm{I}^{-\mathrm{E}^{\mathrm{k}}}(\mathrm{H})$. E8 TCR expressed on CD4Jurkat cells interacting with TPI:HLA-DR1 (I). See Tables S3 and S5 for lists of the interacting molecules. 

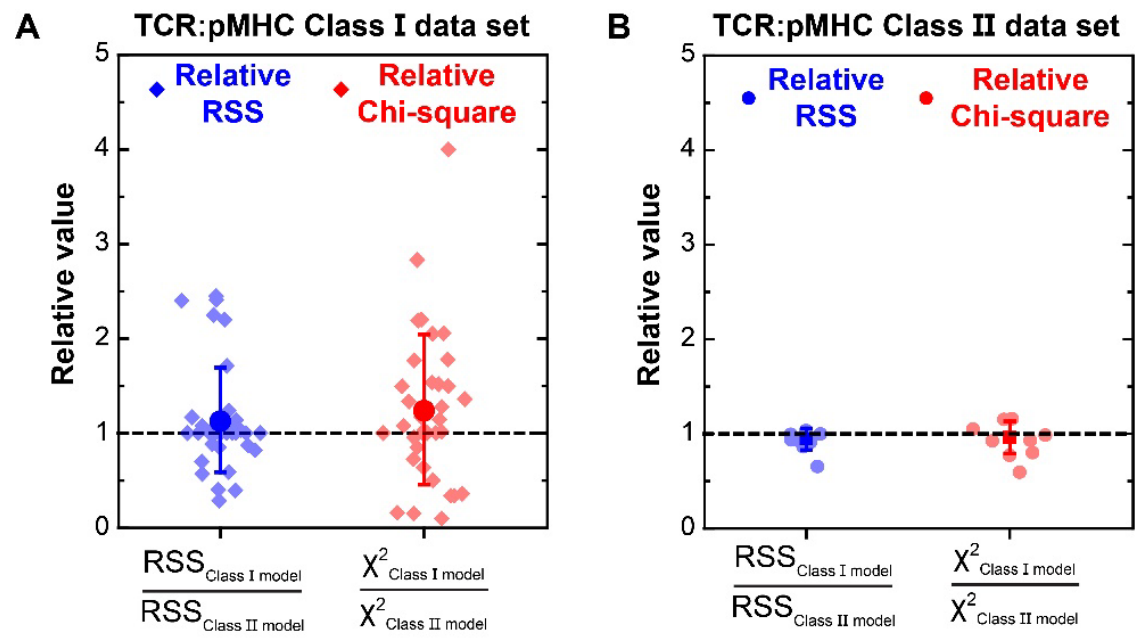

Fig. S9. Relative goodness-of-fit of class I and II models to class I and class II data. Ratios of residual sum of squares (RSS, blue) and Chi-square $\left(\chi^{2}\right.$, red) values obtained using class I model over those using class II model to fit pMHC-I (A) or pMHC-II (B) data. Each diamond represents an individual relative value evaluated from on bond lifetime vs force curve from Fig. S8. Circles and error bars $=$ mean \pm s.d. 

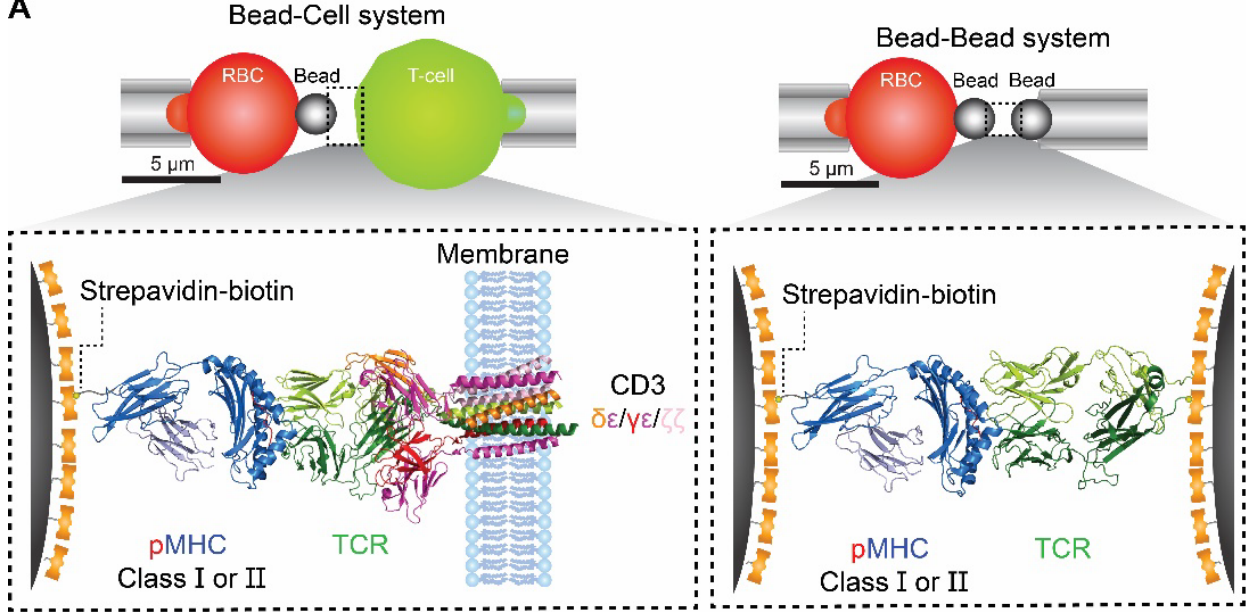

B
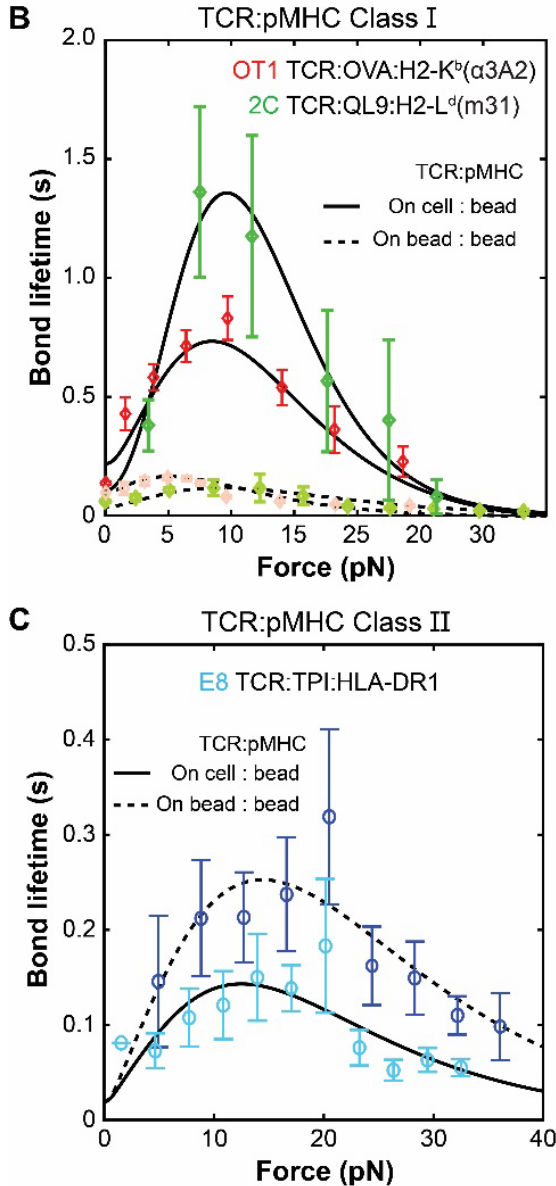

D

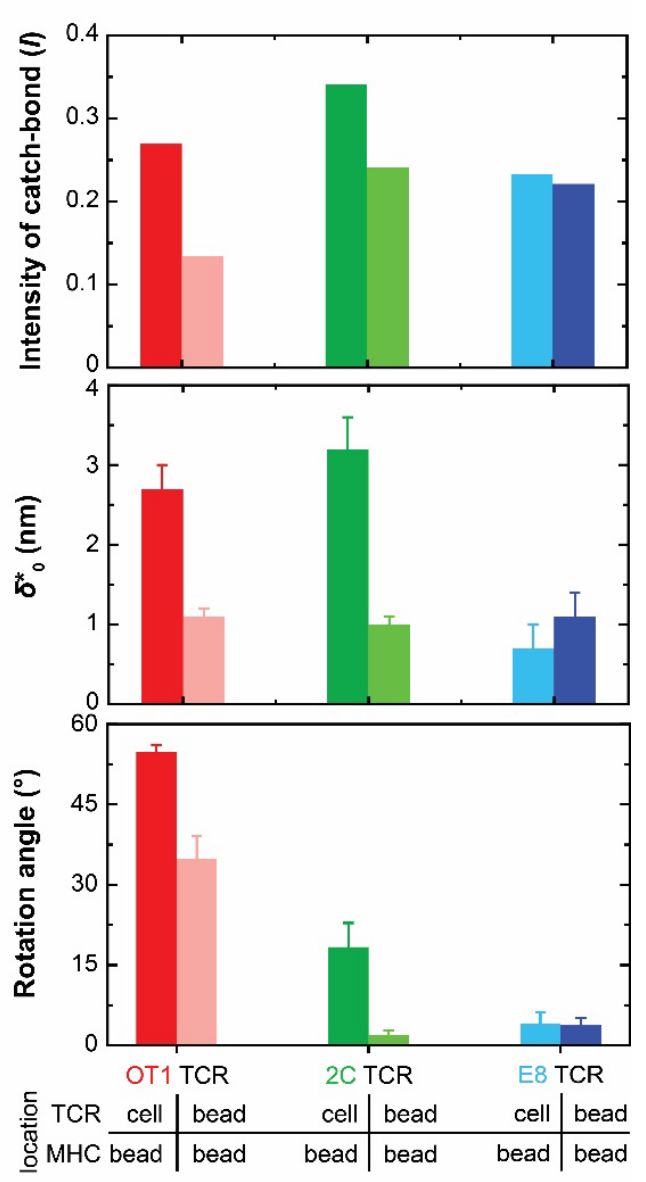

Fig. S10. Comparison of pMHC interactions with cell surface and purified TCRs. (A)

Schematics of two biomembrane force probe (BFP) experiments. The pMHC is coated on BFP beads (left on each panel) and the TCR-CD3 complex (right, middle panel) is expressed on cell surface (upper on left panel) or the purified TCR $\alpha \beta$ (right, right panel) is coated on a glass bead (lower on left panel). The TCR-pMHC complexes in the middle and right panels are drawn based on cryoEM (6JXR for TCR-CD3 complex) or crystal (2CKB for TCR-pMHC 
complex) structures. (B, C) Fitting of theoretical $1 / k(F)$ (curves) to experimental bond lifetime vs force data (points, mean \pm sem) of the following interactions: OT1 or 2C TCR respectively expressed on $\mathrm{CD}^{+}$naïve T cells (red) or CD8- hybridomas (green) or coated on beads (pink for OT1 and yellow-green for $2 \mathrm{C}$ ) respectively interacting with OVA:H2-K ${ }^{\mathrm{b}} \alpha 3 \mathrm{~A} 2$ or QL9:H2$\mathrm{L}^{\mathrm{d}}(\mathrm{m} 3)$, both MHC class I molecules (51) (B) or of E8 TCR expressed on CD8- Jurkat (skyblue) or coated on beads (blue) interacting with TPI:HLA-DR1, a MHC class II (52) (C). (D) Comparison of the catch bond intensity (top) and best-fit parameters $\boldsymbol{\delta}_{\mathbf{0}}^{*}$ (middle) and $\boldsymbol{\theta}$ (bottom) evaluated from data of pMHC interactions with cell surface vs purified TCRs in B and C. See Table S3 and S5 for lists of the interacting molecules. 
Table S1. Summary of Model Constants.

\begin{tabular}{|c|c|c|c|}
\hline Parameters & Symbol & Value & Reference \\
\hline Elastic modulus of the folded globular domain & $E_{\mathrm{d}}$ & $100 \mathrm{pN}$ & $(56)$ \\
\hline Average contour length & $l_{\mathrm{c}}$ & $0.36 \mathrm{~nm}$ & $(29,57,58)$ \\
\hline Persistence length & $l_{\mathrm{p}}$ & $0.39 \mathrm{~nm}$ & $(29,57,58)$ \\
\hline Elastic modulus of polypeptides & $E_{\mathrm{p}}$ & $50 \mu \mathrm{N}$ & $(57)$ \\
\hline Force-free extension of bound state & $d_{\mathrm{N}, \mathrm{c}}$ & $10.9-12.7 \mathrm{~nm}$ & ${ }^{\ddagger}$ \\
\hline Force-free length of TCR $\alpha \beta$ constant domain & $d_{\mathrm{C} \alpha \beta, \mathrm{c}}$ & $3.3-3.8 \mathrm{~nm}$ & ${ }^{\ddagger}$ \\
\hline Angle shown in Fig. 1B & $\varphi$ & $5-23.5^{\circ}$ & ${ }^{\ddagger}$ \\
\hline
\end{tabular}

${ }^{\ddagger}$ Detailed values for each of the OT1, 2C, 1G4, N15, B13.C1, B17.C1, B17.R1, B17.R2, 3.L2, E8, and 2B4 TCR structures are described in Supplemental Model Derivations. 
Table S2. Finding the Best-fit Parameters for OT1 TCR-OVA:H2-K ${ }^{\mathrm{b}} \mathbf{a}$ A2 Bond.

\begin{tabular}{|ccccccc|}
\hline $\boldsymbol{n}^{*}$ & $\boldsymbol{d}_{\boldsymbol{\alpha} \mathbf{3}}(\mathrm{nm})^{\ddagger}$ & $\boldsymbol{\theta}\left(^{\circ}\right)^{\ddagger}$ & $\boldsymbol{n}^{*} \boldsymbol{l}_{\mathbf{c}}(\mathrm{nm})$ & $\boldsymbol{\delta}_{\mathbf{l}}(\mathrm{nm})$ & $\boldsymbol{\delta}_{\mathbf{0}}(\mathrm{nm})^{\ddagger}$ & $\boldsymbol{k}_{\mathbf{0}}\left(\mathrm{s}^{-1}\right)^{\ddagger}$ \\
\hline 1 & 5.2 & 61.9 & 0.36 & -0.8 & 0.7 & 4.84 \\
2 & 5.1 & 59.7 & 0.72 & -0.5 & 1.1 & 4.66 \\
3 & 4.9 & 57.3 & 1.08 & 0.0 & 1.4 & 4.54 \\
4 & 4.7 & 55.4 & 1.44 & 0.2 & 1.7 & 4.44 \\
5 & 4.5 & 54.1 & 1.80 & 0.6 & 1.9 & 4.34 \\
6 & 4.4 & 53.5 & 2.16 & 1.0 & 2.1 & 4.26 \\
7 & 4.3 & 53.4 & 2.52 & 1.3 & 2.2 & 4.20 \\
8 & 4.2 & 53.6 & 2.88 & 1.7 & 2.4 & 4.14 \\
9 & 4.1 & 54.1 & 3.24 & 2.0 & 2.5 & 4.09 \\
10 & 4.1 & 54.8 & 3.60 & 2.4 & 2.6 & 4.04 \\
$\mathbf{1 1}$ & $\mathbf{4 . 0}$ & $\mathbf{5 5 . 5}$ & $\mathbf{3 . 9 6}$ & $\mathbf{2 . 7}$ & $\mathbf{2 . 7}$ & $\mathbf{4 . 0 0}$ \\
12 & 4.0 & 56.4 & 4.32 & 3.1 & 2.8 & 3.73 \\
13 & 1.8 & 25.8 & 4.68 & 3.5 & 3.9 & 3.76 \\
14 & 2.4 & 70.5 & 5.04 & 3.9 & 3.3 & 3.87 \\
\hline
\end{tabular}

'The fitting parameters derived from model (see Supplementary Information text, Section A.3). 
Table S3. Best-fitting Parameters for the TCR-pMHC-I Bonds

\begin{tabular}{|c|c|c|c|c|c|c|c|c|}
\hline TCR & peptide & $\begin{array}{c}\text { MHC } \\
\text { class I }\end{array}$ & $\boldsymbol{n}^{*}$ & $\begin{array}{c}\boldsymbol{d}_{\alpha 3} \pm \text { error } \\
(\mathrm{nm})\end{array}$ & $\begin{array}{c}\boldsymbol{\theta} \pm \text { error } \\
\left({ }^{\circ}\right)\end{array}$ & $\begin{array}{c}\boldsymbol{\delta}_{\mathbf{0}} \pm \text { error } \\
(\mathrm{nm})\end{array}$ & $\begin{array}{c}\boldsymbol{k}_{\mathbf{0}} \pm \text { error } \\
\left(\mathrm{s}^{-1}\right)\end{array}$ & $\begin{array}{c}\Delta G_{0}^{\ddagger} \pm \text { error } \\
\left(k_{\mathrm{B}} T\right)\end{array}$ \\
\hline $\mathrm{OT}^{\dagger}{ }^{\dagger}$ & OVA & $\begin{array}{l}\mathrm{H} 2-\mathrm{K}^{\mathrm{b}} \\
(\alpha 3 \mathrm{~A} 2)\end{array}$ & 11 & $4.0 \pm 0.2$ & $55.5 \pm 1.5$ & $2.7 \pm 0.3$ & $4.0 \pm 2.3$ & $12.4 \pm 0.5$ \\
\hline OT1P & OVA & $\begin{array}{l}\mathrm{H} 2-\mathrm{K}^{\mathrm{b}} \\
(\alpha 3 \mathrm{~A} 2)\end{array}$ & 6 & $4.0 \pm 0.2$ & $34.9 \pm 4.3$ & $1.1 \pm 0.1$ & $9.6 \pm 1.6$ & $11.6 \pm 0.1$ \\
\hline $\mathrm{OT}^{\dagger}{ }^{\dagger}$ & $\mathrm{A} 2$ & $\begin{array}{l}\mathrm{H} 2-\mathrm{K}^{\mathrm{b}} \\
(\alpha 3 \mathrm{~A} 2)\end{array}$ & 7 & $3.9 \pm 0.2$ & $44.0 \pm 3.1$ & $1.1 \pm 0.1$ & $4.1 \pm 1.0$ & $12.4 \pm 0.2$ \\
\hline OT1 $^{\dagger}$ & G4 & $\begin{array}{l}\mathrm{H} 2-\mathrm{K}^{\mathrm{b}} \\
(\alpha 3 \mathrm{~A} 2)\end{array}$ & 9 & $5.0 \pm 0.4$ & $35.2 \pm 2.2$ & $2.7 \pm 0.4$ & $4.2 \pm 1.1$ & $12.4 \pm 0.2$ \\
\hline $\mathrm{OT}^{\dagger}{ }^{\dagger}$ & E1 & $\begin{array}{l}\mathrm{H} 2-\mathrm{K}^{\mathrm{b}} \\
(\alpha 3 \mathrm{~A} 2)\end{array}$ & 0 & $0.0 \pm 0.0$ & $1.0 \pm 1.2$ & $0.9 \pm 0.1$ & $2.4 \pm 0.2$ & $13.0 \pm 0.1$ \\
\hline $\mathrm{OT}^{\dagger}{ }^{\dagger}$ & R4 & $\begin{array}{l}\mathrm{H} 2-\mathrm{K}^{\mathrm{b}} \\
(\alpha 3 \mathrm{~A} 2)\end{array}$ & 0 & $0.0 \pm 0.0$ & $0.0 \pm 0.9$ & $1.0 \pm 0.3$ & $2.0 \pm 0.3$ & $13.1 \pm 0.1$ \\
\hline OT1* & OVA & $\begin{array}{l}\mathrm{H} 2-\mathrm{K}^{\mathrm{b}} \\
(\alpha 3 \mathrm{~A} 2)\end{array}$ & 6 & $1.6 \pm 0.2$ & $17.5 \pm 2.9$ & $1.6 \pm 0.2$ & $3.3 \pm 0.6$ & $12.6 \pm 0.2$ \\
\hline OT1* & OVA & $\mathrm{H} 2-\mathrm{K}^{\mathrm{b}}$ & 11 & $1.9 \pm 0.3$ & $26.6 \pm 4.9$ & $3.1 \pm 0.3$ & $3.6 \pm 0.3$ & $12.5 \pm 0.1$ \\
\hline OT1* & Q4H7 & $\begin{array}{l}\mathrm{H} 2-\mathrm{K}^{\mathrm{b}} \\
(\alpha 3 \mathrm{~A} 2)\end{array}$ & 1 & $0.0 \pm 0.0$ & $0.0 \pm 0.2$ & $0.4 \pm 0.2$ & $4.3 \pm 0.8$ & $12.4 \pm 0.2$ \\
\hline OT1* & Q4R7 & $\begin{array}{l}\mathrm{H} 2-\mathrm{K}^{\mathrm{b}} \\
(\alpha 3 \mathrm{~A} 2)\end{array}$ & 1 & $0.0 \pm 0.0$ & $0.0 \pm 0.2$ & $0.4 \pm 0.1$ & $3.8 \pm 0.8$ & $12.4 \pm 0.2$ \\
\hline $2 \mathrm{C}^{\dagger}$ & R4 & $\begin{array}{l}\mathrm{H} 2-\mathrm{K}^{\mathrm{b}} \\
(\alpha 3 \mathrm{~A} 2)\end{array}$ & 7 & $2.5 \pm 0.5$ & $19.3 \pm 1.3$ & $2.3 \pm 0.2$ & $7.4 \pm 4.8$ & $11.8 \pm 0.5$ \\
\hline $2 \mathrm{C}^{\dagger}$ & dEV8 & $\begin{array}{l}\mathrm{H} 2-\mathrm{K}^{\mathrm{b}} \\
(\alpha 3 \mathrm{~A} 2)\end{array}$ & 3 & $2.6 \pm 0.5$ & $13.3 \pm 1.7$ & $2.0 \pm 0.2$ & $75.6 \pm 18.7$ & $9.5 \pm 0.2$ \\
\hline $2 \mathrm{C}^{\dagger}$ & L4 & $\begin{array}{l}\mathrm{H} 2-\mathrm{K}^{\mathrm{b}} \\
(\alpha 3 \mathrm{~A} 2)\end{array}$ & 0 & $0.0 \pm 0.0$ & $0.0 \pm 0.2$ & $0.4 \pm 0.1$ & $3.8 \pm 1.5$ & $12.5 \pm 0.3$ \\
\hline $2 C^{*}$ & R4 & $\begin{array}{l}\mathrm{H} 2-\mathrm{K}^{\mathrm{b}} \\
(\alpha 3 \mathrm{~A} 2) \\
\end{array}$ & 6 & .1 & $7.3 \pm 1.6$ & 0.2 & 0.3 & $12.5 \pm 0.1$ \\
\hline $2 C^{*}$ & R4 & $\mathrm{H} 2-\mathrm{K}^{\mathrm{b}}$ & 12 & $1.7 \pm 0.3$ & $21.8 \pm 2.5$ & $2.8 \pm 0.3$ & $5.5 \pm 0.6$ & $12.1 \pm 0.1$ \\
\hline $2 \mathrm{C}$ & R4 & $\mathrm{H} 2-\mathrm{K}^{\mathrm{b}}$ & 7 & $4.0 \pm 0.3$ & $28.2 \pm 2.0$ & $1.5 \pm 0.4$ & $8.9 \pm 4.7$ & $11.6 \pm 0.4$ \\
\hline $2 \mathrm{C}$ & R4 & $\begin{array}{c}\mathrm{H} 2-\mathrm{K}^{\mathrm{b}} \\
\mathrm{C}-\mathrm{C} \\
\text { locked }^{*}\end{array}$ & 7 & $4.1 \pm 0.2$ & $24.9 \pm 2.0$ & $1.6 \pm 0.3$ & $14.8 \pm 4.4$ & $11.1 \pm 0.3$ \\
\hline $2 \mathrm{C}$ & EVSV & $\mathrm{H} 2-\mathrm{K}^{\mathrm{b}}$ & 2 & $0.4 \pm 0.2$ & $6.4 \pm 1.8$ & $0.9 \pm 0.3$ & $9.0 \pm 2.8$ & $11.6 \pm 0.3$ \\
\hline $2 \mathrm{C}$ & L4 & $\mathrm{H} 2-\mathrm{K}^{\mathrm{b}}$ & 1 & $0.1 \pm 0.1$ & $2.1 \pm 1.5$ & $0.6 \pm 0.1$ & $6.8 \pm 1.3$ & $11.9 \pm 0.1$ \\
\hline $\begin{array}{c}2 \mathrm{C} \\
\mathrm{E} 56 \mathrm{~A}\end{array}$ & R4 & $\mathrm{H} 2-\mathrm{K}^{\mathrm{b}}$ & 9 & $4.1 \pm 0.5$ & $20.7 \pm 3.3$ & $1.2 \pm 0.2$ & $7.4 \pm 3.5$ & $11.8 \pm 0.4$ \\
\hline $\begin{array}{c}2 \mathrm{C} \\
\mathrm{N} 30 \mathrm{~A}\end{array}$ & R4 & $\mathrm{H} 2-\mathrm{K}^{\mathrm{b}}$ & 7 & $4.3 \pm 0.1$ & $23.6 \pm 2.8$ & $1.4 \pm 0.4$ & $9.5 \pm 3.6$ & $11.6 \pm 0.3$ \\
\hline $2 \mathrm{C}$ & R4 & $\begin{array}{l}\mathrm{H} 2-\mathrm{K}^{\mathrm{b}} \\
\mathrm{Q} 72 \mathrm{~A}\end{array}$ & 7 & $4.2 \pm 0.4$ & $25.3 \pm 2.3$ & $1.3 \pm 0.3$ & $8.0 \pm 4.5$ & $11.7 \pm 0.4$ \\
\hline $2 \mathrm{C}$ & R4 & $\begin{array}{l}\mathrm{H} 2-\mathrm{K}^{\mathrm{b}} \\
\mathrm{K} 146 \mathrm{~A} \\
\end{array}$ & 4 & $0.4 \pm 0.2$ & $4.7 \pm 1.0$ & $1.1 \pm 0.2$ & $6.7 \pm 1.3$ & $11.9 \pm 0.1$ \\
\hline $2 \mathrm{C}$ & R4 & $\begin{array}{l}\mathrm{H} 2-\mathrm{K}^{\mathrm{b}} \\
\mathrm{R} 79 \mathrm{~A} \\
\end{array}$ & 4 & 0.6 & .5 & 0.6 & 6.3 & 0.4 \\
\hline $\begin{array}{c}2 \mathrm{C} \\
\mathrm{E} 56 \mathrm{~A}\end{array}$ & R4 & $\begin{array}{l}\mathrm{H} 2-\mathrm{K}^{\mathrm{b}} \\
\mathrm{Q} 72 \mathrm{~A} \\
\end{array}$ & 4 & $0.4 \pm 0.3$ & 1.2 & $1.1 \pm 0.3$ & $6.9 \pm 2.7$ & $11.9 \pm 0.3$ \\
\hline $\begin{array}{l}2 \mathrm{C} \\
\mathrm{N} 30 \mathrm{~A}\end{array}$ & R4 & $\begin{array}{l}\mathrm{H} 2-\mathrm{K}^{\mathrm{b}} \\
\mathrm{K} 146 \mathrm{~A}\end{array}$ & 2 & $0.4 \pm 0.2$ & $6.8 \pm 0.9$ & $1.5 \pm 0.4$ & $7.2 \pm 3.8$ & $11.8 \pm 0.4$ \\
\hline $2 \mathrm{C}^{\dagger}$ & QL9 & $\begin{array}{l}\mathrm{H} 2-\mathrm{L}^{\mathrm{d}} \\
(\mathrm{m} 31)\end{array}$ & 6 & $2.5 \pm 0.7$ & $18.4 \pm 4.5$ & $3.2 \pm 0.4$ & $9.5 \pm 7.9$ & $11.6 \pm 0.6$ \\
\hline $2 C^{\mathbb{P}}$ & QL9 & $\begin{array}{l}\mathrm{H} 2-\mathrm{L}^{\mathrm{d}} \\
\text { (m31) }\end{array}$ & 3 & $2.0 \pm 0.6$ & $2.0 \pm 0.9$ & $1.0 \pm 0.1$ & $26.9 \pm 7.0$ & $10.5 \pm 0.2$ \\
\hline
\end{tabular}




\begin{tabular}{|c|c|c|c|c|c|c|c|c|}
\hline $1 G 4$ & $9 \mathrm{C}$ & $\mathrm{HLA}-\mathrm{A} 2$ & 4 & $1.8 \pm 0.7$ & $10.1 \pm 0.5$ & $2.7 \pm 0.4$ & $13.7 \pm 3.5$ & $11.2 \pm 0.2$ \\
\hline $1 \mathrm{G} 4$ & $9 \mathrm{C}$ & $\begin{array}{c}\mathrm{HLA}-\mathrm{A} 2 \\
\mathrm{R} 75 \mathrm{~A}\end{array}$ & 6 & $1.4 \pm 0.2$ & $9.4 \pm 0.4$ & $3.7 \pm 0.3$ & $8.1 \pm 8.0$ & $11.0 \pm 0.7$ \\
\hline $1 \mathrm{G} 4$ & $9 \mathrm{C}$ & $\begin{array}{c}\mathrm{HLA}-\mathrm{A} 2 \\
\mathrm{~A} 236 \mathrm{~T}\end{array}$ & 5 & $4.2 \pm 0.2$ & $29.4 \pm 2.6$ & $1.8 \pm 0.3$ & $8.6 \pm 2.0$ & $11.7 \pm 0.2$ \\
\hline $1 \mathrm{G} 4$ & $9 \mathrm{C}$ & $\begin{array}{c}\mathrm{HLA}-\mathrm{A} 2 \\
\mathrm{~F} 8 \mathrm{~V}\end{array}$ & 4 & $4.1 \pm 0.4$ & $26.8 \pm 1.4$ & $2.0 \pm 0.4$ & $19.7 \pm 4.5$ & $10.8 \pm 0.2$ \\
\hline $\mathrm{N} 15^{\pi}$ & $\mathrm{VSV} 8$ & $\mathrm{H} 2-\mathrm{K}^{\mathrm{b}}$ & 9 & $3.2 \pm 0.3$ & $32.0 \pm 4.6$ & $4.5 \pm 0.5$ & $32.9 \pm 10.0$ & $10.3 \pm 0.2$ \\
\hline $\mathrm{N} 15^{\pi}$ & $\mathrm{L} 4$ & $\mathrm{H} 2-\mathrm{K}^{\mathrm{b}}$ & 5 & $1.8 \pm 0.3$ & $13.7 \pm 1.5$ & $4.3 \pm 0.5$ & $48.1 \pm 21.1$ & $9.9 \pm 0.4$ \\
\hline $\mathrm{N} 15^{\pi}$ & $\mathrm{SEV} 9$ & $\mathrm{H} 2-\mathrm{K}^{\mathrm{b}}$ & 0 & $0.0 \pm 0.0$ & $0.0 \pm 0.0$ & $0.6 \pm 0.1$ & $0.6 \pm 0.1$ & $14.3 \pm 0.2$ \\
\hline $\begin{array}{c}\mathrm{B} 13 . \\
\mathrm{C} 1^{\#}\end{array}$ & $\mathrm{NP}_{366}$ & $\begin{array}{c}\mathrm{H}-2 \mathrm{D}^{\mathrm{b}} \\
(\mathrm{D} 227 \mathrm{~K})\end{array}$ & 8 & $4.2 \pm 0.4$ & $40.0 \pm 6.2$ & $1.4 \pm 0.3$ & $1.6 \pm 0.8$ & $13.3 \pm 0.4$ \\
\hline $\begin{array}{c}\mathrm{B} 17 . \\
\mathrm{C} 1^{\#}\end{array}$ & $\mathrm{NP}_{366}$ & $\begin{array}{c}\mathrm{H}-2 \mathrm{D}^{\mathrm{b}} \\
(\mathrm{D} 227 \mathrm{~K})\end{array}$ & 6 & $4.2 \pm 00.5$ & $33.2 \pm 4.1$ & $2.2 \pm 0.5$ & $11.3 \pm 6.6$ & $11.4 \pm 0.2$ \\
\hline $\begin{array}{c}\mathrm{B} 17 . \\
\mathrm{R} 1^{\#}\end{array}$ & $\mathrm{NP}_{366}$ & $\begin{array}{c}\mathrm{H}-2 \mathrm{D}^{\mathrm{b}} \\
(\mathrm{D} 227 \mathrm{~K})\end{array}$ & 0 & $0.0 \pm 0.0$ & $0.0 \pm 0.0$ & $0.6 \pm 0.2$ & $0.3 \pm 0.1$ & $14.9 \pm 0.3$ \\
\hline $\begin{array}{c}\mathrm{B} 17 . \\
\mathrm{R} 2^{\#}\end{array}$ & $\mathrm{NP}_{366}$ & $\begin{array}{c}\mathrm{H}-2 \mathrm{D}^{\mathrm{b}} \\
(\mathrm{D} 227 \mathrm{~K})\end{array}$ & 3 & $0.4 \pm 0.3$ & $0.4 \pm 0.2$ & $1.2 \pm 0.2$ & $0.4 \pm 0.1$ & $14.9 \pm 0.2$ \\
\hline
\end{tabular}

PPurified recombinant TCRs coated on beads were used in BFP measurement.

"OT1 or $2 \mathrm{C}$ TCR expressed on $\mathrm{CD} 4^{+} \mathrm{CD} 8^{+}$thymocytes were used.

${ }^{\dagger} \mathrm{OT} 1$ or $2 \mathrm{C}$ TCR expressed on naïve T cells from transgenic mice were used. In other cases, $2 \mathrm{C}$ or 1G4 TCR expressed on $58 \alpha^{-1} \beta^{-1}$ hybridomas and J76 Jurkat cells were used.

ISoluble mouse N15 TCR $\alpha \beta$ was used to measure bond lifetime in optical tweezers (4).

"Mouse TRBV TCRs (B13.C1 and B17.C1 with canonical docking orientation and B17.R1 and B17.R2 with reverse docking orientation) expressed on $5 \mathrm{KC}$ hybridomas interacted with $\mathrm{NP}_{366}$ bound to the $\mathrm{D} 227 \mathrm{~K}$ mutant of $\mathrm{H}-2 \mathrm{D}^{\mathrm{b}}$ to prevent $\mathrm{CD} 8$ binding (11)

"I1C mutation in $\beta 2 \mathrm{~m}$ domain, G120C and C121S mutations in $\alpha$ domain $\left(\mathrm{H} 2-\mathrm{K}^{\mathrm{b}}\right)$ were introduced. Note that the best-fit parameters were chosen by the procedure shown in Table S2 or determined as the parameter set with the closest $\delta_{1}$ value to $\delta_{0}^{*}$ as well as the smallest RSS. All errors are standard error of mean after optimizing the best-fit parameters. All values were rounded to one decimal place. 
Table S4. Finding the Best-fit Parameters for the 3.L2 TCR-Hb:I-E ${ }^{\mathrm{k}}$ Bond

\begin{tabular}{|ccccccc|}
\hline $\boldsymbol{n}_{\text {MHC }}^{*}$ & $\boldsymbol{d}_{\text {B.I }}(\mathrm{nm})^{\ddagger}$ & $\boldsymbol{\theta}\left({ }^{\circ}\right)^{\ddagger}$ & $\boldsymbol{n}^{*} \boldsymbol{l}_{\mathbf{c}}(\mathrm{nm})$ & $\begin{array}{c}\boldsymbol{\delta}_{\mathbf{I}} \\
(\mathrm{nm})\end{array}$ & $\begin{array}{c}\boldsymbol{\delta}_{\mathbf{0}} \\
(\mathrm{nm})^{\ddagger}\end{array}$ & $\begin{array}{c}\boldsymbol{k}_{\mathbf{0}} \\
\left(\mathrm{s}^{-1}\right)^{\ddagger}\end{array}$ \\
\hline 1 & 8.9 & 0.0 & 0.4 & 0.6 & 0.7 & 2.0 \\
2 & 8.8 & 0.0 & 0.7 & 0.9 & 1.2 & 1.9 \\
3 & 8.6 & 0.5 & 1.1 & 1.3 & 1.5 & 1.8 \\
$\mathbf{4}$ & $\mathbf{7 . 9}$ & $\mathbf{3 . 1}$ & $\mathbf{1 . 4}$ & $\mathbf{1 . 6}$ & $\mathbf{1 . 6}$ & $\mathbf{1 . 8}$ \\
5 & 7.9 & 2.6 & 1.8 & 2.0 & 1.7 & 1.7 \\
6 & 7.8 & 2.1 & 2.2 & 2.4 & 1.9 & 1.7 \\
7 & 8.0 & 1.5 & 2.5 & 2.7 & 2.1 & 1.6 \\
8 & 7.8 & 0.6 & 2.9 & 3.1 & 2.2 & 1.6 \\
\hline
\end{tabular}

${ }^{\dagger} n^{*}$ can be estimated from $n_{\mathrm{pMHC}}^{*}$.

${ }^{\ddagger}$ See Supplementary Model Derivations, Section B. 
Table S5. Best-fit parameters for TCR-pMHC-II Bonds

\begin{tabular}{|c|c|c|c|c|c|c|c|c|}
\hline TCR & peptide & $\begin{array}{c}\text { MHC } \\
\text { class II }\end{array}$ & $\boldsymbol{n}^{*}$ & $\begin{array}{c}\boldsymbol{d}_{\text {B.I }} \pm \text { error } \\
(\mathrm{nm})\end{array}$ & $\begin{array}{c}\boldsymbol{\theta} \pm \text { error } \\
\left({ }^{\circ}\right)\end{array}$ & $\begin{array}{c}\boldsymbol{\delta}_{0} \pm \text { error } \\
(\mathrm{nm})\end{array}$ & $\begin{array}{c}\boldsymbol{k}_{0} \pm \text { error } \\
\left(\mathrm{s}^{-1}\right)\end{array}$ & $\begin{array}{c}\Delta G_{0}^{\ddagger} \pm \text { error } \\
\left(k_{\mathrm{B}} \mathrm{T}\right)\end{array}$ \\
\hline 3.L2 & $\mathrm{Hb}$ & I-E & 4 & $8.0 \pm 0.6$ & $3.2 \pm 0.6$ & $1.6 \pm 0.5$ & $1.8 \pm 0.3$ & $13.2 \pm 0.2$ \\
\hline 3.L2 & T72 & $I-E^{k}$ & 3 & $8.7 \pm 0.1$ & $0.1 \pm 0.1$ & $1.2 \pm 0.3$ & $2.6 \pm 0.6$ & $12.9 \pm 0.3$ \\
\hline 3.L2 & A72 & $I-E^{k}$ & 2 & $9.3 \pm 0.2$ & $0.0 \pm 0.3$ & $0.2 \pm 0.0$ & $4.7 \pm 0.5$ & $12.3 \pm 0.1$ \\
\hline 3.L2 & 172 & $I-E^{k}$ & 1 & $9.1 \pm 0.1$ & $0.0 \pm 0.3$ & $0.1 \pm 0.0$ & $3.9 \pm 0.6$ & $12.4 \pm 0.2$ \\
\hline E8 & TPI & $\begin{array}{l}\text { HLA- } \\
\text { DR1 }\end{array}$ & 2 & $8.6 \pm 0.1$ & $4.1 \pm 0.4$ & $0.7 \pm 0.3$ & $78.3 \pm 2.7$ & $9.5 \pm 0.1$ \\
\hline E8P & TPI & $\begin{array}{l}\text { HLA- } \\
\text { DR1 }\end{array}$ & 3 & $8.5 \pm 0.1$ & $4.0 \pm 0.5$ & $1.1 \pm 0.3$ & $41.4 \pm 9.4$ & $10.1 \pm 0.2$ \\
\hline $\begin{array}{l}\text { 2B4 } \\
\text { (NP) }\end{array}$ & K5 & $\mathrm{I}-\mathrm{E}^{\mathrm{k}}$ & 6 & $7.9 \pm 0.3$ & $7.3 \pm 1.3$ & $2.0 \pm 0.2$ & $4.5 \pm 2.4$ & $12.3 \pm 0.4$ \\
\hline $\begin{array}{c}\text { 2B4 } \\
\text { (WT) }\end{array}$ & K5 & $I-E^{k}$ & 4 & $8.8 \pm 0.3$ & $2.3 \pm 0.7$ & $1.8 \pm 0.4$ & $64.0 \pm 9.1$ & $9.7 \pm 0.1$ \\
\hline $\begin{array}{l}\text { 2B4 } \\
\text { (GK) }\end{array}$ & K5 & $I-E^{k}$ & 3 & $8.9 \pm 0.2$ & $1.9 \pm 1.2$ & $1.4 \pm 0.5$ & $40.1 \pm 9.8$ & $10.1 \pm 0.2$ \\
\hline
\end{tabular}

PThe recombinant TCR coated to the bead was used in BFP measurement.

Note that the best-fit parameters were chosen using the procedure shown in Table S4 or determined as the parameter set with the $\delta_{\mathrm{z}}\left(n^{*}\right)$ value closest to $\delta_{0}^{*}$ as well as the smallest RSS. All errors are standard error of mean after optimizing the best-fit parameters. All values were rounded to one decimal place. 